Elsevier required licence: (c) $<2016\rangle$. This manuscript version is made available under the CC-BY-NC-ND 4.0 license http://creativecommons.org/licenses/bync-nd/4.0/ 


\title{
Microfoundations for Switching Behavior in Heterogeneous Agent Models: An Experiment*
}

\author{
Mikhail Anufriev ${ }^{\mathrm{a}, \uparrow} \quad$ Te Bao ${ }^{\mathrm{b}, \ddagger} \quad$ Jan Tuinstra ${ }^{\mathrm{c}, \S}$
}

\begin{abstract}
We run a laboratory experiment to study how human subjects switch between several profitable alternatives, framed as mutual funds, in order to provide a microfoundation for so-called heterogeneous agent models. The participants in our experiment have to choose repeatedly between two, three or four experimental funds. The time series of fund returns are exogenously generated prior to the experiment and participants are paid for each period according to the return of the fund they choose. For most cases participants' decisions can be successfully described by a discrete choice switching model, often applied in heterogeneous agent models, provided that a predisposition towards one of the funds is included. The estimated intensity of choice parameter of the discrete choice model depends on the structure of the fund returns. In particular, it increases with correlation between past and future returns. This suggests human subjects do not myopically chase past returns, but are more likely to do so when past returns are more predictive of future returns, a feature that is absent in the standard heterogeneous agent models.

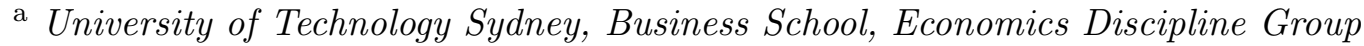 P.O. Box 123, Broadway, NSW 2007, Australia \\ b Division of Economics, School of Humanities and Social Sciences Nanyang Technological University, 14 Nanyang Drive, 637332, Singapore \\ c Amsterdam School of Economics and CeNDEF, University of Amsterdam P.O. Box 15867, 1001 NJ, Amsterdam, The Netherlands}

Keywords: Heterogeneous agent models, discrete choice, switching, experiments. JEL Classification: C25, C91, D83.

\footnotetext{
*We benefited from helpful comments by Simone Alfarano, Cees Diks, John Duffy, Cars Hommes, two anonymous referees, an associate editor, co-editor Nick Vriend, and participants of the 5th POLHIA Workshop 2011 in Paris, France, the Computing in Economics and Finance conference 2011 at San Francisco, USA, the IMEBE meeting 2012 in Castellon, Spain, the 2014 Sydney Economics and Financial Market Workshop, the 2015 WEHIA meeting in Nice, France, and seminars at the Tinbergen Institute and the University of Amsterdam. An earlier version of this paper was circulated under the title "Switching Behavior in the Lab". We are grateful to the financial support from the EU 7th framework collaborative project "POLHIA" Grant No. 225048 and the Research Priority Area Behavioral Economics of the University of Amsterdam. Mikhail Anufriev and Jan Tuinstra acknowledge financial support from the Australian Research Council through Discovery Project DP140103501.

†Corresponding author. E-mail: Mikhail.Anufriev@uts.edu.au.

‡E-mail: Baote@ntu.edu.sg.

${ }^{\S}$ E-mail: J.Tuinstra@uva.nl.
} 


\section{Introduction}

The recent financial crisis has increased interest, both from academics and policy-makers, in agent-based models as a viable alternative to the classic rational choice model. The agent-based model have been relatively successful in describing bubbles and crashes and other stylized facts of financial markets, e.g., excess volatility, volatility clustering and fat tails, see Lux (2009). One important class of agent-based models is that of so-called heterogeneous agent models. ${ }^{1}$ In this paper we contribute to the literature on heterogeneous agent modeling by providing microfoundations for these models through designing and running a novel laboratory experiment with human subjects. This experiment is aimed at validating the assumptions used in the models and calibrating their important parameters. The experimental results reported in this paper can help researchers using heterogeneous agent models and agent-based models by disciplining their choices in modeling and policy exercises.

Heterogeneous agents models assume that there is a large population of traders, with each trader behaving according to one particular trading or forecasting heuristic from a small set of available heuristics (for most applications the number of considered heuristics is only two or three). Past performance of these heuristics then determines the fraction of the population of traders that uses each of the heuristics. This choice process is typically modeled by the so-called discrete choice model. Since both the evolution of asset prices and the performance of the heuristics depend upon the distribution of traders over the heuristics, heterogeneous agent models give rise to low-dimensional, but highly nonlinear, dynamical systems. The interaction between heuristics typically features complex erratic dynamics, in particular when traders are sensitive to performance differentials between heuristics, and may show a striking resemblance with price dynamics observed on actual financial markets. Because heterogeneous agent models only involve a limited number of variables, describing the price and the distribution of traders over heuristics, their properties can still be studied analytically and, in comparison to other, large-scale, agent-based models, the results are typically easier to interpret.

Although heterogeneous agent models have been quite successful in explaining stylized facts of financial markets, they also exhibit an important drawback. The results obtained crucially depend both upon the set of heuristics considered, and on the way the choice between the alternative heuristics is modeled. Indeed, there are many degrees of freedom and ideally heterogeneous agent modeling is disciplined by empirical evidence on which heuristics are used by human decision makers, and how human decision makers switch between those heuristics.

Extensive research on so-called 'Learning-to-Forecast' laboratory experiments already pro-

\footnotetext{
${ }^{1}$ Examples of heterogeneous agent models of financial markets include Brock and Hommes (1998), Brock et al. (2005), Chiarella et al. (2007), Manzan and Westerhoff (2007), Gaunersdorfer et al. (2008), Anufriev and Dindo (2010), Goldbaum and Panchenko (2010) and Anufriev and Tuinstra (2013). For reviews see Hommes (2006, 2013) and LeBaron (2006). Heterogeneous agent (and agent-based) models have also been applied in macroeconomics. The recent review of Fagiolo and Roventini (2016) discusses the issue of calibration and over-parametrization of agent-based models at length. We address this issue using a laboratory experiment.
} 
vides substantial insight into the type of forecasting heuristics that are used by human subjects. $^{2}$ In the current paper we present results from a laboratory experiment that was designed to understand how human decision makers choose between different alternatives, and therefore complements the earlier Learning-to-Forecast experiments. By focusing on the question of how participants use the past performance in switching, we hope to provide further microfoundations for the growing literature on heterogeneous agent models.

In particular, we are interested in finding a specification of the discrete choice model that is able to explain participants' decisions satisfactorily. In addition we will try to infer the appropriate value of the so-called Intensity of Choice (IoC) parameter of the discrete choice model. This parameter measures how responsive traders are with respect to differences in past performance of the heuristics and it plays a pivotal role in the dynamic properties of heterogeneous agent models. In particular, low values of the IoC are associated with stable dynamics, whereas high values typically lead to complicated phenomena and endogenous fluctuations in stock prices, volumes and trading positions. Learning the relevant range of values of the IoC is therefore crucial for a proper application of heterogeneous agent models. However, significant estimates of this parameter are difficult to obtain from empirical studies because, due to a lack of direct access to the strategies used, switching behavior can only be inferred indirectly.

Our laboratory experiment is designed such that it facilitates estimating the parameters of the discrete choice models. The advantage of running a laboratory experiment is that it provides a controlled environment where we can observe the choices of the participants directly. We study the switching behavior of human subjects between a small number of alternatives. The participants observe the past performance (framed as "financial returns") of several (either two, three or four) investment alternatives ("funds") and, in every period, are asked to choose one of the alternatives and are paid on the basis of the performance of the chosen alternative in that period. As in actual financial markets, participants do not know the data generating process of the returns, and their choice does not influence the return of the different alternatives. ${ }^{3}$ We use different data generating processes that result in differences in the autocorrelation structure (and therefore predictability) of the generated time series. Participants have to choose between funds for 40 consecutive periods for one set of funds, and then for another 40 periods for a different set of the funds. This allows us to study the effect of experience on choice behavior.

We find that participants often switch between funds. Given the information provided in the experiment, it is not surprising that switching is, to a large extent, driven by past performance of the funds. Answering the question of how exactly switching is driven by past performances, we find that a simple discrete choice model with a predisposition effect provides a good fit to the data, when there are two or three funds and there is no cyclical/periodic pattern in the time series of returns. If such a cyclical pattern does exist, more lags need to be incorporated in the model in order to provide a good description of the data. This suggests

\footnotetext{
${ }^{2}$ See Hommes et al. (2005), Heemeijer et al. (2009), Bao et al. (2012), Pfajfar and Žakelj (2014) and Bao et al. (2016). The review in Hommes (2011) provides additional references.

${ }^{3}$ Anufriev et al. (2015) discuss a related experiment where subjects do know the data generating process.
} 
that participants recognize and exploit cyclical patterns in returns. Moreover, the estimated IoC is not universal, but positively related to the degree of autocorrelation in the time series of returns. From this we conclude that participants do not myopically chase past returns, but are more likely to do so when past returns are more predictive of future returns. Our paper therefore also contributes to the empirical literature that suggests that the flows in and out of mutual funds are to a substantial extent driven by the recent past performance of these funds, despite the fact that the funds which performed well in the past do not necessarily generate an above-average return. ${ }^{4}$ By focusing participants' attention on the funds' past performance, our experiment directly addresses the question of how past performance affects their choices. ${ }^{5}$

The remainder of the paper is organized as follows. Section 2 presents the discrete choice framework and discusses some related literature. Section 3 introduces the experimental design and Section 4 provides a first discussion of the experimental results. In Section 5 we estimate the binary choice model on the experimental data from sessions with two funds. Section 6 discusses the estimated discrete choice models for sessions with more than two funds. Section 7 discusses the results. Appendices A, B and C contain the experimental instructions, further details of the experimental design, and some estimation results. Appendices D, E and F contain a detailed explanation of our estimation procedures and some further statistics.

\section{Switching and the Discrete Choice Framework}

Although not the first to explain market fluctuations by the interaction of different heuristics, arguably the two seminal papers in the field of heterogeneous agent models are Brock and Hommes (1997) and Brock and Hommes (1998). ${ }^{6}$ Brock and Hommes (1997) consider the classic cobweb model where suppliers can either form price expectations rationally (against positive information costs) or employ (cost free) naive expectations, i.e., predicting that the price will equal the last observed realized price. Total supply, and therefore the market clearing price, will then depend upon a weighted average of the rational and naive forecasts. The evolution of the fraction of the population of firms using rational expectations is modeled by the discrete choice dynamics with forecasting accuracy as the performance measure. The paper shows that if the Intensity of Choice (IoC) is large enough, the dynamics converges to a strange attractor with endogenous fluctuations in the market price. Moreover, the naive expectations heuristic survives the competition with the more sophisticated rational expectations heuristic.

\footnotetext{
${ }^{4}$ See Sirri and Tufano (1998), Zheng (1999), Jain and Wu (2000), Choi et al. (2009) and Ferreira et al. (2012) among others. In addition, Choi et al. (2009) find that "return chasing behavior" also describes investment behavior in the 401(k) accounts by American households quite well.

${ }^{5}$ In fact, it is very common for professional financial websites (e.g., Morningstar, Yahoo Finance) to provide information on past performance of all mutual funds, and recommend or rate funds based upon this past performance.

${ }^{6}$ According to Web of Science (Google Scholar) together the two papers have been cited more than 1000 (2600) times by April 12, 2016. Important earlier influential contributions include Day and Huang (1990), Chiarella (1992) and Lux (1995).
} 
The same notion of (evolutionary) competition between different heuristics was applied to financial markets in the follow-up paper, Brock and Hommes (1998), where traders can choose between different forecasting strategies representing, for example, fundamentalist traders, chartists and noise traders. Again, if the IoC parameter is large enough, the asset price dynamics exhibit irregular switches between periods of prolonged deviations from the fundamental value followed by a correction in the direction of that fundamental value. This model has given rise to a huge stream of theoretical, computational and empirical studies on endogenous dynamics in financial markets. The key mechanism in these models, responsible for the non-linearities leading to excess volatility, endogenous bubbles and crashes, etc., is the discrete choice model which describes the evolution of the fractions of traders using the different heuristics by the multinomial logit model. ${ }^{7}$ In its most basic version the discrete choice model assumes that the fraction $n_{t}^{h}$ of the population of decision makers using heuristic $h$ in period $t$ is given by

$$
n_{t}^{h}=\frac{\exp \left[\beta \pi_{h, t-1}\right]}{\sum_{k=1}^{H} \exp \left[\beta \pi_{k, t-1}\right]} .
$$

Here $\pi_{h, t-1}$ is the payoff or profit that was generated by heuristic $h$ in period $t-1$ and $\beta>0$ is the Intensity of Choice parameter that was already discussed above. If this parameter is small (e.g., close to zero) the population of decision makers is spread out almost evenly over the different heuristics, whereas a very high value of $\beta$ implies that decision makers are sensitive to differences in payoffs and the heuristic with the highest payoff attracts the majority of the population of decision makers.

In this paper we will argue on the basis of experimental evidence that the following, more general, version of (1), can be a more realistic choice in modeling the evolution of fractions:

$$
n_{t}^{h}=\frac{\exp \left[\alpha_{h}+\beta_{h, 1} \pi_{h, t-1}+\ldots+\beta_{h, L} \pi_{h, t-L}\right]}{\sum_{k=1}^{H} \exp \left[\alpha_{k}+\beta_{k, 1} \pi_{k, t-1}+\ldots+\beta_{k, L} \pi_{k, t-L}\right]} .
$$

This specification generalizes (1) in three directions. First, it allows for up to $L$ lags of the payoffs to play a role in the determination of the fraction choosing heuristic $h$. Second, it does not require that decision makers respond to payoffs of the different heuristics in the same manner. That is, we can have $\beta_{k, \ell} \neq \beta_{h, \ell}$ for some lag $\ell$ and some heuristics $h$ and $k$ with $k \neq h$. Third, it allows for a so-called predisposition effect: if $\alpha_{h}>\alpha_{k}$ for some $h$ and all $k \neq h$, then traders are, on aggregate, biased towards choosing heuristic $h$ over the other heuristics, even when there is no difference in the past performance of these heuristics. ${ }^{8}$

A vast majority of the heterogeneous agent models uses equation (1) to model the fraction of the population of traders that selects heuristic $h$ in period $t$. As discussed above, the dynamic properties of these models depend critically upon the choice of the IoC parameter

\footnotetext{
${ }^{7}$ The discrete choice model has its origin in econometrics, see Manski and McFadden (1981) for an overview and a discussion of the underlying assumptions.

${ }^{8}$ Other generalizations are possible as well, see, e.g., Anufriev and Hommes (2012b) who consider a discrete choice model with memory in the performance measure (corresponding to geometrically declining weights on lagged payoffs) and asynchronous updating.
} 
$\beta$ and therefore several researchers have attempted to estimate $\beta$, typically on the basis of market or survey data. Branch (2004), for example, uses survey data on inflation expectations from Michigan households to estimate the discrete choice model with three forecasting heuristics. His estimated model gives a good description of the data, with the qualification that individuals typically exhibit a predisposition effect as captured by differences in the estimated values of the $\alpha$ parameters in equation (2). Boswijk et al. (2007) estimate model (1) and the parameters of two heuristics on yearly S\&P500 data from 1871-2003. Although the estimated IoC is not statistically significant, their model can explain the price bubble from the late nineties. Empirical evidence of switching (i.e., an estimated IoC significantly higher than zero) between three heuristics on the interlinked stock markets of Hong Kong and Thailand is presented in De Jong et al. (2009). An alternative heterogeneous agent model is estimated on monthly S\&P500 data from 1970-2012 by Chiarella et al. (2014). They also find evidence for switching between fundamentalism and trend-following behavior. Goldbaum and Mizrach (2008) make a distinction between passively and actively managed mutual funds and estimate the IoC parameter from data on the flows of money into and out of these mutual funds. Franke and Westerhoff (2012) propose a method of simulated moments approach to compare how well different types of agent-based models perform in describing stylized facts of financial markets ${ }^{9}$ and find that inclusion of switching based upon the discrete choice mechanism (1) improves data fitting. Finally, Anufriev and Hommes (2012a,b) calibrated a so-called 'Heuristic Switching Model', which is similar to the multinomial logit model given by (1), on the experimental data obtained from 'Learning-to-Forecast' experiments.

One drawback of all of this empirical and experimental work is that neither the specific heuristics nor actual switches from one heuristic to another are observed, and therefore have to be inferred from the data. In our experiment, on the other hand, the heuristics are given (and represented by different investment alternatives) and we observe precisely when participants switch from one alternative to another. In fact, the common denominator of all of the literature referenced above is that none of these studies have attempted to estimate the IoC parameter from the choices participants make directly. The aim of the current paper is to fill that void. ${ }^{10}$

\footnotetext{
${ }^{9}$ Some agent-based models explain the excess volatility and other stylized facts without modeling switching between alternative heuristics on the basis of past performance. To generate volatility these models either impose Markov regime switching of fundamental value as in Chiarella et al. (2012), or model herding through imitation learning as in Alfarano et al. (2008).

${ }^{10}$ Our experiment is somewhat related to experimental studies on so-called "information cascades", which can also explain herding behavior on financial markets, see Anderson and Holt (1997), Drehmann et al. (2005) and Alevy et al. (2007). In these experiments participants choose between two assets and receive a private as well as a public signal about which asset has the highest payoff, where the public signal consists of the earlier choices of the other participants. An information cascade sets in when participants base their decision purely on the public signal and disregard their private signal, even if the signals are conflicting. Anderson and Holt (1997) estimate a discrete choice model on the probability of choosing a particular asset and find an IoC that typically lies between 2 and 8 . While in their experiment different participants play in different periods and the distribution of payoff of the assets is fixed, in our experiment the same participant makes decisions for all periods, but the payoffs of the assets are time varying.
} 


\section{Experimental Design}

The experiment was conducted on June 17-18, 2010, at the CREED laboratory of the University of Amsterdam. A total of 91 students participated in four sessions comprising the six different treatments explained in Section 3.3 below. None of the students participated in more than one session. Each session lasted for about one hour and a half, and the participants' payoffs varied between 18 and 25 euros.

\subsection{Participants' task}

The experiment is an individual choice experiment where participants have to make an investment decision repeatedly. The participants observe the time series of past returns of either two, three or four investment alternatives, represented as 'funds' A, B, C and D, in two blocks of 50 periods. The type and number of investment alternatives are different in the two blocks. Each block starts with a history of 10 periods shown to the participant, so that (s)he can get acquainted with the investment alternatives. For each of the remaining 40 periods in that block the task of the participant is to choose one of the investment alternatives. After the choice is made the realized returns for that period of all of the available investment alternatives are revealed.

The payoff for a participant in any particular period is equal to the return of the alternative (s)he chooses in that period. This return per period ranged between 0 and 16 points. The total earnings of each participant was determined as the total number of points earned during the experiment $^{11}$ (i.e., over $2 \times 40=80$ decision periods), translated to euros by dividing the total number of points by 20. In addition, participants receive a 'show-up' fee of 5 euro.

Fig. 1 shows an example of the computer screen the participants face during the experiment. ${ }^{12}$ A participant sees the past returns of all the funds on a graph as well as in a table, where also the own past choices are displayed. The participant has to choose which fund to invest in, using the radio buttons in the top part of the screen. After the participant has chosen the fund, the computer screen is updated with the new information.

The instructions are provided in Appendix A. Note that the participants did not know the data generating process that underlies the time series they observe, but they knew that a different data generating process is used in the second block of 50 periods.

\footnotetext{
${ }^{11}$ We have chosen this payment scheme over the alternative where a participant is paid for a few random periods to avoid that risk-averse participants choose the find with the lowest variance, instead of the fund with the highest expected return. Our payoff scheme is consistent with the typical random utility setup used in the literature on heterogeneous agent models, where agents are motivated to choose an alternative with maximum expected payoff in the current period.

${ }^{12}$ The time series shown in the screen of the figure have not been used in the experiment. This picture was printed on the paper shown to the participants during the instruction stage of the experiment, to explain the different parts of the screen.
} 


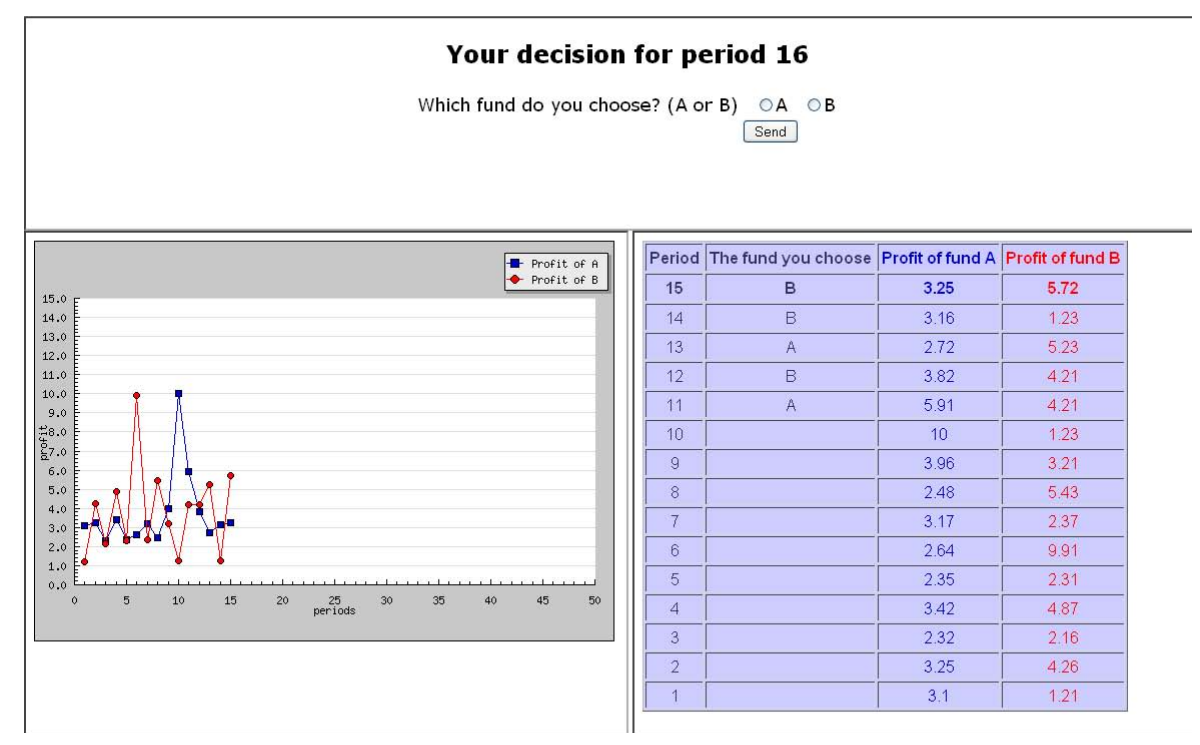

Figure 1: An example of the experimental screen. Participants submit their decisions by selecting the radio buttons in the upper part of the screen.

\subsection{Time series used in the experiment}

Prior to the experiment we prepared seven sets of time series of 50 periods, representing returns (or payoffs) of the different investment alternatives. Letting $t$ denote the period, each time series is defined for $t=-9, \ldots, 40$. The first 10 realizations, corresponding to $t \leq 0$, are shown to the participants at the start of the relevant block. The remaining observations are shown sequentially as decisions are being made, such that for each time period $t, t=1, \ldots, 40$, in which participants have to make an individual investment decision, they can observe return realizations from periods $\tau=-9, \ldots, t-1$, for all investment alternatives in the relevant set.

The time series were generated using the data generating processes (DGPs) discussed below and then grouped in sets of two, three or four time series. The sets used in different blocks of the experiment are illustrated in the left panels of Figs. 2, 3 and 4. At the start of every block the participants could see the part of the time series to the left of the vertical dotted line. ${ }^{13}$ The sets of time series can be characterized by their autocorrelation properties. Since we conjecture that for a participant's decisions the differences between returns of different alternatives are more important than the absolute value of these returns, we specifically look at the autocorrelation structure of return differences. Taking alternative A as a benchmark, we calculate the return difference as the return of A minus the return of one of the other alternatives. The autocorrelation function is plotted until the 10th lag. The dashed lines represent 95\% confidence bands.

\footnotetext{
${ }^{13}$ The line was not shown in the experiment.
} 

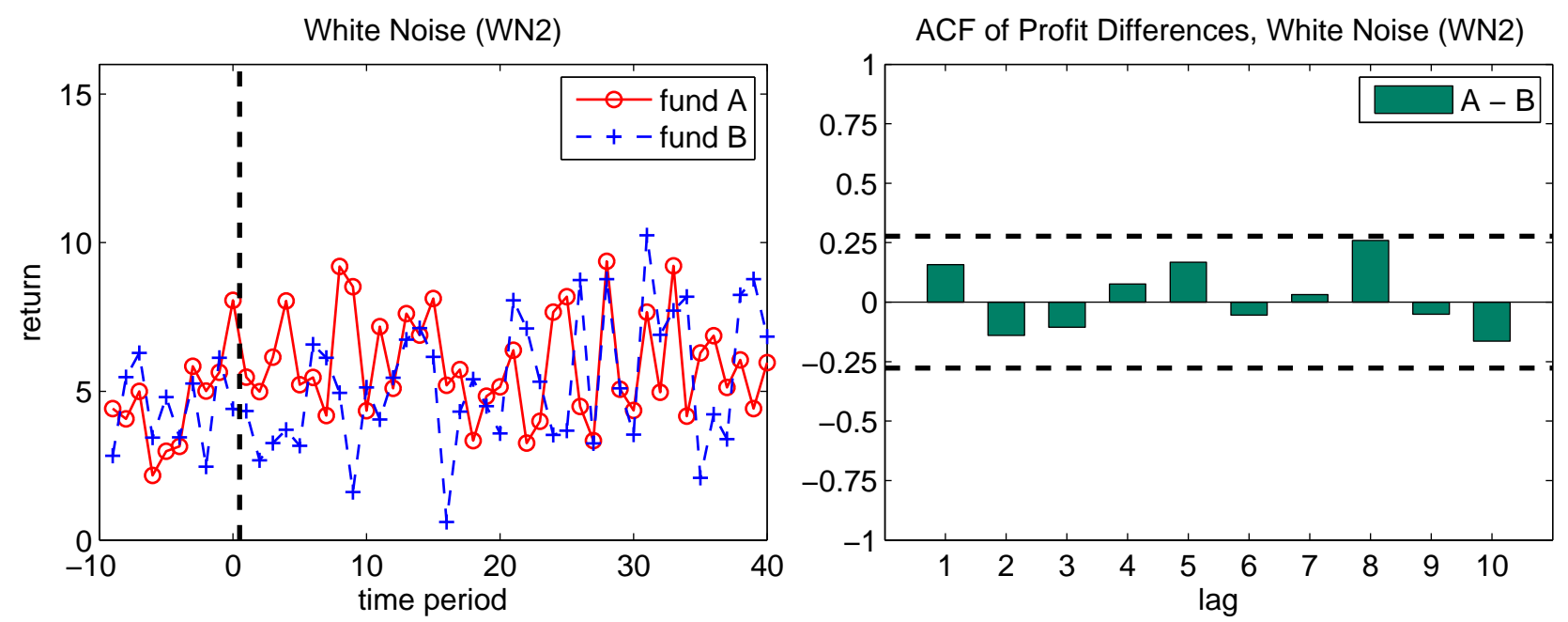

Figure 2: The return time series generated by the white noise model, WN2 (left panel) and the autocorrelation of the difference between the returns (right panel).

1. White Noise time series. The first set of time series, denoted WN2, are generated by the following DGP:

$$
\pi_{i, t}=5+\varepsilon_{i, t}, \quad i=A, B \quad \text { and } \quad t=-9, \ldots, 40,
$$

where the $\varepsilon_{i, t}$ are independently and identically distributed, with $\varepsilon_{i, t} \sim N(0,1)$. The time series used in the experiment are shown in Fig. 2 together with the autocorrelations of the difference $\pi_{A, t}-\pi_{B, t}$. These two time series are independent from each other and serially uncorrelated.

2. Brock-Hommes time series. Three sets of time series, labeled BH2, BH3 and BH4, are generated from numerical simulations of the two canonical heterogeneous agent models of Brock and Hommes $(1997,1998)$ described in the previous section. The resulting sets of time series are shown in Fig. 3 (left panels). Appendix B provides the details of the data generating processes.

As explained in the previous section, Brock and Hommes (1997) introduce switching between heterogeneous expectations in the cobweb model. In particular, they study the dynamics of the model with two types of expectations, costly rational expectations and costless naive expectations. Our set BH2 contains the two time series of the realized returns associated with these two forecasting heuristics, for a particular parameter setting. ${ }^{14}$ The participants to the experiment experience this as a decision problem where there are two funds available for investment: fund A and fund B. Due to the nonlinear nature of the model, the simulated time series exhibit a quasi-cyclic pattern, with fund A performing slightly worse than fund B most

\footnotetext{
${ }^{14}$ Note that in the Brock-Hommes model the distribution of the population over the different heuristics is one of the determinants of prices and profits. In the experiment, however, choices of the participants have no effect on the actual payoffs. We leave the experimental study of choices between heuristics, where these choices feed back into the determination of profits, for future research.
} 

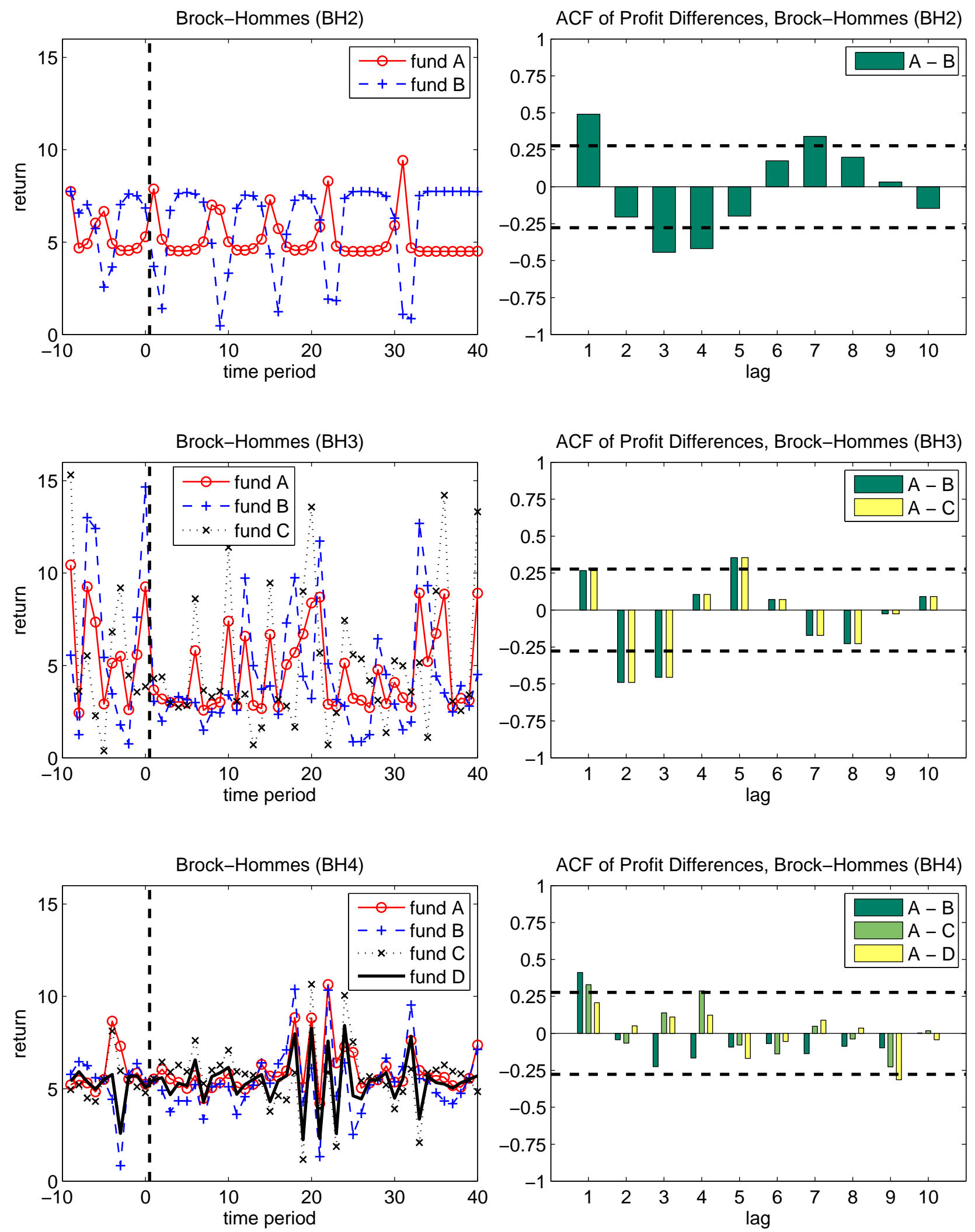

Figure 3: The return time series generated by the Brock-Hommes models (left panels) and the autocorrelations of the differences between the returns (right panels). 
of the time but strongly outperforming fund B at certain periods. The autocorrelation function shown in the right upper panel of Fig. 3 reflects this pattern with significant autocorrelations at lags 1, 3, 4 and 7. Despite the quasi-periodic pattern, the time series are chaotic. In other words, for the participants it is hard to predict the precise moment when fund A performs better than fund B.

The next two sets of time series, BH3 and BH4, are based on Brock and Hommes (1998), who apply the idea of evolutionary switching between heterogeneous forecasting heuristics to a financial market model and study the dynamics for several versions of their model. The three time series in BH3 are generated by the model with three forecasting heuristics, where one of the heuristics corresponds to fundamentalist behavior, and the two others represent opposite biased predictors. The returns of fund A are those generated by the fundamental forecast, whose performance is always in between the performances of the two other funds. Funds B and $\mathrm{C}$ are symmetric with respect to $\mathrm{A}$, as their (opposite) biases are the same in absolute value. From time to time a different fund performs best. Fig. 3 (middle right panel) shows the autocorrelations of $\pi_{A, t}-\pi_{B, t}$ and $\pi_{A, t}-\pi_{C, t}$, which are identical due to symmetry in the model. There is no apparent structure in the returns. ${ }^{15}$

The four time series in BH4 are also generated by the asset pricing model from Brock and Hommes (1998) but with four heuristics, representing fundamentalists, trend chasers with an upward bias, trend chasers with a downward bias and strong trend chasers with no bias, respectively Also for this set of time series the autocorrelation structure in differences is not too strong, although it is significant for the first lag (for $\pi_{A . t}-\pi_{B, t}$ and $\pi_{A, t}-\pi_{C, t}$ ).

3. Stock Index time series. These sets, labeled SI2, SI3 and SI4, are constructed from four actual stock indices, the Austrian Trade Index (ATX), the Belgium 20 Stock Index (BFX), the Dow Jones Index (DJI) and the FTSE 100 index (FTSE), from October 2005 to November 2009. We use rotation based yearly returns, instead of monthly returns. ${ }^{16}$ We use the ATX and BFX indices for SI2, the ATX, BFX and DJI indices for SI3, and the ATX, BFX, DJI and FTSE for SI4. Further, we normalize the data by dividing the returns by 12 and adding 5 . The resulting time series are shown in the left panel of Fig. 4. All of them are highly correlated as can be seen from the right panels of Fig. 4. Autocorrelations of the difference between funds $\mathrm{A}$ and $\mathrm{B}$, between funds $\mathrm{A}$ and $\mathrm{C}$ and between funds $\mathrm{A}$ and $\mathrm{D}$ are significant up to lag $4, \operatorname{lag} 6$ and lag 7 , respectively.

By applying a suitable linear transformations to the BH and SI sets, we made sure that the maximum, minimum and average returns are similar across time series. This is necessary

\footnotetext{
${ }^{15}$ At the $95 \%$ confidence level the autocorrelation in differences is significant only at lags 2,3 and 5 (but not at the first lag).

${ }^{16}$ Due to using rotation based returns, these time series do not look like the typical monthly returns of mutual funds. We label them here as "stock index" only due to the source of the data. The main purpose of using these time series is to study switching behavior when returns are highly autocorrelated. The results from the white noise time series may have more relevance for the behavior of investors in actual markets for mutual funds.
} 
Stock Indices (SI2)

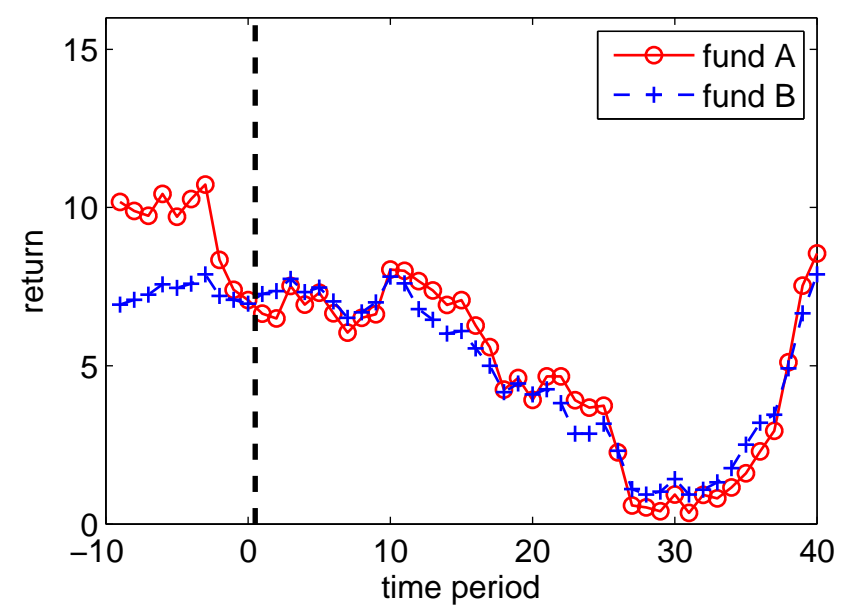

Stock Indices (SI3)

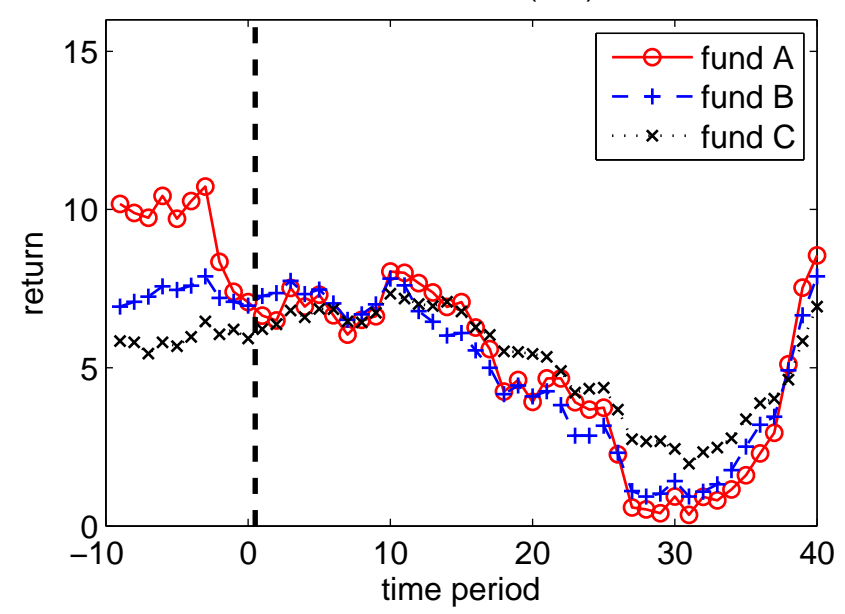

Stock Indices (SI4)

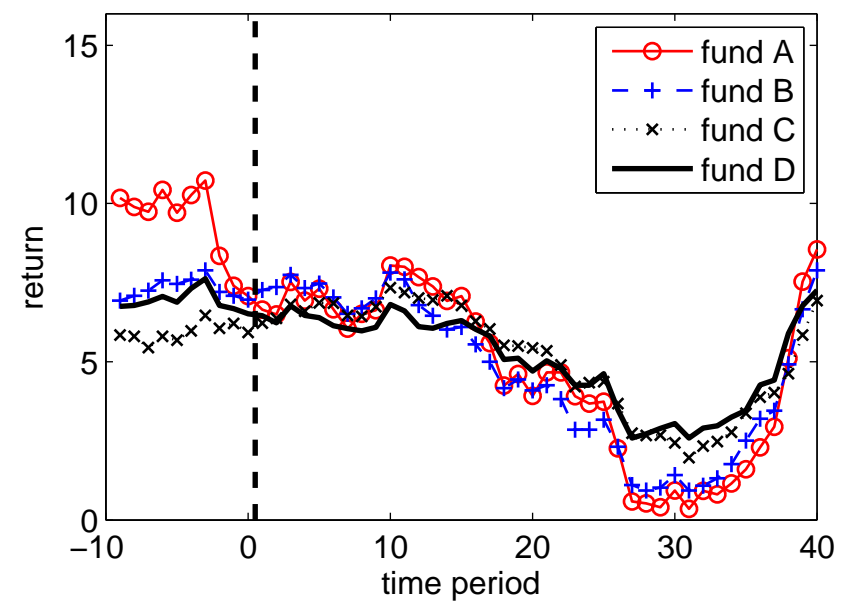

ACF of Profit Differences, Stock Indices (SI2)

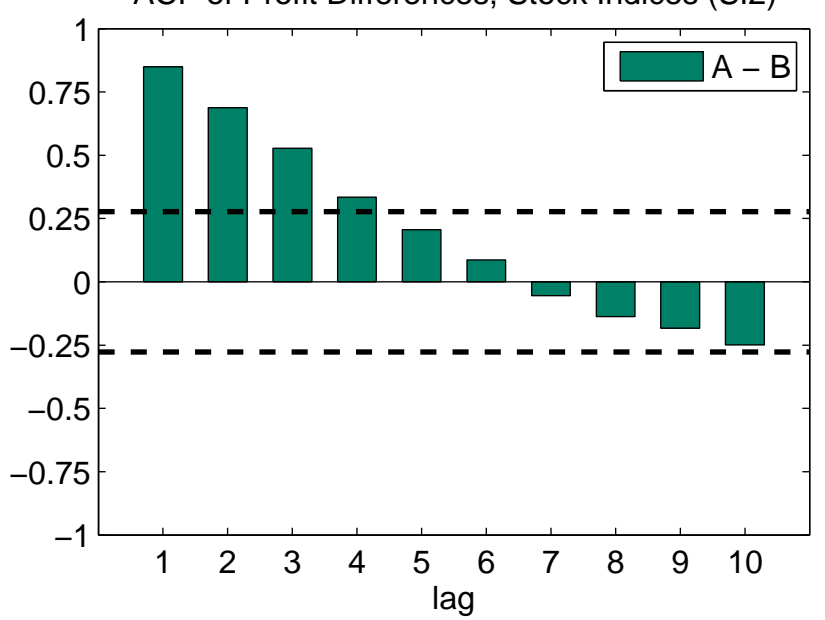

ACF of Profit Differences, Stock Indices (SI3)

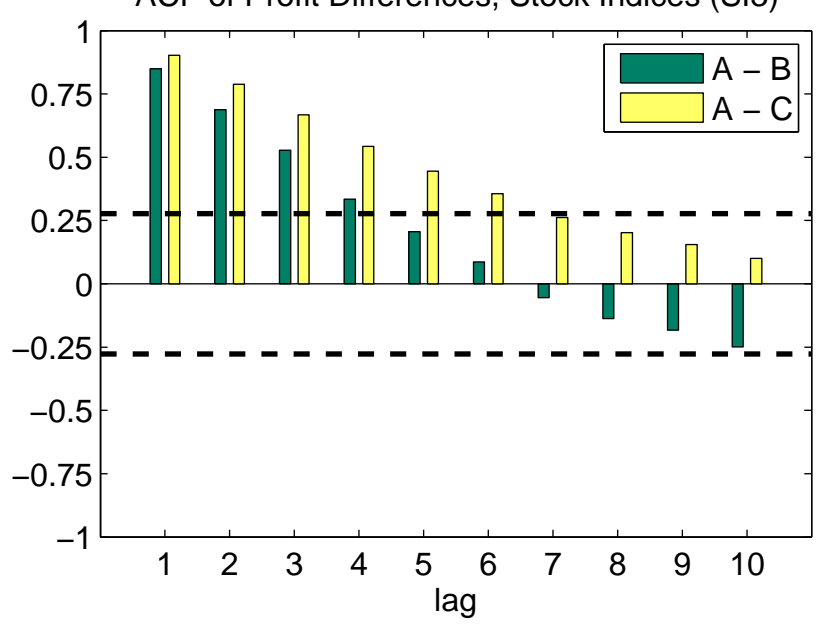

ACF of Profit Differences, Stock Indices (SI4)

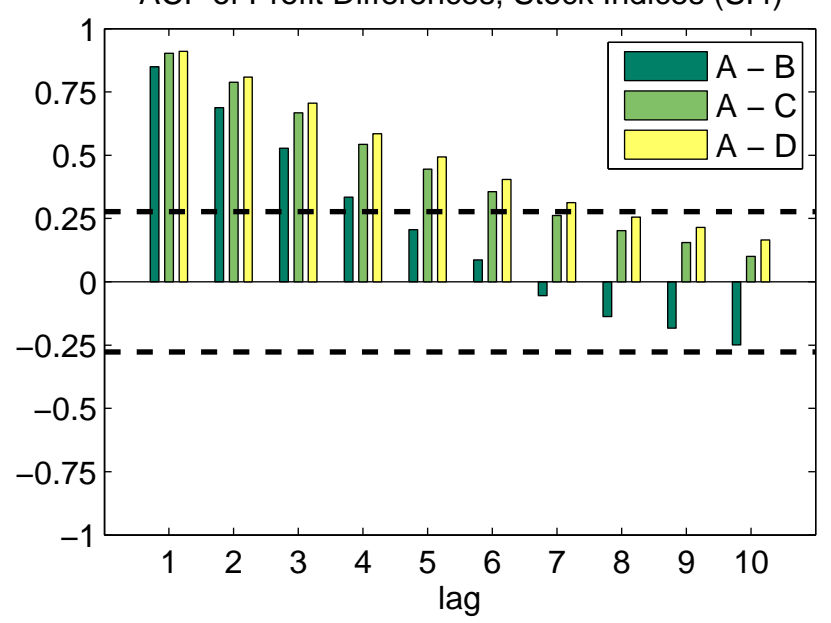

Figure 4: The return time series of the stock indices (left panels) and the autocorrelations of the differences between the returns (right panels). 


\begin{tabular}{c|c||c|c|c}
\hline \multirow{2}{*}{ Time Series } & \multirow{2}{*}{ Treatments } & \multicolumn{3}{c}{ Number of participants } \\
\cline { 3 - 5 } & & Block 1 & Block 2 & Total \\
\hline WN2 & T1 and T6 & 16 & 13 & 29 \\
BH2 & T2 and T5 & 15 & 14 & 29 \\
BH3 & T3 and T1 & 14 & 16 & 30 \\
BH4 & T4 & - & 14 & 14 \\
SI2 & T4 and T3 & 14 & 14 & 28 \\
SI3 & T5 & 14 & - & 14 \\
SI4 & T6 and T2 & 13 & 15 & 28 \\
\hline
\end{tabular}

Table 1: Structure of the treatments and blocks.

in order to compare the estimated intensity of choice parameters across different sets of time series. ${ }^{17}$ The descriptive statistics of all time series is provided in Table 5 in Appendix B.

Time series from the different sets differ substantially in their autocorrelation structure. The time series from set WN2 exhibit almost no autocorrelation or periodic pattern. The time series from sets $\mathrm{BH} 2$ and BH3 exhibit a strong quasi-periodic pattern. Set BH4 has a high autocorrelation only for the first lag. Finally, the time series in sets SI2, SI3 and SI4 exhibit very strong autocorrelations.

\subsection{Treatments}

After generating the seven sets of time series, we constructed six treatments. Each treatment consists of two blocks, and in each block a participant is confronted with one of the possible seven sets of time series. Of the $7 \times 6=42$ possible combinations of two different sets, we selected six, under the restrictions that the two blocks in a treatment had to consist of sets with both a different number $(2,3$ or 4$)$ and a different type (WN, BH or SI) of investment alternatives. Moreover, in order to test the effect of experience with another set of time series, we organized the treatments in such a way that all the sets (except BH4 and SI3) appear exactly once in the first and once in the second block. The participants who have a given set of time series in their first block are denoted "inexperienced" (with this set) and those who have the set in their second block as "experienced".

The six resulting treatments $\mathrm{T} 1$ - T6 have the following sequences of sets of time series: WN2 $\rightsquigarrow \mathrm{BH} 3$ (T1), BH2 $\rightsquigarrow \mathrm{SI} 4$ (T2), BH3 $\rightsquigarrow \mathrm{SI} 2$ (T3), BH4 $\rightsquigarrow \mathrm{SI} 2$ (T4), SI3 $\rightsquigarrow \mathrm{BH} 2$ (T5) and SI4 $\rightsquigarrow$ WN2 (T6). Table 1 is organized by the sets of the time series and shows the design and the number of participants for each of the time series.

\footnotetext{
${ }^{17}$ The intensity of choice in (1) is not a scale-free parameter: multiplying all payoffs, $\pi_{k, t-1}$ for all $k$, by a constant is equivalent with dividing the estimated intensity of choice parameter by that same constant.
} 


\subsection{Hypotheses}

In the remainder of the paper we will present and analyze the experimental results. We present the data in Section 4.1 and then focus on five different hypotheses which are introduced below.

First, all time series except BH4 and SI2 have been presented to participants of two treatments, once in the first block and once in the second block (see Table 1). The question then arises if, for a given set of time series, inexperienced and experienced participants behave similarly. In Section 4.2 we test the following hypothesis.

Hypothesis 1. Behavior of participants for a given set of time series is independent of whether they experience this set in the first or in the second block of the experiment.

If Hypothesis 1 is confirmed, we can pool, for a given set time series, the data from participants that received this set in the first block with the data from participants that received the set in the second block.

Turning to the main goal of the analysis, in Section 4.3 we present qualitative evidence that participants condition their decisions on payoff information from the previous period, i.e., that they exhibit return chasing. An important question of how they chase returns is rigorously studied by estimating discrete choice models in Sections 5 and 6. Discrete choice models, as they are used in the literature with heterogeneous agents, are backward looking models, i.e., it is assumed that past returns determine the fractions of the population choosing the different alternatives. In terms of the estimated discrete choice model, return chasing is represented by the following hypothesis.

Hypothesis 2. Estimating model (1) or (2) on aggregate data gives estimates of $\beta$ or $\beta_{h, 1}$ (for all $h$ ) that are positive and significantly different from 0 for all sets of time series.

Confirmation of Hypothesis 2 would suggest that participants indeed exhibit return chasing behavior.

The discrete choice model (1) furthermore assumes that agents only respond to the level of the differences in past returns and not to patterns in these differences. The implication of this is that there should be no significant differences between the discrete choice models that are estimated from the experimental data generated by different time series.

Hypothesis 3. Experimental data from different sets of time series can be explained by exactly the same discrete choice model.

If Hypothesis 3 is rejected, a universal discrete choice model - and therefore a universal value of the intensity of choice $\beta$ - does not exist. The responsiveness of participants with respect to past payoff differences may then vary with the economic environment or with, for example, observable patterns in past returns. 
Moreover, discrete choice model (1) assumes that returns from the previous period determine the current fractions, but returns from the more distant past do not play a role. Although longer memory can easily be incorporated in the discrete choice model, most heterogeneous agent models rule this out, typically for reasons of tractability. The following hypothesis can be used to test whether this restriction is appropriate.

Hypothesis 4. A discrete choice model with more lags does not provide a substantially better description of the aggregate data than model (1) (or (2) with $L=1$ ).

If Hypothesis 4 is confirmed, a simple model with one lag will be a reasonable compromise between tractability and realism.

Hypotheses 2, 3 and 4 are tested in Section 5 on the data from the blocks with two alternatives. Most applications of discrete choice models to heterogeneous agent models involve the choice between either two or three alternatives. In Section 6 we will consider the blocks with time series of three or four alternatives. It turns out that the general discrete choice model becomes more complicated when there are more than two alternatives. It is important, therefore, to understand if a fundamentally different model is needed to describe participants' behavior if the number of alternatives increases. We formulate this question as the following hypothesis.

Hypothesis 5. There is no substantial difference in the structure of the best fitted discrete choice model, when the number of alternatives goes from two to three to four.

Rejection of this hypothesis would mean that more sophisticated discrete choice mechanism should be used in the models with more than two alternatives.

\section{Experimental Data: Some Preliminary Findings}

In this section we will have a first look at the experimental data. These data are presented in Section 4.1. In Section 4.2 we investigate the effect of experience and in Section 4.3 we investigate qualitatively whether choices of participants in the experiment are driven by past returns, before we consider this issue in more detail when estimating discrete choice models in Sections 5 and 6 .

\subsection{Experimental data}

Fig. 5 gives, for each set of time series, the evolution of the share of participants choosing a particular alternative. To facilitate exposition, and in anticipation of our first result below, we pooled together participants from two blocks (that is, experienced and inexperienced) for the time series from WN2, BH2, BH3, SI2 and SI4. Clearly, there are substantial fluctuations in 

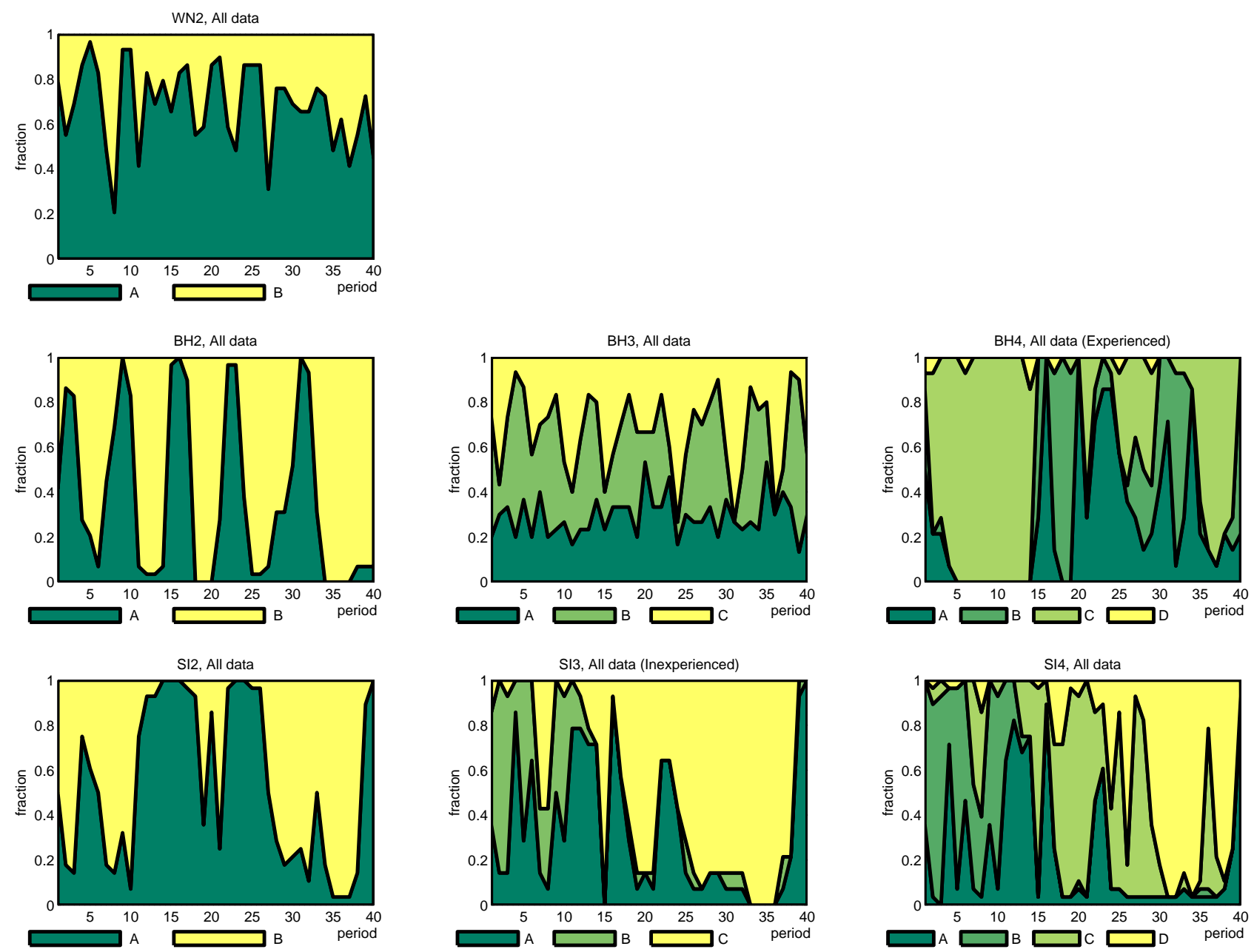

Figure 5: Shares of choices over time for different sets. For WN2, BH2, BH3, SI2, and SI4 the data from the two blocks are pooled.

the shares for a given set of time series, and many participants switch between alternatives regularly during each block.

In addition, there also exist pronounced differences in choice behavior between different sets of time series. In the BH2 blocks, for example, there are large swings in the fraction of participants choosing alternative A (such large swings are also observed in the SI2 blocks, although the pattern is different). In the BH3 blocks, however, the fraction of participants choosing a particular alternative is more stable over time and subject to smaller fluctuations (the same holds for the WN2 blocks).

Closer inspection of the dynamics of fractions reveals some other remarkable characteristics. For example, although expected returns for alternatives $\mathrm{A}$ and $\mathrm{B}$ are the same in the WN2 blocks, participants choose $\mathrm{A}$ with a higher frequency than $\mathrm{B}$ in those blocks. This may be attributed either to a "default choice bias" for alternative $\mathrm{A}^{18}$ or to the realization of the random white-noise process where alternative A has a higher return than alternative B for 7 out of the

\footnotetext{
${ }^{18}$ However, note that in treatment $\mathrm{T} 1$ the participants experienced the BH3 time series after the WN2 time series. In the BH3 block the participants actually choose B more often.
} 
first 10 experimental periods (i.e., for $t=1, \ldots, 10$ ), or a combination of the two. Also, for the set of time series in SI4, the majority of participants learns to switch from alternatives A and $\mathrm{B}$ (which are the most profitable alternatives in periods $t=-9, \ldots, 5$ ) to alternatives $\mathrm{C}$ and $\mathrm{D}$ (which are, on average, the most profitable alternatives in periods $t=17, \ldots, 37$ ). These observations suggest that participants do positively respond to past profit differences. We will return to this issue in Section 4.3, but first we investigate the effect of experience.

\subsection{Effect of experience}

We analyze the effect of experience with another set of time series by comparing the aggregate behavior of participants who experience a particular set of time series in the first block of their treatment to those who experience that set of time series in the second block of their treatment. Given the design of treatments (see Table 1) we can do this for the WN2, BH2, BH3, SI2 and SI4 blocks, but not for the BH3 and SI4 blocks. ${ }^{19}$ Since by the design of the experiment the choices of individual participants are independent from each other for any given time period, the distribution of choices is multinomial. We can therefore apply Pearson's chi-squared test for each of the five relevant sets of time series and for every $t=1, \ldots, 40$ to test the hypothesis that choice behavior of experienced and inexperienced participants is the same. We only reject this hypothesis for five of the $5 \times 40=200$ periods, see Appendix D for details.

An alternative way to investigate the effect of experience is by determining, for each participant, how often he/she switches between alternatives during a block. Subsequently, we compare the empirical distribution of the number of switches of inexperienced participants to that of experienced participants. Using the Kolmogorov-Smirnov test we can reject the hypothesis that these distributions are the same only for the SI2 blocks. ${ }^{20}$

Summarizing, we reach the following conclusion.

Result 1. We confirm Hypothesis 1. For a given set of time series choice behavior of experienced participants is not significantly different from that of inexperienced participants.

This result is corroborated by a comparison of the performance of inexperienced participants with that of experienced participants. Table 2 shows, for each set of time series, how often participants make the optimal decision by choosing the alternative with the highest actual return in that period. Except for the WN2 time series, the fractions are much higher than what they would be under random choice (i.e., $50 \%$, $33 \%$ or $25 \%$ for time series with two, three and four funds, respectively), see the third column of Table 2. Participants are most successful

\footnotetext{
${ }^{19}$ Fig. 9 in Appendix D shows the figures for each block separately, which facilitates a comparison of the behavior of experienced vs. inexperienced participants.

${ }^{20}$ The test statistics are 0.375 for WN2, 0.176 for BH2, 0.5 for SI2, 0.188 for BH3 and 0.185 for SI4. The corresponding $p$ values are $0.207,0.962,0.039,0.930$ and 0.952 , respectively. Fig. 9 in Appendix D shows the histograms of the number of switches for inexperienced and experienced participants. Table 12 in Appendix D shows, for each block, the number of switches per participant per period.
} 


\begin{tabular}{c|c||c|cc|cc}
\hline \multirow{2}{*}{ Time series } & \multirow{2}{*}{ Treatments } & \multicolumn{5}{c}{ Fraction of Best Choices } \\
\cline { 3 - 7 } & & Total & Inexp. & Exp. & First 20 & Last 20 \\
\hline WN2 & T1 and T6 & $52.24 \%$ & $53.13 \%$ & $51.15 \%$ & $57.41 \%$ & $47.07 \%$ \\
BH2 & T2 and T5 & $83.10 \%$ & $81.33 \%$ & $85.00 \%$ & $79.14 \%$ & $87.07 \%$ \\
BH3 & T3 and T1 & $44.58 \%$ & $46.25 \%$ & $43.13 \%$ & $43.50 \%$ & $45.67 \%$ \\
BH4 & T4 & $57.68 \%$ & - & $57.68 \%$ & $67.14 \%$ & $48.21 \%$ \\
SI2 & T4 and T3 & $71.70 \%$ & $72.50 \%$ & $70.89 \%$ & $68.75 \%$ & $74.64 \%$ \\
SI3 & T5 & $65.18 \%$ & $65.18 \%$ & - & $51.07 \%$ & $79.29 \%$ \\
SI4 & T6 and T2 & $58.39 \%$ & $56.92 \%$ & $59.67 \%$ & $55.36 \%$ & $61.43 \%$ \\
\hline
\end{tabular}

Table 2: Fraction of best choices in each block.

in choosing the best alternative from time series in BH2, SI2 and SI3, where autocorrelations are significant. The fraction of best choices is lowest with the highly unpredictable time series from WN2 and BH3. ${ }^{21}$ The numbers in columns four and five of Table 2 are similar. Thus, consistently with Result 1, experience with another time series does not improve performance.

Result 1 allows us to pool experienced and inexperienced participants for the remainder of the analysis. However, experience with a particular time series within a block may still be important. The last two columns of Table 2 show performance of participants (where experienced and inexperienced participants are pooled) in the first 20 and the last 20 periods of a block, respectively. ${ }^{22}$ For the time series from BH2, SI2, SI3, SI4 and, to a lesser extent, BH3 we see an overall increase in the fraction of optimal choices. ${ }^{23}$ This suggests that experience with a time series does have some effect on performance of the participants. Moreover, since one of our objectives is to investigate the effect of the characteristics of the time series on choice behavior (see Hypothesis 3 from Section 3.4) we believe that we should give the participants sufficient opportunities to get acquainted with the time series first. For the estimation of the discrete choice models in Sections 5 and 6 we therefore allow for a 'learning phase' of 20 periods and focus on describing choice behavior of the last 20 periods of each block.

\subsection{Return chasing}

We now investigate whether participants in the experiment have a tendency to 'chase returns', that is, whether they typically choose that alternative that had the highest actual return in the previous period. First, we analyze whether return chasing is a sensible strategy. Fig. 6 gives,

\footnotetext{
${ }^{21}$ Since in some blocks there were more than two alternatives, the fraction of best choices may not be the optimal measure to compare different blocks. Appendix E introduces an alternative efficiency measure. The comparisons on the basis of that measure give results consistent with the straightforward analysis here.

${ }^{22}$ Fig. 10 in Appendix E shows how the fractions of best choices evolved over time.

${ }^{23}$ There is a negative trend for the time series from WN2 and BH4. In both cases, a decrease in the fraction of best choices can be attributed to an unexpectedly high value of this fraction in the first 20 periods. This may occur because of the particular characteristics of the times series. In the WN2 blocks alternative A was the best relatively often in the first half of the experiment. Similarly, in the BH4 block, alternative C outperformed the other alternatives in each of the first thirteen periods, whereas there was not a clearly superior alternative in the remainder of that time series.
} 


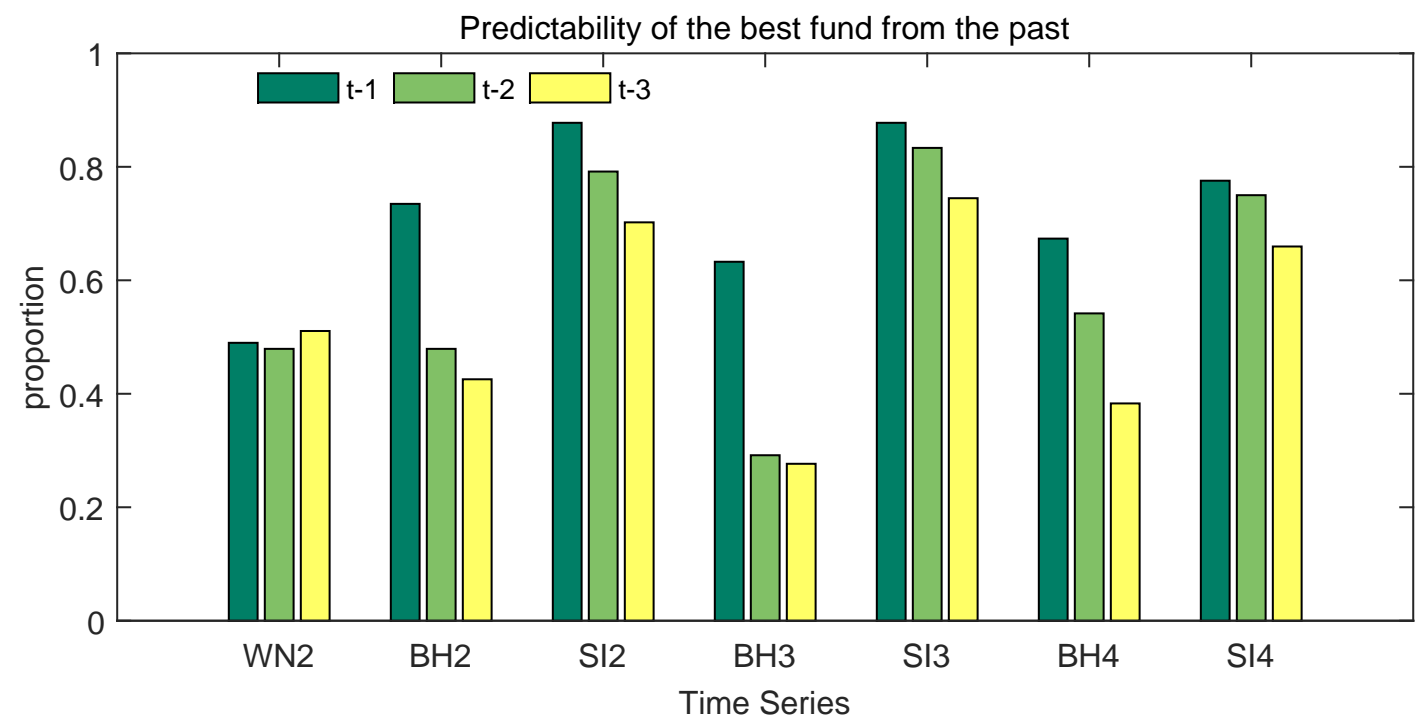

Figure 6: The proportion of periods when the optimal choice at time $t$ coincides with the optimal choice in period $t-1$, period $t-2$, and period $t-3$.
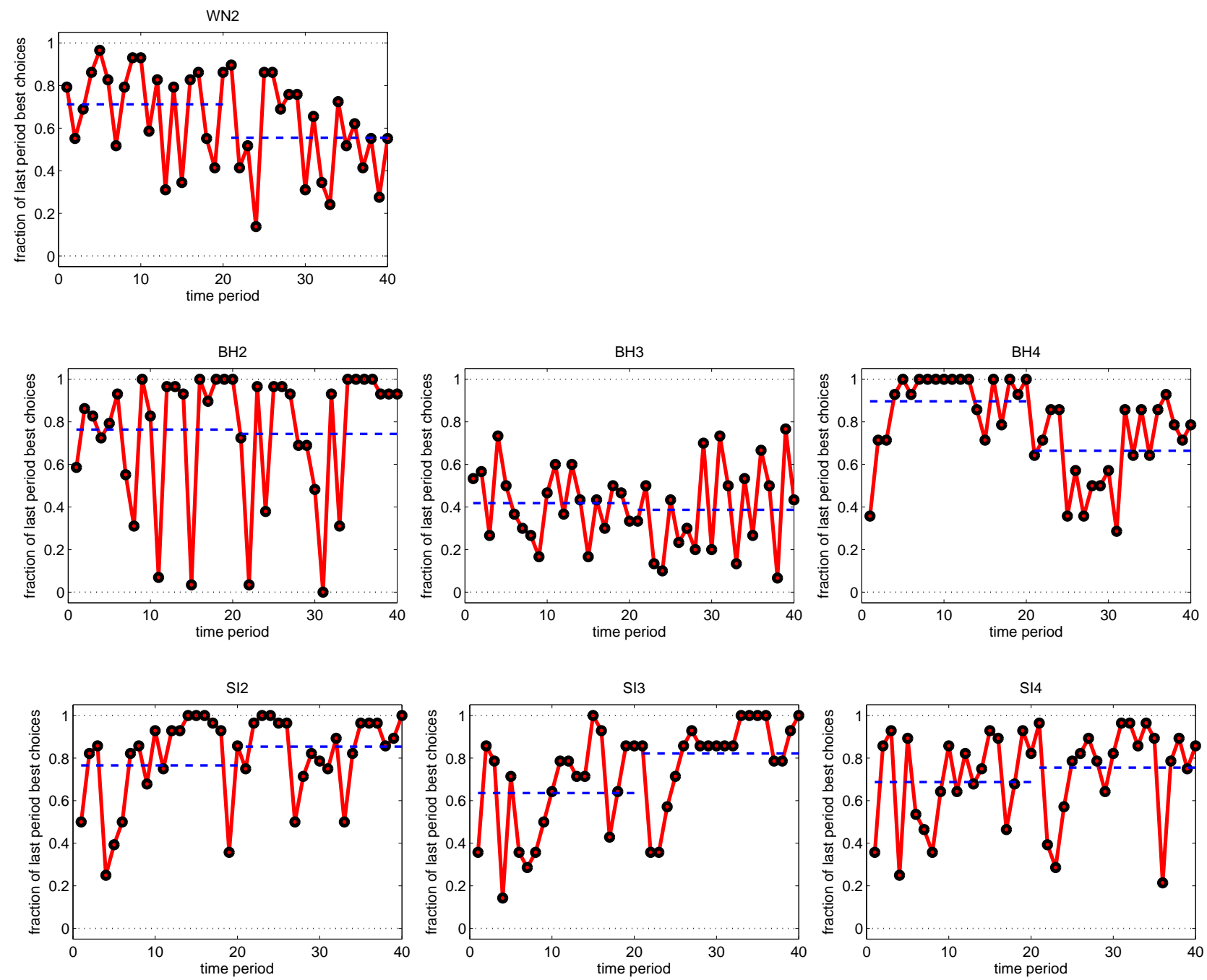

Figure 7: The fraction of participants choosing in period $t$ the fund that had highest returns in period $t-1$. Inexperienced and experienced sessions are pooled together. The horizontal dashed lines show the averages of the fractions over the first 20 and last 20 periods. 
for each set of time series, the proportion of periods for which the best alternative in period $t$ coincides with the best alternative in period $t-1, t-2$ and $t-3$, respectively. It follows that return chasing works best for the SI2 and SI3 sets of time series, for which it leads to the highest return in more than $80 \%$ of the periods. It also works relatively well in $\mathrm{BH} 2$, $\mathrm{BH} 4$ and SI4. ${ }^{24}$ Obviously, return chasing does not help in WN2. Also in the BH3 blocks its success would be relatively limited.

Fig. 7 provides evidence of return chasing by participants to the experiment. It plots the fraction of participants choosing the alternative in the current period that had the highest returns in the previous period. The more participants are chasing past returns, the closer the fractions in Fig. 7 are to 1. Return chasing is prevalent in BH2, BH4, SI2, SI3 and SI4 where participants choose the best alternative from the previous period around $80 \%$ of the time. Return chasing increases over time in the SI2, SI3 and SI4 blocks, but decreases substantially in the BH4 block. Remarkably, also in the WN2 blocks participants start with chasing returns, but over time learn to abandon that strategy. Only in the BH3 blocks return chasing seems to be relatively rare.

These observations suggest that a model where participants condition their decision upon the returns of the different alternatives from the previous period might organize the experimental data quite well. In the next two sections we estimate such models.

\section{Explaining choices in WN2, BH2 and SI2}

In this and the next section we investigate the extent to which the discrete choice models (1) and (2), discussed in Section 2, can explain the choice behavior of the participants in our experiment. We will estimate the models on the aggregate data using the method of maximum likelihood and discriminate between these models by taking advantage of the nested structure of the various specifications. We are interested in finding a parsimonious model that describes the data reasonably well. Moreover, due to their pivotal role in heterogeneous agent modeling, we have a specific interest in the estimated values of the Intensity of Choice parameters, such as $\beta$ in the basic model (1).

In this section we restrict attention to the binary choice model, using experimental data from those blocks of the experiment where the set of time series contains only two alternatives (i.e., WN2, BH2 and SI2). We start with estimating the one-lag model (model (1)) and turn to the multi-lag model (model (2)) in Section 5.2. In Section 6 we estimate the multiple choice model using the experimental data from the blocks with three and four alternatives.

To estimate the models we pool the data from experienced and inexperienced participants, which is justified by Result 1 . Also, to give participants the opportunity to get acquainted with

\footnotetext{
${ }^{24}$ Note that with two (four) alternatives, and a completely random time series, return chasing leads to the best alternative around $50 \%(25 \%)$ of the time.
} 
their economic environment, we will estimate our models on data from the last 20 periods of each block. More details, including our methodology and details of the goodness-of-fit tests, are reported in Appendix F.

\subsection{Binary choice models with a single lag}

Motivated by the qualitative evidence on return chasing behavior discussed in Section 4.3 we will try to explain the fraction of participants choosing alternative $\mathrm{A}$ as a function of the past returns of alternatives $\mathrm{A}$ and $\mathrm{B}$, for each of the three sets of time series with two alternatives.

Let us assume that the fraction of participants choosing alternative $\mathrm{A}$ at time $t$ is given as

$$
n_{t}^{A}=\frac{\exp \left[\alpha+\beta_{A} \pi_{A, t-1}\right]}{\exp \left[\alpha+\beta_{A} \pi_{A, t-1}\right]+\exp \left[\beta_{B} \pi_{B, t-1}\right]} .
$$

Note that only a constant for alternative A is included. ${ }^{25}$ As discussed in Section 2, parameter $\alpha$ represents a predisposition or bias towards one of the alternatives (see also Branch, 2004): if $\alpha$ is positive (negative) the participant tends to choose alternative A (B) even if returns in the previous period were the same. ${ }^{26}$ Eq. (4) allows for only one lag in past returns of the two alternatives, but the responses to these past returns may be asymmetric, $\beta_{A} \neq \beta_{B}$. Note that the vast majority of heterogeneous agent models use model (4) with $\alpha=0$ and $\beta_{A}=\beta_{B}$.

In finding a parsimonious model that gives a satisfactory description of participants' choices, we estimated equation (4) with three different specifications, using the method of maximum likelihood:

- PreAsym1: This is the original model in equation (4), which contains a predisposition effect and asymmetric responses to past returns.

- PreSym1: This corresponds to equation (4), with the restriction $\beta_{A}=\beta_{B}$ imposed. This model still has a predisposition effect, but symmetric responses to past returns.

- Sym1: This gives the specification with the restrictions $\beta_{A}=\beta_{B}$ and $\alpha=0$ imposed. This model corresponds to the canonical discrete choice model (1) for two alternatives.

We test the restrictions in PreAsym1 that lead to PreSym1 and Sym1 by the Likelihood Ratio (LR) test, as well as by comparing the information criteria (Akaike, AIC, and Bayesian, BIC) that penalize the models for additional parameters.

Table 3 presents the three estimated models, arranged from the most parsimonious model (Sym1) on top to the most general model (PreAsym1) at the bottom. We report the values

\footnotetext{
${ }^{25}$ This can be done without loss of generality (also see the discussion in Appendix F.1.1).

${ }^{26}$ Note that this interpretation assumes symmetric responses, $\beta_{A}=\beta_{B}$. For asymmetric responses the actual bias towards alternative $\mathrm{A}$ will also depend on $\beta_{A}, \beta_{B}$ and the average values of $\pi_{A}$ and $\pi_{B}$; see footnote 28 .
} 


\begin{tabular}{|c|c|c|c|c|c|c|c|c|c|}
\hline Model & Treatment & $\alpha$ & $\beta_{A}$ & $\beta_{B}$ & log-likelihood & AIC & BIC & $\begin{array}{l}\text { McFadden } \\
\text { R-squared }\end{array}$ & LR test \\
\hline \multirow{3}{*}{ Sym1 } & WN2 & 0.000 & $\begin{array}{c}0.100 \\
(0.030)\end{array}$ & 0.100 & -396.432 & 794.863 & 799.226 & -0.061 & \\
\hline & $\mathrm{BH} 2$ & 0.000 & $\begin{array}{c}0.431 \\
(0.034)\end{array}$ & 0.431 & -285.560 & 573.121 & 577.484 & 0.210 & \\
\hline & $\mathrm{SI} 2$ & 0.000 & $\begin{array}{c}3.747 \\
(0.277)\end{array}$ & 3.747 & -203.347 & 408.694 & 413.022 & 0.475 & \\
\hline \multirow{3}{*}{ PreSym1 } & WN2 & $\begin{array}{c}0.693 \\
(0.090)\end{array}$ & $\begin{array}{c}0.133 \\
(0.032)\end{array}$ & 0.133 & -364.927 & 733.854 & 742.580 & 0.023 & $\begin{array}{l}63.009 \\
(0.000)\end{array}$ \\
\hline & $\mathrm{BH} 2$ & $\begin{array}{l}-0.437 \\
(0.114)\end{array}$ & $\begin{array}{c}0.365 \\
(0.035)\end{array}$ & 0.365 & -278.606 & 561.212 & 569.938 & 0.230 & $\begin{array}{l}13.908 \\
(0.000)\end{array}$ \\
\hline & SI2 & $\begin{array}{c}0.626 \\
(0.162)\end{array}$ & $\begin{array}{c}4.409 \\
(0.370)\end{array}$ & 4.409 & -194.622 & 393.245 & 401.901 & 0.498 & $\begin{array}{l}17.449 \\
(0.000)\end{array}$ \\
\hline \multirow{3}{*}{ PreAsym1 } & WN2 & $\begin{array}{c}0.532 \\
(0.350)\end{array}$ & $\begin{array}{c}0.151 \\
(0.050)\end{array}$ & $\begin{array}{c}0.123 \\
(0.038)\end{array}$ & -364.813 & 735.626 & 748.715 & 0.024 & $\begin{array}{c}0.228 \\
(0.633)\end{array}$ \\
\hline & $\mathrm{BH} 2$ & $\begin{array}{c}-12.973 \\
(1.516)\end{array}$ & $\begin{array}{c}2.609 \\
(0.299)\end{array}$ & $\begin{array}{c}0.095 \\
(0.051)\end{array}$ & -223.469 & 452.938 & 466.027 & 0.382 & $\begin{array}{c}110.274 \\
(0.000)\end{array}$ \\
\hline & $\mathrm{SI} 2$ & $\begin{array}{c}0.925 \\
(0.374)\end{array}$ & $\begin{array}{c}4.652 \\
(0.471)\end{array}$ & $\begin{array}{c}4.754 \\
(0.548)\end{array}$ & -194.221 & 394.442 & 407.426 & 0.499 & $\begin{array}{c}0.803 \\
(0.370)\end{array}$ \\
\hline
\end{tabular}

Table 3: Comparison of the three models with one lag over all treatments. Last 20 periods of data are used (pooled over experiences). The last column shows the results of LR test of the corresponding model against the previous (restricted) model.

of the parameters, the standard deviations (in parentheses), the values of the log-likelihood function and the information criteria. For each set of time series, the value of the information criterion for the best model (i.e., with the lowest AIC or BIC value) is shown in bold. Table 3 also shows McFadden's $\mathrm{R}^{2}$ that evaluates the goodness of fit of a model by comparing it with the model where only the constant term is included (the latter model corresponds to a "pure predisposition' model). The last column of Table 3 should be read from down to up. It shows, for the corresponding set of time series and estimated model, the value of the log-likelihood ratio and the $p$-value at the $5 \%$ significance level (in parentheses) when the model is tested against its restricted version from the row immediately above.

Thus, for the WN2 blocks we test (i) the hypothesis that $\beta_{A}=\beta_{B}$ in the PreAsym1 model, leading to the PreSym1 model (the LR is 0.228 and we cannot reject the hypothesis), and (ii) the hypothesis that $\alpha=0$ in the PreSym1 model leading to the Sym1 model (the LR is 63.009 and we therefore have to reject this hypothesis). This suggests that the PreSym1 model is the most parsimonious model that gives a good description of the aggregate data in the WN2 blocks. The information criteria support this conclusion. In the same way (and also consistent with the AIC and the BIC) we conclude that the PreSym1 model is the best one-lag model to describe the data from the SI2 blocks. In contrast, the best model describing the BH2 blocks is PreAsym1 with asymmetric responses in addition to a predisposition effect. We summarize these and subsequent results regarding the optimal models (as selected by the LR test) in the second and third column of Table 4 in Section 7.

The left panels in Fig. 8 compare the share of participants choosing alternative $\mathrm{A}$ in the experiment (thick solid line) with the predicted fraction of participants choosing alternative A using the estimated PreSym1 model from Table 3 (thick dashed line) and, for the BH2 time series, also with the predictions of the PreAsym1 model (thin solid line). The panels illustrate that the PreSym1 model describes the data from the WN2 and SI2 blocks quite well. In fact, 
also for the data from the BH2 blocks model PreSym1 gives a fairly good description although model PreAsym1, with asymmetric responses to returns, performs significantly better.

It follows from the results presented in Table 3, that the estimated value of the intensity of choice parameter $\beta$ is significantly positive at the $5 \%$ level for the selected model PreSym1 in the WN2 and SI2 blocks. This is particularly noteworthy for the WN2 blocks since past returns in those blocks have no predictive power for future returns. Also for the $\mathrm{BH} 2 \mathrm{blocks}$, the estimated value of $\beta_{A}$ in the selected model PreAsym1 is significantly positive at the $5 \%$ level. ${ }^{27}$ Our result therefore is the following.

Result 2. We confirm Hypothesis 2 and conclude that human subjects do exhibit 'return chasing behavior' in the WN2, BH2 and SI2 blocks.

As discussed in Section 2 this result is important for the literature on heterogeneous agent models, because such 'return chasing' behavior (defined over performances of various behavioral rules) is a crucial assumption in these models. Experimental evidence of validity of this assumption is therefore reassuring. However, at the same time our results show that the canonical specification (1) (equivalent to model Sym1) used in the vast majority of the theoretical and computational contributions to this literature does not provide a satisfactory description of aggregate choice behavior for any of the three sets of time series considered.

When we analyze the estimated models from Table 3 in a bit more detail we can make two additional observations. First, the predisposition effect is significantly different from 0 for all three sets of time series, and implies a bias towards alternative A. ${ }^{28}$ A possible explanation for this is a simple preference of participants to choose the first presented option. Closer inspection

\footnotetext{
${ }^{27}$ In that model, however, $\beta_{B}$ is not significantly different from 0 . This means that participants respond to the payoffs for alternative A, but not so much to payoffs for alternative B. Considering the time series of these alternatives, shown in upper left panel of Fig. 3, we see that for most periods alternative A has a lower payoff (around 4.50) than alternative B (which in many periods gives a payoff of around 7.50). Moreover, an increase in the returns for alternative A occurs simultaneously with a decrease in the return for alternative B. The estimated PreAsym1 model from Table 3 suggests that the default option for the participants is to choose alternative B, except if payoffs for alternative $\mathrm{A}$ in the previous period were substantially higher than their lower bound of 4.5. That is, the estimated model can be written as

$$
n_{t}^{A}=\frac{1}{1+\exp \left(-2.608\left(\pi_{A, t-1}-4.97\right)\right)}
$$

meaning that for $\pi_{A, t-1}=4.50$ (which is around the value that $\pi_{A}$ takes on most often), the fraction of the participants choosing $\mathrm{A}$ is approximately 0.22 , whereas $n_{A, t}$ will be above $0.50(0.90)$ if $\pi_{A, t-1}$ is above 5 (6).

${ }^{28}$ To see this, one can substitute, for the three sets of time series, the average payoffs for alternatives A and $\mathrm{B}$ (as given in Table 5) in the estimated model from Table 3 that gives the best description of the data and compute the predicted fraction of participants choosing A. This gives $n^{*} \approx 0.68$ for $\mathrm{WN} 2, n^{*} \approx 0.53$ for $\mathrm{BH} 2$ and $n^{*} \approx 0.93$ for SI2. Note that although the estimate of $\alpha$ is negative in $\mathrm{BH} 2$, the asymmetry in responses to payoffs is large enough to lead to a bias towards A.
} 

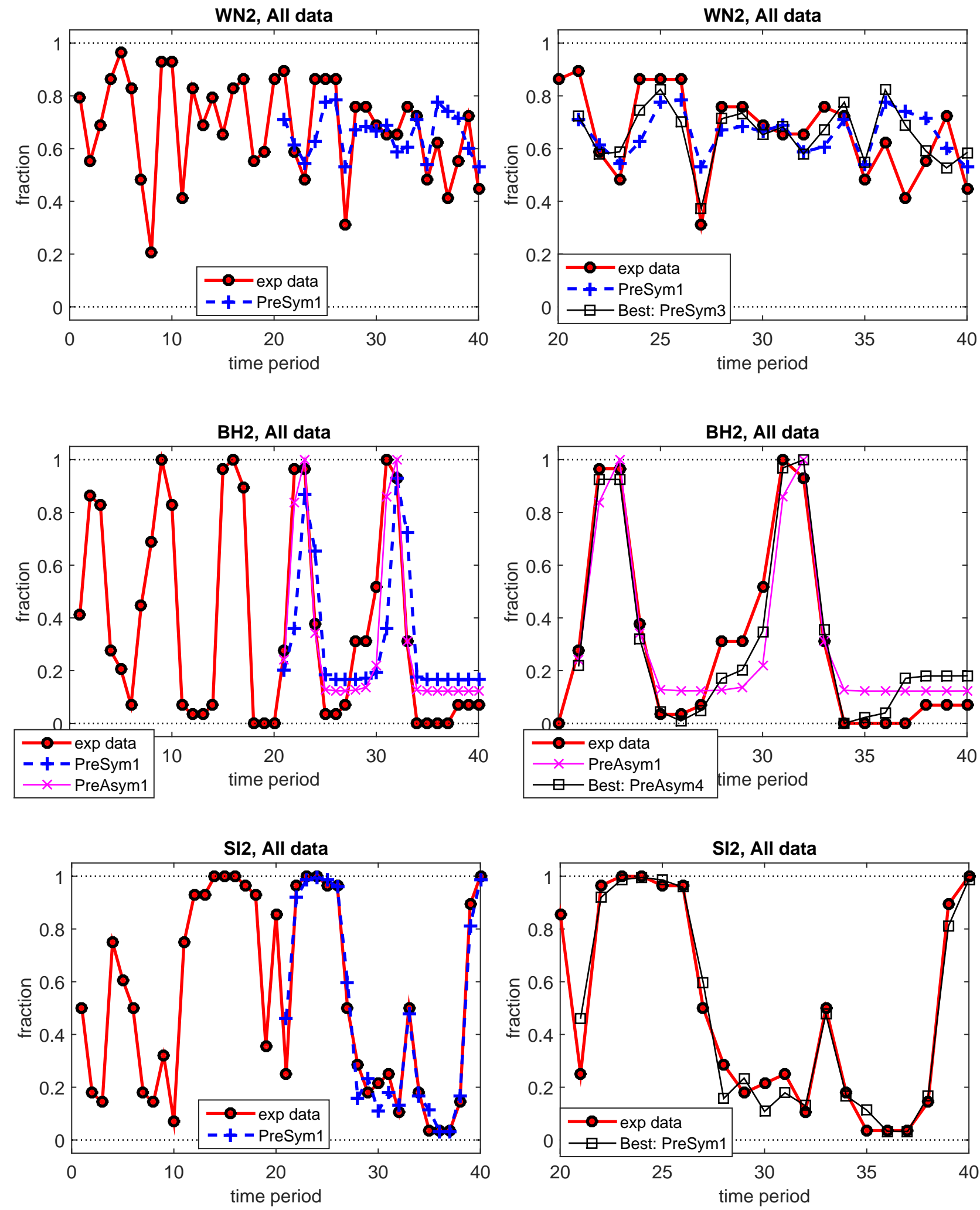

Figure 8: Experimental data (thick solid line with circles) and model predictions. Left panels: All 40 periods are shown; predictions are from the one-lag models, PreSym1 and PreAsym1 (only for BH2). Right panels: the 20 last periods are shown; predictions are from multiple lags model compared with the best prediction from the one-lag model. The best model is shown by the thin line with squares. 
of the different time series may provide sensible alternative explanations as well. ${ }^{29}$ In the time series from WN2, for example, by chance A gives a higher payoff than B in 7 out of the first 10 periods $(t=1, \ldots, 10)$. For the SI2 blocks, on the other hand, returns for these 10 periods are roughly the same for choices $\mathrm{A}$ and $\mathrm{B}$, but in the starting periods $(t=-9, \ldots, 0)$ alternative $\mathrm{A}$ is dominating alternative $\mathrm{B}$ by a substantial margin. These initial differences may explain the existence of the predisposition effect, even allowing for a learning phase of 20 periods. ${ }^{30}$

Second, we see that the estimated intensity of choice parameter $\beta$ differs for different sets of time series. As the return for all alternatives in each block is on average about 5 points per period (see Table 5 in Appendix B), the differences between the estimated intensities of choice can not be attributed to a scaling effect. We find the estimated $\beta$ to be quite high $(\beta \approx 4.4)$ for the SI2 blocks, and low $(\beta \approx 0.1)$ for the WN2 blocks. This ordering is consistent both with Fig. 6, which shows persistence of the best fund over time for different blocks, and with the autocorrelation structure in returns. Choosing the alternative that generated a high return in the previous period makes sense in particular when returns are highly and positively autocorrelated. The results suggest that the intensity of choice is affected by the structure of the time series of returns, and participants are successful in adjusting their reaction to past information in the right direction (e.g., their behavior is described by a higher value of $\beta$ when past returns have more predictive power about future returns). Based on this observation we conclude the following:

Result 3. We reject Hypothesis 3. The estimated value of the intensity of choice parameter $\beta$ depends strongly on the predictability of returns. In particular, $\beta$ is larger when the autocorrelation structure in the returns is stronger (and positive).

\subsection{Binary choice models with multiple lags}

Result 3 suggests that predictability of future returns is an important determinant of the estimated intensity of choice parameter $\beta$, which is higher the higher the autocorrelation in the time series of returns is. It therefore makes sense to consider an extended model that includes more lags of past returns to allow for the possibility that participants try to exploit the structure

\footnotetext{
${ }^{29}$ It is difficult to explain the predisposition effect by risk aversion. To see this, note from Table 5 from AppendixB that the differences in variances in returns is substantial for the choice sets BH2 and SI2, whereas the difference in means is small. Participants' risk aversion should then be represented by a predisposition towards the alternative with the lower variance. Comparing Table 5 with Table 3 we see that this is the case for the BH2 set of time series but not for the SI2 set, where there is a predisposition towards the alternative with the higher variance.

${ }^{30}$ To illustrate that the predisposition effect diminishes somewhat over time we also estimated model PreSym1 for WN2 on all 40 periods. Recomputing, for this estimated model, the predicted fraction for A when payoffs for the alternatives are equal to their mean values, we find $n^{*} \approx 0.73$ (cf. footnote 28 ).
} 
in the time series. That is, we consider specification (2), which for two alternatives becomes:

$$
n_{t}^{A}=\frac{\exp \left[\alpha+\sum_{\ell=1}^{L} \beta_{A, \ell} \pi_{A, t-\ell}\right]}{\exp \left[\alpha+\sum_{\ell=1}^{L} \beta_{A, \ell} \pi_{A, t-\ell}\right]+\exp \left[\sum_{\ell=1}^{L} \beta_{B, \ell} \pi_{B, t-\ell}\right]} .
$$

Note that we allow for a predisposition effect and for differences between the coefficients on past returns (both across time and across alternatives), but we do assume that participants consider the same number of lags $L$ for each alternative. ${ }^{31}$

We use the following methodology for estimating model (5). We begin with estimating the model with only a constant, i.e., with $L=0$. We then add one lag for both alternatives, estimate this less parsimonious model and test (using the LR test with a significance level of $5 \%$ ) the restriction $\beta_{A, 1}=\beta_{B, 1}=0$, which leads to the first model. If this restriction is rejected we reject the first model (with $L=0$ ) and proceed in the same way with the second model. That is, we add a second lag for both alternatives, estimate this new model and test if this model is significantly better than the previous one, and so on. The procedure stops as soon as adding a lag for both alternatives does not significantly improve the performance of the model. We then choose the model which was the last one that provided a significant improvement. ${ }^{32}$ Using this procedure we end up with $L=3$ for the WN2 set of time series (we call the resulting model PreAsym3), with $L=4$ for the BH2 set of time series (the PreAsym4 model) and with $L=1$ for the SI2 set of time series (the PreAsym1 model). The rest of our estimation strategy is the same as in the previous section. Namely, we test the hypothesis that coefficients on past returns are the same for both alternatives, that is $\beta_{A, \ell}=\beta_{B, \ell}$ for all $\ell \leq L$, and then, if this hypothesis cannot be rejected with the LR test, we attempt to simplify the model further by testing the additional restriction, $\alpha=0$. Tables 6 and 7 in Appendix C.1 provide the results of this final part of the estimation procedure, for the WN2 and BH2 blocks, respectively. For the SI2 block, the results coincide with those in Table 3, i.e., model PreSym1 is selected.

For the WN2 block, after selecting model PreAsym3 in the first step of the estimation procedure, we cannot reject the hypothesis that $\beta_{A, \ell}=\beta_{B, \ell}$ for all $\ell \leq 3$ but, we do reject the restriction $\alpha=0$ and therefore arrive at the PreSym3 model. However, comparing the BIC values for the estimated multi-lag models from Table 6 in Appendix C.1 and the estimated onelag model in Table 3, we find that the more parsimonious model PreSym1 is almost as good as the PreSym3 model. This suggests that, for the WN2 time series, the one-lag PreSym1 model is sufficiently flexible to provide a good explanation of the data.

For the BH2 blocks, the PreAsym4 model selected in the first step of the estimation procedure cannot be simplified any further (the restriction $\beta_{A, \ell}=\beta_{B, \ell}$ for all $\ell \leq 4$ is rejected

\footnotetext{
${ }^{31}$ See Appendix F.3 for a formal specification of the model with lags. We also estimated the models which include an arbitrary number $L_{A}<10$ of lags for alternative A and an arbitrary number $L_{B}<10$ of lags for alternative B. The results are available upon request.

${ }^{32} \mathrm{An}$ alternative procedure is to start with, for example, $L=10$ lags and then sequentially reduce the number of lags as long as the hypothesis that the two coefficients on the highest lag are equal to zero is not rejected. In general this procedure may lead to different results, e.g., with a higher number of lags in the final model.
} 
by the LR test). For the $\mathrm{BH} 2$ blocks there is a substantial improvement in considering a discrete choice model with more than one lag in returns (as can be seen by comparing the AIC and BIC values for this model in Table 7 with those for the PreAsym1 model in Table 3). The estimated coefficients of the PreAsym4 model, as reported in Table 7, are remarkably high, with the first and third coefficient significantly different from zero but with opposite signs. This suggests that participants try to pick up the cyclic behavior in the return differences. Although they respond positively to last periods return difference, they correct this with a negative response to the return difference of two periods before.

The right panels of Fig. 8 compare, for the last 20 periods, the share of participants in the experiment choosing alternative A, the predicted share by the selected model with one lag as described in Section 5.1 (see Table 3), and the predicted share by the chosen multi-lag model as described in this Section (see Tables 6 and 7 in Appendix C.1). The estimated multi-lag model clearly outperforms the one-lag model for the data in the BH2 blocks. For the SI2 blocks the best multi-lag model is in fact the model with one lag, and for the WN2 blocks performance of the two models is similar.

These findings suggest that the structure in the time series is an important determinant of the choice behavior of experimental participants. When there is either very limited structure (as in the WN2 blocks), or very salient structure (as in the SI2 blocks), participants tend to condition their choice only upon the most recent information and a discrete choice model with one lag seems sufficient to describe the data. When the structure is more complicated (e.g., when it has a cyclical pattern as in the time series from the BH2 blocks), participants seem to condition their behavior on a longer history of returns. We therefore have the following

Result 4. Hypothesis 4 is rejected. In particular for the BH2 block, the multi-lag model gives a substantially better, and qualitatively different, description of the participants' decisions.

To summarize, we find that the discrete choice model is successful in explaining the experimental data from the WN2, BH2 and SI2 blocks, taking into account the following qualifications. First, a predisposition effect in the discrete choice model is warranted, as this effect is significant for all three models that performed best in describing the data (the PreSym1 model for the WN2 and SI2 blocks and the PreAsym4 model for the BH2 blocks). Second, although for models with either a little or a lot of structure in the time series a discrete choice model with one lag and symmetric responses performs well, for time series with a nontrivial structure more lags and asymmetric responses may be required. Importantly, the intensity of choice parameter increases with the predictability in the time series. Finally, note that the discrete choice model that is typically used in the literature, model Sym1, does not do a very good job in explaining the experimental data. ${ }^{33}$

\footnotetext{
${ }^{33}$ Some extensions of the discrete choice model (Hommes et al., 2012; Anufriev and Hommes, 2012b) explicitly allow for more payoff lags in the performance measure, introducing so-called "memory" with geometrically declining weights. That is, the weight of payoff $\pi_{t-\tau}$ is given by $\beta \eta^{\tau-1}$, with $\eta \in(0,1)$. Inspection of Tables 6 and 7 suggests that such a model would not describe the behavior of participants in this experiment very well, since the estimated coefficients are clearly not geometrically decreasing.
} 


\section{Explaining choices in $\mathrm{BH} 3, \mathrm{BH} 4, \mathrm{SI} 3$ and SI4}

In this section we briefly discuss estimation results for a general discrete choice or multinomial logit model with one lag, applied to the experimental data from the blocks with three or four alternatives (that is, the BH3, BH4, SI3 and SI4 blocks). An important feature of the multinomial logit model with more than two alternatives is that it is more flexible than the binary choice model. In the case of three alternatives, for example, the general model assumes the following proportionalities of the fractions of choices for alternatives $\mathrm{A}, \mathrm{B}$ and $\mathrm{C}$ as functions of past performances:

$$
\begin{aligned}
& n_{t}^{A} \propto \exp \left[\alpha^{A}+\beta_{A}^{A} \pi_{A, t-1}+\beta_{B}^{A} \pi_{B, t-1}+\beta_{C}^{A} \pi_{C, t-1}\right], \\
& n_{t}^{B} \propto \exp \left[\alpha^{B}+\beta_{A}^{B} \pi_{A, t-1}+\beta_{B}^{B} \pi_{B, t-1}+\beta_{C}^{B} \pi_{C, t-1}\right], \\
& n_{t}^{C} \propto \exp \left[\alpha^{C}+\beta_{A}^{C} \pi_{A, t-1}+\beta_{B}^{C} \pi_{B, t-1}+\beta_{C}^{C} \pi_{C, t-1}\right] .
\end{aligned}
$$

Note that the fraction choosing an alternative may be directly affected by the past returns of any of the other alternatives, whereas this is not the case for the binary choice model. ${ }^{34}$ Another important characteristic is that, although model (6) has twelve parameters, only eight of them can be identified. To see this, note that, for example, subtracting $\beta_{A}^{C} \pi_{A, t-1}$ from each of the profit sums in (6) gives rise to exactly the same normalized fractions. Selecting alternative $\mathrm{C}$ as the 'reference option', we can then write the three normalized fractions as

$$
\begin{aligned}
& n_{t}^{A}=\exp \left[\left(\alpha^{A}-\alpha^{C}\right)+\left(\beta_{A}^{A}-\beta_{A}^{C}\right) \pi_{A, t-1}+\left(\beta_{B}^{A}-\beta_{B}^{C}\right) \pi_{B, t-1}+\left(\beta_{C}^{A}-\beta_{C}^{C}\right) \pi_{C, t-1}\right] / Z, \\
& \left.n_{t}^{B}=\exp \left[\left(\alpha^{B}-\alpha^{C}\right)+\left(\beta_{A}^{B}-\beta_{A}^{C}\right) \pi_{A, t-1}+\left(\beta_{B}^{B}-\beta_{B}^{C}\right) \pi_{B, t-1}+\left(\beta_{C}^{B}-\beta_{C}^{C}\right) \pi_{C, t-1}\right]\right] / Z, \\
& n_{t}^{C}=1 / Z,
\end{aligned}
$$

where $Z$ is a normalization factor which is chosen in such a way that $n_{t}^{A}+n_{t}^{B}+n_{t}^{C}=1$.

We refer to (7) as the General1 model. This model has eight parameters (two relative predisposition effects, $\alpha^{h}-\alpha^{C}$ for $h=A, B$, and six relative intensities of choice of the form $\beta_{k}^{h}-$ $\beta_{k}^{C}$ for $h=A, B$ and $\left.k=A, B, C\right)$. This General1 model nests the PreAsym1, PreSym1 and Sym1 models that were discussed (for two alternatives) in Section 5. In particular, imposing the restriction that $\beta_{k}^{h}=\beta_{k}^{g}$ for all $k, h$ and $g$ that are different from each other, gives the PreAsym1 model

$$
n_{t}^{h}=\frac{\exp \left[\alpha_{h}+\beta_{h} \pi_{h, t-1}\right]}{\exp \left[\alpha_{A}+\beta_{A} \pi_{A, t-1}\right]+\exp \left[\alpha_{B}+\beta_{B} \pi_{B, t-1}\right]+\exp \left[\beta_{C} \pi_{C, t-1}\right]} \quad \text { for } h=A, B, C
$$

\footnotetext{
${ }^{34}$ The general form implied by (6) reduces to the PreAsym1 model from Section 5.1 if there are only two alternatives, see Appendix F for details. Since the fractions need to be normalized to make them add up to one, the fractions in the binary choice PreAsym1 model are indirectly affected by payoffs of the other alternatives. But in the case with more than two alternatives, the dependence is also direct and more complicated.
} 
which has five parameters (note that $\left.\alpha_{C}=0\right) .{ }^{35}$ Imposing in addition that the three intensities of choice are the same $\left(\beta_{A}=\beta_{B}=\beta_{C}=\beta\right)$ we obtain the PreSym1 model. Finally, the oneparameter canonical Sym1 model is obtained when requiring that there are no predisposition effects $\left(\alpha_{A}=\alpha_{B}=0\right)$, which gives model (1) from Section 2 with $H=3$. For the case of four alternatives the different models and restrictions are similar (see Appendix F for details). ${ }^{36}$

Given this general framework we search for a multinomial logit model that fits the experimental data from the BH3, BH4, SI3 and SI4 blocks. We apply the same methodology as in Section 5.1. That is, we start with estimating the General1 model and then investigate, using the LR test and information criteria, whether a more parsimonious model exists that still gives a good description of the experimental data. Note that we have now one additional step, from the General1 to the PreAsym1 model. This step is interesting, because the literature on heterogeneous agent models assumes that there is no direct effect of other alternatives (i.e., it imposes restriction $\beta_{H}^{K}=0$ for $H \neq K$ in (6)) and thus never considers the General1 model.

Further details and the estimation results are presented in Appendix C.2. Below we give a brief overview of the main findings, which are summarized in Table 4 in Section 7.

\subsection{Choices in SI3 and SI4}

The estimation results for the SI3 and SI4 blocks are presented in Tables 8 and 9 in Appendix C.2, respectively. Applying the results of the LR tests at the $5 \%$ significance level to the models estimated on the experimental data from the SI3 block, we find that the PreSym1 model is the most parsimonious model that still gives a good description of the data. This is consistent with the BIC, which is indeed lowest for the PreSym1 model, but not with the AIC (which selects the PreAsym1 model, although the AIC values are quite close).

Note that the same specification (the PreSym1 model) was chosen for the SI2 blocks (recall that the time series for alternatives A and B are exactly the same in the SI2 and SI3 blocks). Moreover, the predisposition effect towards alternative A that we found for the SI2 blocks (see Table 3) is preserved when adding a third time series (from Table 8 we see that the estimated predisposition for alternative A, relative to the new alternative $\mathrm{C}$, is 0.688 , whereas the predisposition for alternative $\mathrm{B}$, again relative to alternative $\mathrm{C}$, is -1.014 ). The estimated intensity of choice, however, although still relatively high at $\beta \approx 1.97$, is about twice as small as the intensity of choice for the PreSym1 model estimated on the data from the SI2 blocks. Inspection of the time series in the upper left and middle left panels of Fig. 4 may help explain

\footnotetext{
${ }^{35}$ These five parameters are related to the parameters of the General1 model (7) as follows: the two (relative) predisposition effects are $\alpha_{A}=\alpha^{A}-\alpha^{C}$ and $\alpha_{B}=\alpha^{B}-\alpha^{C}$ and the three (relative) intensities of choice are given by $\beta_{A}=\beta_{A}^{A}-\beta_{A}^{C}, \beta_{B}=\beta_{B}^{B}-\beta_{B}^{C}$ and $-\beta_{C}=\beta_{C}^{A}-\beta_{C}^{C}$.

${ }^{36}$ If there are four alternatives, the General1 model has fifteen parameters; the PreAsym1 model has seven parameters (three relative predisposition effects and four intensities of choice); the PreSym1 model has four parameters; and the Sym1 model has one parameter, the intensity of choice $\beta$. The latter model was used in the case of four strategies discussed in Brock and Hommes (1998). Appendix F.2 provides formal equations, parameters to be estimated and restrictions.
} 
this. Although in periods 1 until about 10 returns for alternatives $\mathrm{A}$ and $\mathrm{B}$ are roughly the same, in the subsequent fifteen periods alternative A consistently outperforms alternative B. Participants that only need to choose between these two alternatives may therefore learn that the strategy of selecting the alternative that had the highest payoff in the previous period is very successful, which is reflected in a high value of the intensity of choice. The success of such a strategy is slightly inhibited, however, when adding alternative $\mathrm{C}$. This new alternative sometimes, but not always, outperforms alternative $\mathrm{A}$ in the first 25 periods. As a consequence the estimated intensity of choice for the SI3 block is smaller.

Remarkably, where the experimental data from both the SI2 and SI3 blocks give rise to the PreSym1 model, the chosen model for the data from the SI4 blocks, the General1 model, is considerably more complicated. Although not all estimated coefficients in the General1 model presented in the last row of Table 9 are significantly different from zero, the restrictions that would reduce that model to the PreAsym1 model are rejected (let alone simplifying it further to the PreSym1 model), a finding that is also consistent with the two information criteria. Note that the first three time series in the SI4 blocks are the same as in the SI3 block, and that only the time series corresponding to alternative D is added for the SI4 blocks. This new time series has a rather straightforward relation to the time series of the other three alternatives. In particular, as can be seen from the lower left panel of Fig. 4, it is the worst of the four alternatives in each of the periods 1 until 15, but the best alternative for almost all of the last fifteen periods. One would therefore not expect the addition of this alternative to complicate the decision problem of the participants substantially. A more reasonable explanation may be that participants are in general better able to make decisions between two or three alternatives than between four alternatives, which is consistent with the finding that a much more complex model structure is required to describe their behavior satisfactorily for this latter case. ${ }^{37}$

\subsection{Choices in $\mathrm{BH} 3$ and $\mathrm{BH} 4$}

We now consider the experimental data from the $\mathrm{BH} 3$ and $\mathrm{BH} 4$ blocks. Contrary to the SI blocks, there exists no relationship between the time series from the $\mathrm{BH} 2, \mathrm{BH} 3$ and $\mathrm{BH} 4$ blocks. These two sets of time series are special, however, in the sense that the different time series in a given block are not linearly independent. In particular, for the time series from the BH3 blocks we have that $\pi_{A, t}=\frac{1}{2}\left(\pi_{B, t}+\pi_{C, t}\right)$ for every $t$, and for the time series from the BH4 block we have $\pi_{A, t}=c_{B} \pi_{B, t}+c_{C} \pi_{C, t}+c_{D} \pi_{D, t}$ for every $t$, with $c_{B}=c_{C} \approx 2.881$ and $c_{D} \approx-4.762$. This linear dependence has important consequences for estimating the multinomial logit model. More specifically, it means that the parameters of the multinomial logit model are not identifiable. We address this problem by using (for the BH3 block) the linear dependence to modify the

\footnotetext{
${ }^{37}$ Note that the (relative) intensities of choice associated with the return for alternative D (see the last column of the General1 model in Table 9) are relatively high, suggesting that participants condition their behavior to a substantial extent on returns of that alternative. Also note that, although returns of one particular alternative may directly effect the fractions of all alternatives, the strongest effect is typically on the fraction of participants using that particular alternative (that is, typically $\beta_{h}^{h}>\beta_{k}^{k}>0$ for $k \neq h$ and $k, h \in\{A, B, C\}$ ).
} 
General1 model (7) as follows

$$
\begin{aligned}
& n_{t}^{A}=\exp \left[\left(\alpha^{A}-\alpha^{C}\right)+\left(\tilde{\beta}_{B}^{A}-\tilde{\beta}_{B}^{C}\right) \pi_{B, t-1}+\left(\tilde{\beta}_{C}^{A}-\tilde{\beta}_{C}^{C}\right) \pi_{C, t-1}\right] / Z, \\
& \left.n_{t}^{B}=\exp \left[\left(\alpha^{B}-\alpha^{C}\right)+\left(\tilde{\beta}_{B}^{B}-\tilde{\beta}_{B}^{C}\right) \pi_{B, t-1}+\left(\tilde{\beta}_{C}^{B}-\tilde{\beta}_{C}^{C}\right) \pi_{C, t-1}\right]\right] / Z, \\
& n_{t}^{C}=1 / Z
\end{aligned}
$$

where, for every alternative $h=A, B, C$, we define the modified (and identifiable) coefficients $\tilde{\beta}_{B}^{h}=\beta_{B}^{h}+\frac{1}{2} \beta_{A}^{h}$ and $\tilde{\beta}_{C}^{h}=\beta_{C}^{h}+\frac{1}{2} \beta_{A}^{h}$, and where $Z$ is required for normalization. Note that the interpretation of the parameters $\tilde{\beta}_{k}^{h}$ differs from that of $\beta_{k}^{h}$ (for $k=B, C$ ). A high value of the former can derive from the fact that participants are sensitive to returns generated by alternative $k$ or that they are sensitive to returns generated by alternative A (or by a combination of both). This makes interpretation of the estimated model more difficult. Appendix C.2 explains how the PreAsym1, the PreSym1 and the Sym1 models are defined in this case. Similarly, the models describing the data from the BH4 block become identifiable after defining for every fund $h=A, B, C, D$ the parameters $\tilde{\beta}_{B}^{h}=c_{B} \beta_{A}^{h}+\beta_{B}^{h}, \tilde{\beta}_{C}^{h}=c_{C} \beta_{A}^{h}+\beta_{C}^{h}$, and $\tilde{\beta}_{D}^{h}=c_{D} \beta_{A}^{h}+\beta_{D}^{h}$.

Table 10 in Appendix C.2 provides the estimation and test results for the data from the BH3 blocks. The parameter restrictions leading to the PreAsym1 model are rejected, but only weakly (the $p$-value is 0.048). Furthermore, for the PreAsym1 model the restrictions that lead to the PreSym1 model are not rejected and for the PreSym1 model the restrictions that lead to the Sym1 model are also not rejected. In addition, the two information criteria select the Sym1 model as the best model for the BH3 blocks. Overall, therefore, it seems that the Sym1 model gives a good description for participants' decisions in the BH3 blocks. Note that this is the only set of time series for which decisions are consistent with the canonical discrete choice model (1). ${ }^{38}$ Relevant in this respect might be that the BH3 time series are relatively unpredictable (see the middle left and right panels of Fig. 3 and also Fig. 6).

The results are, again, notably different when there are four alternatives, as can be seen from Table 11 in Appendix C.2. Here the two information criteria as well as the LR tests select the General1 model as the optimal model, which is consistent with the findings for the SI4 blocks. $^{39}$ These results suggest that more complex multinomial logit models are required to explain choice behavior when there are four or more alternatives.

The results in this section can be summarized as follows

Result 5. We reject Hypothesis 5. Experimental data from blocks with more than three alternatives can only be described satisfactorily by models that are substantially more complicated than the models required to describe the experimental data from blocks with two or three alternatives.

\footnotetext{
${ }^{38}$ The underlying data generating process used a Sym1 model as well.

${ }^{39}$ Note that the time series in the BH4 block seem to be (slightly) more predictable than those in the BH3 blocks (see Fig. 3), which is also consistent with the better performance of participants in the BH4 block as compared to the BH3 blocks (see Table 14). Again, therefore, it is hard to argue that the decision problem for the BH4 block is substantially more complicated than the decision problem for the BH3 blocks.
} 


\section{Discussion}

In this paper we presented a laboratory experiment where participants observe past performance of two, three, or four different investment alternatives (framed as financial funds) and choose between these alternatives for a number of consecutive periods. Participants switch regularly between alternatives and their behavior is driven, to a large extent, by the past returns of the different alternatives - in particular by the most recent returns. We also find that experience with a different set of time series has no discernible effect on participants' choices.

Our main goal is to analyze whether the well known discrete choice model can explain the behavior of participants. Because such framework plays a central role in heterogeneous agent models, evidence for its relevance in laboratory experiments would provide a sound empirical microfoundation for these models. Such a microfoundation has been lacking until now. We find that behavior of participants can be described by discrete choice models reasonably well. Table 4 summarizes our findings. It shows, for each set of time series that we use in the experiment, the version of the discrete choice model that gives the best description of the choices made by the participants. It also identifies how the estimated models differ from the canonical discrete choice model that is used in a large majority of heterogeneous agent models. The table, together with our analyses from Sections 5 and 6, provides guidelines for scholars working in the field of heterogeneous agent models which they can use when constructing their models. We list some of those guidelines below.

- The canonical discrete choice model (1) gives a good description of choice behavior only for the BH3 blocks. For all of the other sets of time series a predisposition towards one of the alternatives has to be included.

- A model with one lag and symmetric responses to returns is able to explain choice behavior of participants facing time series with either substantial or almost no structure (such as in the SI2, SI3 and WI2 blocks). For time series with a more complicated autocorrelation structure or a cyclical pattern (such as in the BH2 blocks) discrete choice models with multiple lags and/or asymmetric responses are required to give a good description of participants' decisions.

- The value of the IoC parameter increases with predictability of the time series. The estimated intensities of choice differ across time series. From a comparison of the estimated IoC parameters for data from the SI2 and SI3 blocks, with those obtained for the data from the WN2 blocks, it follows that these estimated coefficients are substantially higher when predictability in returns is higher.

- Standard discrete choice models fail to give a good description of participants' behavior when there are more than three alternatives. The experimental data from the SI4 and BH4 blocks cannot be satisfactorily described by a discrete choice model of the type (1) or $(2)$. 


\begin{tabular}{|c|c|c|c|c|c|c|c|}
\hline \multirow[b]{2}{*}{ 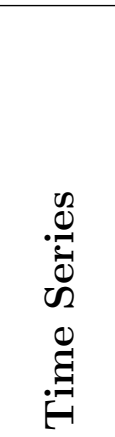 } & \multicolumn{2}{|c|}{ Best model from: } & \multicolumn{5}{|c|}{ Determinants of choice behavior } \\
\hline & 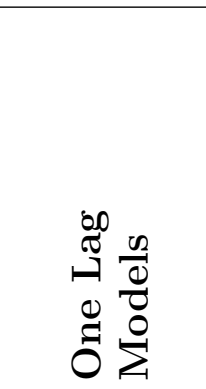 & 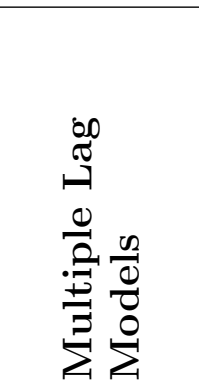 & 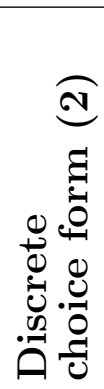 & 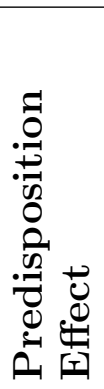 & 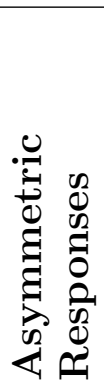 & 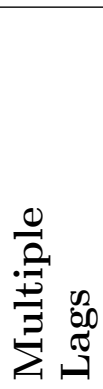 & 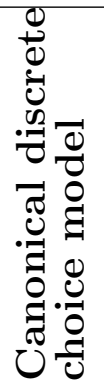 \\
\hline WN2 & PreSym1 & PreSym $3^{\dagger}$ & $\boldsymbol{V}$ & $\checkmark$ & $x$ & $x$ & $x$ \\
\hline $\mathrm{BH} 2$ & PreAsym1 & PreAsym 4 & $\checkmark$ & $\checkmark$ & $\checkmark$ & $\checkmark$ & $x$ \\
\hline ВH3 & Sym1 & - & $\checkmark$ & $x$ & $x$ & - & $\boldsymbol{V}$ \\
\hline $\mathrm{BH} 4$ & General1 & - & $x$ & $\checkmark$ & $\checkmark$ & - & $x$ \\
\hline SI2 & PreSym1 & PreSym1 & $\mathcal{V}$ & $\mathcal{V}$ & $x$ & $x$ & $x$ \\
\hline SI3 & PreSym1 & - & $\checkmark$ & $\mathcal{V}$ & $x$ & - & $x$ \\
\hline SI4 & General1 & - & $x$ & $\checkmark$ & $\checkmark$ & - & $x$ \\
\hline
\end{tabular}

† On the basis of the BIC, penalizing for extra parameters, we conclude that the PreSym3 model is not better than the PreSym1 model. Hence, WN2 does not require multiple lags.

Table 4: The most parsimonious model describing the data for every set of time series and an overview of the characteristics of the selected modelss.

Our interest in the discrete choice approach originates from its validity and viability as a building block of heterogeneous agent models, where it is used as a description of aggregate decisions. Therefore this paper studies whether the discrete choice model can explain the fraction of participants choosing a particular alternative. Alternatively, one could estimate a version of discrete choice model (1) or (2) on individual choices of participants. In that case the dependent variable is the probability that a particular individual chooses a certain alternative. This would be an interesting extension of our analysis. ${ }^{40}$ It could be used to see whether the discrete choice model gives a good description of every individual behavior and to characterize the heterogeneity of the estimated intensity of choice parameters across participants.

By considering exogenously generated time series of returns in the experiment we abstract from any influence the decisions of participants may have on the actual outcomes. However, aggregate market dynamics may be influenced by traders beliefs: if many investors select a particular stock, demand for that stock goes up and so will its price and return. One could design a laboratory experiment in which this feedback is incorporated. Payoffs of the participants will then also depend upon the decisions the other participants in the experiment make and it will be interesting to see whether, and how, this changes choice behavior.

\footnotetext{
${ }^{40}$ We only have 40 data points per participant, which is often not sufficient to get significant coefficients for the discrete choice model estimated on these data. There are also a few 'outliers', where, e.g., a participant does not react to the past returns by choosing the same fund every period, or where a participant always chooses the best fund, making the discrete choice model for this participant unidentifiable. Typically, therefore, the discrete choice models estimated on individual data are much more difficult to interpret than the model estimated on the aggregate data, where individual idiosyncrasies are smoothed out.
} 


\section{References}

Alevy J., Haigh M., List J., 2007. Information cascades: Evidence from a field experiment with financial market professionals. Journal of Finance 62, 151-180.

Alfarano S., Lux T., Wagner F., 2008. Time variation of higher moments in a financial market with heterogeneous agents: An analytical approach. Journal of Economic Dynamics \& Control 32, 101-136.

Anderson L., Holt C., 1997. Information cascades in the laboratory. American Economic Review 87, 847-862.

Anufriev M., Bao T., Sutan A., Tuinstra J., 2015. Fee structure, return chasing and mutual fund choice: An experiment. Available at SSRN: http://ssrn. com/abstract=2027157.

Anufriev M., Dindo P., 2010. Wealth-driven selection in a financial market with heterogeneous agents. Journal of Economic Behavior \& Organization 73, 327-358.

Anufriev M., Hommes C., 2012a. Evolution of market heuristics. Knowledge Engineering Review 27, $255-271$.

Anufriev M., Hommes C., 2012b. Evolutionary selection of individual expectations and aggregate outcomes in asset pricing experiments. American Economic Journal: Microeconomics $4,35-64$.

Anufriev M., Tuinstra J., 2013. The impact of short-selling constraints on financial market stability in a heterogeneous agents model. Journal of Economic Dynamics \& Control 37, $1523-1543$.

Bao T., Hommes C., Makarewicz T., 2016. Bubble formation and (in)efficient markets in learning-to-forecast and -optimise experiments. Economic Journal Forthcoming.

Bao T., Hommes C., Sonnemans J., Tuinstra J., 2012. Individual expectations, limited rationality and aggregate outcomes. Journal of Economic Dynamics \& Control 36, 1101-1120.

Boswijk P., Hommes C., Manzan S., 2007. Behavioral heterogeneity in stock prices. Journal of Economic Dynamics \& Control 31, 1938-1970.

Branch W., 2004. The theory of rationally heterogeneous expectations: Evidence from survey data on inflation expectations. Economic Journal 114, 592-621.

Brock W., Hommes C., 1997. A rational route to randomness. Econometrica 65, 1059-1095.

Brock W., Hommes C., 1998. Heterogeneous beliefs and routes to chaos in a simple asset pricing model. Journal of Economic Dynamics \& Control 22, 1235-1274.

Brock W.A., Hommes C.H., Wagener F.O.O., 2005. Evolutionary dynamics in markets with many trader types. Journal of Mathematical Economics 41, 7-42. 
Chiarella C., 1992. The dynamics of speculative behaviour. Annals of Operational Research $37,101-123$.

Chiarella C., Dieci R., He X.Z., 2007. Heterogeneous expectations and speculative behavior in a dynamic multi-asset framework. Journal of Economic Behavior \& Organization 62, 408-427.

Chiarella C., He X.Z., Huang W., Zheng H., 2012. Estimating behavioural heterogeneity under regime switching. Journal of Economic Behavior \& Organization 83, 446-460.

Chiarella C., He X.Z., Zwinkels R.C., 2014. Heterogeneous expectations in asset pricing: Empirical evidence from the S\&P500. Journal of Economic Behavior \& Organization 105, 1-16.

Choi J., Laibson D., Madrian B., Metrick A., 2009. Reinforcement learning and savings behavior. Journal of Finance 64, 2515-2534.

Day R., Huang W., 1990. Bulls, bears and market sheep. Journal of Economic Behaviour \& Organization 14, 299-329.

De Jong E., Verschoor W., Zwinkels R., 2009. Behavioural heterogeneity and shift-contagion: Evidence from the Asian crisis. Journal of Economic Dynamics \& Control 33, 1929-1944.

Drehmann M., Oechssler J., Roider A., 2005. Herding and contrarian behavior in financial markets: An internet experiment. American Economic Review 95, 1403-1426.

Fagiolo G., Roventini A., 2016. Macroeconomic policy in DSGE and agent-based models redux: New developments and challenges ahead. Technical report, Sant'Anna School of Advanced Studies. LEM Working Papers 2016/17.

Ferreira M.A., Keswani A., Miguel A.F., Ramos S.B., 2012. The flow-performance relationship around the world. Journal of Banking \& Finance 36, 1759-1780.

Franke R., Westerhoff F., 2012. Structural stochastic volatility in asset pricing dynamics: Estimation and model contest. Journal of Economic Dynamics \& Control 36, 1193-1211.

Gaunersdorfer A., Hommes C.H., Wagener F.O.O., 2008. Bifurcation routes to volatility clustering under evolutionary learning. Journal of Economic Behavior \& Organization 67, 27-47.

Goldbaum D., Mizrach B., 2008. Estimating the intensity of choice in a dynamic mutual fund allocation decision. Journal of Economic Dynamics \& Control 32, 3866-3876.

Goldbaum D., Panchenko V., 2010. Learning and adaptation's impact on market efficiency. Journal of Economic Behavior \& Organization 76, 635-653.

Heemeijer P., Hommes C., Sonnemans J., Tuinstra J., 2009. Price stability and volatility in markets with positive and negative expectations feedback: An experimental investigation. Journal of Economic Dynamics \& Control 33, 1052-1072. 
Hommes C., 2006. Heterogeneous agent models in economics and finance. In: K. Judd, L. Tesfatsion (Eds.), Handbook of Computational Economics Vol. 2: Agent-Based Computational Economics. Elsevier/North-Holland (Handbooks in Economics Series).

Hommes C., 2011. The heterogeneous expectations hypothesis: Some evidence from the lab. Journal of Economic Dynamics \& Control 35, 1-24.

Hommes C., 2013. Behavioral Rationality and Heterogeneous Expectations in Complex Economic Systems. Cambridge University Press.

Hommes C., Kiseleva T., Kuznetsov Y., Verbic M., 2012. Is more memory in evolutionary selection (de) stabilizing? Macroeconomic Dynamics 16, 335-357.

Hommes C., Sonnemans J., Tuinstra J., van de Velden H., 2005. Coordination of expectations in asset pricing experiments. Review of Financial Studies 18, 955-980.

Jain P., Wu J., 2000. Truth in mutual fund advertising: Evidence on future performance and fund flows. Journal of Finance 55, 937-958.

LeBaron B., 2006. Agent-based computational finance. In: K. Judd, L. Tesfatsion (Eds.), Handbook of Computational Economics Vol. 2: Agent-Based Computational Economics. Elsevier/North-Holland (Handbooks in Economics Series).

Lux T., 1995. Herd behaviour, bubbles and crashes. The Economic Journal 105, 881-896.

Lux T., 2009. Stochastic behavioral asset pricing models and the stylized facts. In: T. Hens, K.R. Schenk-Hoppé (Eds.), Handbook of Financial Markets: Dynamics and Evolution. NorthHolland.

Manski C., McFadden D., 1981. Alternative estimators and sample designs for discrete choice analysis. In: C. Manski, D. McFadden (Eds.), Structural Analysis of Discrete Data. MIT Press.

Manzan S., Westerhoff F.H., 2007. Heterogeneous expectations, exchange rate dynamics and predictability. Journal of Economic Behavior \& Organization 64, 111-128.

Pfajfar D., Žakelj B., 2014. Experimental evidence on inflation expectation formation. Journal of Economic Dynamics \& Control 44, 147-168.

Sirri E., Tufano P., 1998. Costly search and mutual fund flows. Journal of Finance 53, 15891622.

Zheng L., 1999. Is money smart? A study of mutual fund investors' fund selection ability. Journal of Finance 54, 901-933. 


\section{APPENDIX}

\section{A Experimental instructions}

In this experiment you will observe the time series of the financial returns of 2, 3 or 4 investment funds: fund A, B (or fund A, B, C, or fund A, B, C, D) for 100 consecutive periods. You will have to make an investment decision by choosing one of these funds. Your payoff in the experiment will depend on the return of the fund chosen as your investment decision.

The actual returns of the funds will always be positive. The information you have when making your forecasts and investment decision consists of the actual returns of the funds in the recent past. After each 50 periods of predictions, we will show you the returns of new funds, which might not have the same pattern as the former ones.

\section{Earnings}

You may earn points for every period of the experiment. The earned points will be transformed to the payoff in Euros at the end of the experiment. The number of points you get is the return from the fund you have chosen this period.

At the end of the experiment you are paid 1 euro for each 20 points you earned during the experiment. In addition you will get a show-up fee of 5 euros. As an example, if for one period you choose between 3 funds, and your choice and the actual returns are as in the table below: Then: you will get 3 points for this period, which is the actual return on fund A.

\begin{tabular}{c|c|c|c|c}
\hline The Fund You choose & Profit of Fund A & Profit of Fund B & Profit of Fund C & Your Payoff \\
\hline A & 3 & 1.3 & 4.6 & 3 \\
\hline
\end{tabular}




\section{B Details of the generated time series}

The data generating processes for time series WN2, SI2, SI3 and SI4 are described in the main text. Below we give the details for the time series $\mathrm{BH} 2, \mathrm{BH} 3$ and $\mathrm{BH} 4$. As explained in the main text, to meaningfully compare the Intensity of Choice parameter across treatments with different time series, we have to ensure that the values of returns are similar. For this purpose we normalize the time series from the $\mathrm{BH}$ and SI sets. The descriptive statistics of all time series (grouped by sets) is provided in Table 5 .

BH2 time series. To generate the returns we simulate a version of the Brock and Hommes (1997) model. Specifically, we used the Heuristic Switching Model from Anufriev and Hommes (2012b) with intensity of choice parameter $\beta=3.9$ and other parameters equal to $\eta=0.5$ and $\delta=0.2$. The demand, supply and cost parameters (see Brock and Hommes, 1997, p. 1066) are given by $A=0, B=0.5, b=1.35$ and $C=1$. To normalize we multiply both time series of returns by 4 and subsequently add 7 . The resulting set of time series is shown in Fig. 3 (upper left panel).

\begin{tabular}{|c|c|c|c|c|c|}
\hline Time series & Fund & Min & Mean & Max & Variance \\
\hline \multirow{3}{*}{ WN2 } & A & 2.17 & 5.68 & 9.37 & 3.13 \\
\hline & B & 0.60 & 5.16 & 10.25 & 4.48 \\
\hline & All & 0.60 & 5.42 & 10.25 & 3.83 \\
\hline \multirow{3}{*}{$\mathrm{BH} 2$} & $\mathrm{~A}$ & 4.50 & 5.24 & 9.42 & 1.38 \\
\hline & B & 0.47 & 6.01 & 7.75 & 5.42 \\
\hline & All & 0.47 & 5.63 & 9.42 & 3.52 \\
\hline \multirow{4}{*}{ BH3 } & $\mathrm{A}$ & 2.43 & 4.85 & 10.44 & 5.59 \\
\hline & B & 0.76 & 4.64 & 14.66 & 12.34 \\
\hline & $\mathrm{C}$ & 0.39 & 5.06 & 15.32 & 13.29 \\
\hline & All & 0.39 & 4.85 & 15.32 & 10.29 \\
\hline \multirow{5}{*}{$\mathrm{BH} 4$} & $\mathrm{~A}$ & 4.20 & 5.93 & 10.64 & 1.52 \\
\hline & B & 0.84 & 5.35 & 10.39 & 3.05 \\
\hline & $\mathrm{C}$ & 1.17 & 5.56 & 10.66 & 2.62 \\
\hline & $\mathrm{D}$ & 2.24 & 5.36 & 8.42 & 1.72 \\
\hline & All & 0.84 & 5.55 & 10.66 & 2.25 \\
\hline \multirow{3}{*}{ SI2 } & $\mathrm{A}$ & 0.36 & 5.62 & 10.72 & 9.42 \\
\hline & B & 0.93 & 5.18 & 7.89 & 5.73 \\
\hline & All & 0.36 & 5.40 & 10.72 & 7.55 \\
\hline \multirow{4}{*}{ SI3 } & $\mathrm{A}$ & 0.36 & 5.62 & 10.72 & 9.42 \\
\hline & B & 0.93 & 5.18 & 7.89 & 5.73 \\
\hline & $\mathrm{C}$ & 1.96 & 5.31 & 7.34 & 2.44 \\
\hline & All & 0.36 & 5.37 & 10.72 & 5.82 \\
\hline \multirow{5}{*}{ SI4 } & $\mathrm{A}$ & 0.36 & 5.62 & 10.72 & 9.42 \\
\hline & B & 0.93 & 5.18 & 7.89 & 5.73 \\
\hline & $\mathrm{C}$ & 1.96 & 5.31 & 7.34 & 2.44 \\
\hline & $\mathrm{D}$ & 2.59 & 5.44 & 7.62 & 2.23 \\
\hline & All & 0.36 & 5.39 & 10.72 & 4.91 \\
\hline
\end{tabular}

Table 5: Descriptive statistics of the returns time series used in the experiment. 
Funds $\mathrm{A}$ and $\mathrm{B}$ shown in the experiment have the following interpretation in terms of the original model. Fund A is run by a sophisticated manager that has rational expectations. This manager, however, charges a fee. Fund B is run by a manager using the naive expectations heuristic and charges no fee. The participant of our experiment does not know the strategies of the managers (and also does not know that there is a difference in sophistication between the fund managers), but only observes past returns (which also include the fee).

For the chosen parameters the model generates a repeating pattern of price fluctuations around the equilibrium, with a constantly growing amplitude, followed by a strong price correction. When the fluctuations are small, the naive forecasting heuristic is more profitable than the precise but expensive rational expectations heuristic. The agents in the model gradually switch from rational to the naive heuristic, which destabilizes the cobweb dynamics up to a point that fluctuations become so pronounced that the naive heuristic clearly performs worse than the rational heuristic. This makes agents switch back to the latter, which stabilizes the cobweb dynamics again, and so on. In the experiment we do not show the prices and forecasts, nor do we use non-neutral names for the different funds. Instead, we only show implied returns of the two heuristics (as in the left panel of Fig. 3) - framed as funds - in order to induce our participants to be motivated by the same forces as the agents in the original theoretical Brock and Hommes (1997) model.

BH3 and BH4 time series. The BH3 and BH4 time series are based on Brock and Hommes (1998). The three time series in BH3 are generated by the model with three forecasting heuristics, where one of the heuristics corresponds to fundamentalist behavior, and the two others represent biased predictors (optimists and pessimists, respectively, see Brock and Hommes, 1998, p. 1258). The time series were generated by simulating the model with the intensity of choice parameter $\beta=450$ and the other parameters given by $g_{1}=g_{2}=g_{3}=0, b_{1}=0$, $b_{2}=-b_{3}=0.1$ and $R=1.1$. This gives rise to quasi-cyclical dynamics. In order to inhibit predictability slightly we added an IID shock drawn from $N(0,0.01)$. Finally, we normalized the resulting returns by multiplying by 75 and adding 3 . The three resulting returns, $\pi_{A, t}, \pi_{B, t}$, and $\pi_{C, t}$ are shown in Fig. 3 (middle left panel).

The returns of fund A are those generated by the fundamental forecast, whose performance is always in between the performances of the two other funds. Funds B and $\mathrm{C}$ are symmetric with respect to $\mathrm{A}$, as their (opposite) biases are the same in absolute value. From time to time a different fund performs best.

The four time series in BH4 are also generated by the asset pricing model from Brock and Hommes (1998) but with four heuristics, representing fundamentalists, trend chasers with an upward bias, trend chasers with a downward bias and strong trend chasers with no bias, respectively (see Brock and Hommes, 1998, pp. 1261 - 63). The time series were generated by simulating the model with the intensity of choice parameter $\beta=95$ and the other parameters given by $b_{1}=b_{4}=0, b_{2}=-b_{3}=-0.3, g_{1}=0, g_{2}=1.1, g_{3}=0.9, g_{4}=1.21, C=0$ and $R=1.1$. We added small noise drawn from $N(0,0.0144)$. Finally, we normalized payoffs by multiplying them by 60 and adding 5.5. The resulting time series are shown in the left bottom panel of Fig. 3. 


\section{Estimation Results}

In this Section we collect the results of the estimation of various discrete choice models.

\section{C.1 Estimations for WN2 and BH2 time series}

Here we present the results of the estimation of the multiple lag model (5) from Section 5.2. Recall that in the first step (whose results are not shown), we choose the smallest possible lag $L$ after which an extra lag would lead to the model which is not significantly different from the current one (in the sense that the LR test would not reject the restriction $\beta_{A, L+1}=\beta_{B, L+1}=0$ ). In this step, we stop at $L=3$ for the WN2 blocks, at $L=4$ for the BH2 blocks and at $L=1$ for the SI2 blocks.

In the second step we estimate three different nested models with the selected lag $L$. We estimate (i) the unrestricted model (5), which is called PreAsymL, (ii) model (5) with restrictions $\beta_{A, \ell}=\beta_{B, \ell}$ for $\ell \leq L$, which is called PreSymL, and (iii) model (5) with restrictions $\beta_{A, \ell}=\beta_{B, \ell}$ for $\ell \leq L$ and $\alpha=0$, which is called SymL.

The results of this second step of the estimation procedure for the WN2 and BH2 blocks are presented in Tables 6 and 7, respectively. These tables have the same structure as Table 3 .

\begin{tabular}{c|cccc|ccccc}
\hline \hline Model & $\alpha$ & $\beta_{A, 1}$ & $\beta_{A, 2}$ & $\beta_{A, 3}$ & \multirow{2}{*}{ LL } & AIC & \multirow{2}{*}{ BIC } & $\begin{array}{c}\text { McF } \\
\text { R-sq }\end{array}$ & \multirow{2}{*}{ LR test } \\
and Lag & & $\beta_{B, 1}$ & $\beta_{B, 2}$ & $\beta_{B, 3}$ & & & & \\
\hline \hline \multirow{2}{*}{ Sym3 } & & 0.081 & -0.098 & -0.063 & -390.527 & 787.053 & 800.142 & -0.045 & \\
& & $(0.033)$ & $(0.033)$ & $(0.035)$ & & & & & \\
\hline \multirow{2}{*}{ PreSym3 } & 0.704 & 0.114 & -0.109 & -0.059 & -358.741 & 725.482 & $\mathbf{7 4 2 . 9 3 4}$ & 0.040 & 63.572 \\
& $(0.092)$ & $(0.035)$ & $(0.035)$ & $(0.037)$ & & & & & $(0.000)$ \\
\hline & 2.106 & 0.134 & -0.208 & -0.140 & -355.299 & $\mathbf{7 2 4 . 5 9 9}$ & 755.140 & 0.049 & 6.883 \\
PreAsym3 & $(0.879)$ & $(0.073)$ & $(0.060)$ & $(0.058)$ & & & & & $(0.332)$ \\
& & 0.115 & -0.103 & 0.018 & & & & & \\
& & $(0.039)$ & $(0.045)$ & $(0.051)$ & & & & & \\
\hline \hline
\end{tabular}

Table 6: Estimation and test results for the multi-lag models for the WN2 blocks. The last 20 periods of data are used (pooled over experience). Before generating this table, the PreAsym3 model was chosen as the most parsimonious asymmetric model with multiple lags, see the main text.

\begin{tabular}{|c|c|c|c|c|c|c|c|c|c|c|}
\hline $\begin{array}{c}\text { Model } \\
\text { and Lag }\end{array}$ & $\alpha$ & $\begin{array}{l}\beta_{A, 1} \\
\beta_{B, 1} \\
\end{array}$ & $\begin{array}{l}\beta_{A, 2} \\
\beta_{B, 2} \\
\end{array}$ & $\begin{array}{l}\beta_{A, 3} \\
\beta_{B, 3} \\
\end{array}$ & $\begin{array}{l}\beta_{A, 4} \\
\beta_{B, 4} \\
\end{array}$ & LL & AIC & BIC & $\begin{array}{l}\text { McF } \\
\text { R-sq }\end{array}$ & LR test \\
\hline Sym4 & & $\begin{array}{c}1.207 \\
(0.106)\end{array}$ & $\begin{array}{c}-0.738 \\
(0.076)\end{array}$ & $\begin{array}{c}0.239 \\
(0.103)\end{array}$ & $\begin{array}{l}-0.312 \\
(0.083)\end{array}$ & -209.796 & 427.592 & 445.044 & 0.420 & \\
\hline PreSym4 & $\begin{array}{l}-0.250 \\
(0.313)\end{array}$ & $\begin{array}{c}1.122 \\
(0.146)\end{array}$ & $\begin{array}{l}-0.673 \\
(0.108)\end{array}$ & $\begin{array}{c}0.173 \\
(0.138)\end{array}$ & $\begin{array}{l}-0.310 \\
(0.087)\end{array}$ & -209.463 & 428.925 & 450.740 & 0.421 & $\begin{array}{c}0.667 \\
(0.414)\end{array}$ \\
\hline PreAsym4 & $\begin{array}{c}9.223 \\
(64.671)\end{array}$ & $\begin{array}{l}10.896 \\
(2.032) \\
-8.931 \\
(1.886)\end{array}$ & $\begin{array}{c}0.717 \\
(1.677) \\
12.341 \\
(2.970)\end{array}$ & $\begin{array}{c}-16.112 \\
(4.976) \\
-2.422 \\
(3.699)\end{array}$ & $\begin{array}{c}3.366 \\
(3.366) \\
-0.259 \\
(-0.259)\end{array}$ & -189.981 & 397.962 & 437.229 & 0.475 & $\begin{array}{l}38.963 \\
(0.000)\end{array}$ \\
\hline
\end{tabular}

Table 7: Estimation and test results for multi-lag models for $\mathrm{BH} 2$ blocks. The last 20 periods of data are used. Before generating this table, the PreAsym4 model was chosen as the most parsimonious asymmetric models with multiple lags, see the main text. 


\section{C.2 Estimations for $\mathrm{BH} 3, \mathrm{BH} 4, \mathrm{SI} 3$ and SI4 time series}

In this section we present the results of the estimation of the one lag model with more than two alternatives from Section 6.

\begin{tabular}{|c|c|c|c|c|c|c|c|c|c|}
\hline Model & $\begin{array}{l}\alpha^{A}-\alpha^{C} \\
\alpha^{B}-\alpha^{C}\end{array}$ & $\begin{array}{c}\beta_{A}^{A}-\beta_{A}^{C} \\
\beta_{A}^{B}-\beta_{A}^{C}\end{array}$ & $\begin{array}{l}\beta_{B}^{A}-\beta_{B}^{C} \\
\beta_{B}^{B}-\beta_{B}^{C}\end{array}$ & $\begin{array}{l}\beta_{C}^{A}-\beta_{C}^{C} \\
\beta_{C}^{B}-\beta_{C}^{C}\end{array}$ & LL & AIC & BIC & $\begin{array}{l}\text { McF } \\
\text { R-sq }\end{array}$ & LR test \\
\hline Sym1 & -0.000 & $\begin{array}{c}2.008 \\
(0.158)\end{array}$ & 0.000 & $\begin{array}{c}-2.008 \\
\end{array}$ & -155.882 & 313.764 & 317.399 & 0.198 & \\
\hline PreSym1 & $\begin{array}{c}0.688 \\
(0.280) \\
-1.014 \\
(0.417)\end{array}$ & $\begin{array}{c}1.974 \\
(0.258) \\
0.000\end{array}$ & $\begin{array}{l}0.000 \\
1.974\end{array}$ & $\begin{array}{l}-1.974 \\
-1.974\end{array}$ & -139.343 & 284.687 & 295.591 & 0.283 & $\begin{array}{l}33.078 \\
(0.000)\end{array}$ \\
\hline PreAsym1 & $\begin{array}{l}-1.664 \\
(1.127) \\
-2.148 \\
(1.027)\end{array}$ & $\begin{array}{c}1.612 \\
(0.297) \\
0.000\end{array}$ & $\begin{array}{c}0.000 \\
1.261 \\
(0.431)\end{array}$ & $\begin{array}{l}-1.123 \\
(0.454) \\
-1.123\end{array}$ & -136.932 & 283.864 & 302.038 & 0.296 & $\begin{array}{c}4.823 \\
(0.090)\end{array}$ \\
\hline General1 & $\begin{array}{c}-0.890 \\
(1.280) \\
-2.604 \\
(2.096)\end{array}$ & $\begin{array}{c}1.501 \\
(0.377) \\
0.257 \\
(0.693)\end{array}$ & $\begin{array}{c}0.670 \\
(0.468) \\
0.818 \\
(0.863)\end{array}$ & $\begin{array}{c}-1.720 \\
(0.610) \\
-0.837 \\
(1.035)\end{array}$ & -135.258 & 286.517 & 315.595 & 0.304 & $\begin{array}{c}3.348 \\
(0.341)\end{array}$ \\
\hline
\end{tabular}

Table 8: Estimation of multivariate logit models for the experimental data from the SI3 block. The last 20 periods are used. The last column shows the results of LR test of the corresponding model against the previous (restricted) model.

\begin{tabular}{|c|c|c|c|c|c|c|c|c|c|c|}
\hline Model & $\begin{array}{l}\alpha^{A}-\alpha^{D} \\
\alpha^{B}-\alpha^{D} \\
\alpha^{C}-\alpha^{D}\end{array}$ & $\begin{array}{c}\beta_{A}^{A}-\beta_{A}^{D} \\
\beta_{A}^{B}-\beta_{A}^{D} \\
\beta_{A}^{C}-\beta_{A}^{D}\end{array}$ & $\begin{array}{l}\beta_{B}^{A}-\beta_{B}^{D} \\
\beta_{B}^{B}-\beta_{B}^{D} \\
\beta_{B}^{C}-\beta_{B}^{D}\end{array}$ & $\begin{array}{l}\beta_{C}^{A}-\beta_{C}^{D} \\
\beta_{C}^{B}-\beta_{C}^{D} \\
\beta_{C}^{C}-\beta_{C}^{D}\end{array}$ & $\begin{array}{l}\beta_{D}^{A}-\beta_{D}^{D} \\
\beta_{D}^{D}-\beta_{D}^{D} \\
\beta_{D}^{C}-\beta_{D}^{D}\end{array}$ & LL & AIC & BIC & $\begin{array}{l}\text { McF } \\
\text { R-sq }\end{array}$ & LR test \\
\hline Sym1 & 0.000 & $\begin{array}{c}2.694 \\
(0.162)\end{array}$ & -0.000 & -0.000 & -2.694 & -418.554 & 839.109 & 843.437 & 0.259 & \\
\hline PreSym1 & $\begin{array}{c}1.303 \\
(0.234) \\
-0.602 \\
(0.582) \\
-0.078 \\
(0.113)\end{array}$ & $\begin{array}{c}3.072 \\
(0.248) \\
0.000 \\
\\
0.000\end{array}$ & $\begin{array}{l}0.000 \\
3.072 \\
0.000\end{array}$ & $\begin{array}{l}0.000 \\
0.000 \\
3.072\end{array}$ & $\begin{array}{l}-3.072 \\
-3.072 \\
-3.072\end{array}$ & -391.487 & 790.974 & 808.286 & 0.307 & $\begin{array}{l}54.135 \\
(0.000)\end{array}$ \\
\hline PreAsym1 & $\begin{array}{c}5.630 \\
(0.958) \\
0.660 \\
(1.510) \\
-0.453 \\
(0.503)\end{array}$ & $\begin{array}{c}3.186 \\
(0.234) \\
-0.000 \\
\\
0.000\end{array}$ & $\begin{array}{c}0.000 \\
3.915 \\
(0.390) \\
-0.000\end{array}$ & $\begin{array}{c}0.000 \\
-0.000 \\
4.204 \\
(0.341)\end{array}$ & $\begin{array}{l}-4.059 \\
(0.366) \\
-4.059 \\
-4.059\end{array}$ & -374.731 & 763.463 & 793.758 & 0.337 & $\begin{array}{l}33.511 \\
(0.000)\end{array}$ \\
\hline General1 & $\begin{array}{c}5.305 \\
(3.551) \\
24.027 \\
(13.552) \\
7.385 \\
(2.880)\end{array}$ & $\begin{array}{c}1.407 \\
(0.492) \\
1.573 \\
(2.415) \\
0.500 \\
(0.436)\end{array}$ & $\begin{array}{c}2.982 \\
(0.817) \\
10.230 \\
(4.974) \\
1.875 \\
(0.621)\end{array}$ & $\begin{array}{c}1.004 \\
(0.664) \\
-4.162 \\
(3.931) \\
5.497 \\
(0.720)\end{array}$ & $\begin{array}{c}-5.677 \\
(1.243) \\
-11.678 \\
(5.213) \\
-8.944 \\
(1.080)\end{array}$ & -337.499 & 704.999 & 769.918 & 0.403 & $\begin{array}{l}74.464 \\
(0.000)\end{array}$ \\
\hline
\end{tabular}

Table 9: Estimation of the multivariate logit models for the data from the SI4 blocks. The last 20 periods are used (pooled over experienced and inexperienced participants). The last column shows the results of LR test of the corresponding model against the previous (restricted) model.

Tables 8 and 9 give the results for the SI3 and SI4 blocks, respectively. The estimated coefficients are presented in matrix form, with a description of the relevant parameters given in the first row of these tables (this description is based upon the specification in Eq. (7)). The last row of the tables shows the estimated General1 models. For the restricted models (except for the Sym1 model, which has only one parameter) we also show the values of all parameters (with the value equal to 0 if the parameter is restricted to be 0 ), but we only report standard errors once across parameters that are restricted to be the same. As before, the last column of the tables should be read from bottom to top. It shows the LR statistics and $p$-value for the hypothesis consisting of the restrictions leading to the model in the row immediately above. 


\begin{tabular}{|c|c|c|c|c|c|c|c|c|}
\hline Model & $\begin{array}{l}\alpha^{A}-\alpha^{C} \\
\alpha^{B}-\alpha^{C}\end{array}$ & $\begin{array}{l}\tilde{\beta}_{B}^{A}-\tilde{\beta}_{B}^{C} \\
\tilde{\beta}_{B}^{B}-\tilde{\beta}_{B}^{C}\end{array}$ & $\begin{array}{l}\tilde{\beta}_{C}^{A}-\tilde{\beta}_{C}^{C} \\
\tilde{\beta}_{C}^{B}-\tilde{\beta}_{C}^{C}\end{array}$ & LL & AIC & BIC & $\begin{array}{l}\text { McF } \\
\text { R-sq }\end{array}$ & LR test \\
\hline Sym1 & 0.000 & 0.000 & $\begin{array}{l}-0.039 \\
(0.012)\end{array}$ & -654.266 & 1310.531 & 1314.928 & 0.005 & \\
\hline PreSym1 & $\begin{array}{c}0.110 \\
(0.135) \\
0.063 \\
(0.099)\end{array}$ & $\begin{array}{l}0.000 \\
0.047\end{array}$ & $\begin{array}{l}-0.047 \\
(0.017) \\
-0.047\end{array}$ & -653.891 & 1313.781 & 1326.972 & 0.006 & $\begin{array}{c}0.750 \\
(0.687)\end{array}$ \\
\hline PreAsym1 & $\begin{array}{c}0.175 \\
(0.162) \\
0.192 \\
(0.199) \\
\end{array}$ & $\begin{array}{c}0.000 \\
\\
0.033 \\
(0.026) \\
\end{array}$ & $\begin{array}{l}-0.059 \\
(0.024) \\
-0.059\end{array}$ & -653.612 & 1315.224 & 1332.811 & 0.006 & $\begin{array}{c}0.558 \\
(0.455)\end{array}$ \\
\hline General1 & $\begin{array}{c}-0.230 \\
(0.235) \\
0.181 \\
(0.227)\end{array}$ & $\begin{array}{c}0.061 \\
(0.032) \\
0.060 \\
(0.031)\end{array}$ & $\begin{array}{c}-0.029 \\
(0.028) \\
-0.080 \\
(0.029)\end{array}$ & -650.573 & 1313.147 & 1339.528 & 0.011 & $\begin{array}{c}6.077 \\
(0.048)\end{array}$ \\
\hline
\end{tabular}

Table 10: Estimation of multivariate logit model for BH3. Last 20 periods of data are used (pooled over experiences). The last column shows the results of LR test of the corresponding model against the previous (restricted) model.

\begin{tabular}{|c|c|c|c|c|c|c|c|c|c|}
\hline Model & $\begin{array}{l}\alpha^{A}-\alpha^{D} \\
\alpha^{B}-\alpha^{D} \\
\alpha^{C}-\alpha^{D}\end{array}$ & $\begin{array}{c}\tilde{\beta}_{B}^{A}-\tilde{\beta}_{B}^{D} \\
\tilde{\beta}_{B}^{B}-\tilde{\beta}_{B}^{D} \\
\tilde{\beta}_{B}^{C}-\tilde{\beta}_{B}^{D}\end{array}$ & $\begin{array}{l}\tilde{\beta}_{C}^{A}-\tilde{\beta}_{C}^{D} \\
\tilde{\beta}_{C}^{B}-\tilde{\beta}_{C}^{D} \\
\tilde{\beta}_{C}^{C}-\tilde{\beta}_{C}^{D}\end{array}$ & $\begin{array}{l}\tilde{\beta}_{D}^{A}-\tilde{\beta}_{D}^{D} \\
\tilde{\beta}_{D}^{B}-\tilde{\beta}_{D}^{D} \\
\tilde{\beta}_{D}^{C}-\tilde{\beta}_{D}^{D}\end{array}$ & LL & AIC & BIC & $\begin{array}{l}\text { McF } \\
\text { R-sq }\end{array}$ & LR test \\
\hline Sym1 & 0.000 & 0.000 & $\overline{-0.000}$ & $\begin{array}{c}0.031 \\
(0.022) \\
\end{array}$ & -387.197 & 7776.395 & $\overline{7780.029}$ & $\overline{-0.241}$ & \\
\hline PreSym1 & $\begin{array}{c}5.590 \\
(0.762) \\
3.444 \\
(0.718) \\
3.806 \\
(0.715)\end{array}$ & $\begin{array}{c}0.000 \\
0.302 \\
-0.000\end{array}$ & $\begin{array}{c}0.000 \\
-0.000 \\
0.302\end{array}$ & $\begin{array}{l}-0.302 \\
(0.051) \\
-0.302 \\
-0.302\end{array}$ & -292.944 & 593.888 & 608.427 & 0.061 & $\begin{array}{l}188.507 \\
(0.000)\end{array}$ \\
\hline PreAsym1 & $\begin{array}{c}9.132 \\
(3.538) \\
7.247 \\
(3.552) \\
7.120 \\
(3.531)\end{array}$ & $\begin{array}{c}0.000 \\
\\
0.258 \\
(0.072) \\
0.000\end{array}$ & $\begin{array}{c}0.000 \\
0.000 \\
0.341 \\
(0.071)\end{array}$ & $\begin{array}{l}-0.863 \\
(0.500) \\
-0.863 \\
\\
-0.863\end{array}$ & -291.893 & 595.785 & 617.594 & 0.065 & $\begin{array}{c}2.103 \\
(0.349)\end{array}$ \\
\hline General1 & $\begin{array}{c}1.074 \\
(8.101) \\
2.445 \\
(8.115) \\
2.656 \\
(8.103)\end{array}$ & $\begin{array}{c}11.338 \\
(12.651) \\
8.423 \\
(12.656) \\
8.539 \\
(12.653)\end{array}$ & $\begin{array}{c}11.327 \\
(13.358) \\
7.771 \\
(13.366) \\
9.194 \\
(13.359)\end{array}$ & $\begin{array}{l}-22.185 \\
(24.731) \\
-15.924 \\
(24.743) \\
-17.498 \\
(24.732)\end{array}$ & -256.207 & 536.415 & 580.032 & 0.179 & $\begin{array}{l}71.371 \\
(0.000)\end{array}$ \\
\hline
\end{tabular}

Table 11: Estimation of multivariate logit model for BH4. Last 20 periods of data are used. The last column shows the results of LR test of the corresponding model against the previous (restricted) model.

Before presenting the results for BH3 and BH4 blocks, recall from Section 6.2 that in this case the General1 model has the special form (8). By imposing the restrictions $\tilde{\beta}_{k}^{h}=\tilde{\beta}_{k}^{l}$ for all $h, k$ and $l$ different from each other, model (8) reduces to the PreAsym1 model

$n_{t}^{A}=\frac{\exp \left[\alpha_{A}\right]}{Z}, n_{t}^{B}=\frac{\exp \left[\alpha_{B}+\tilde{\beta}_{B} \pi_{B, t-1}\right]}{Z}, Z=\exp \left[\alpha_{A}\right]+\exp \left[\alpha_{B}+\tilde{\beta}_{B} \pi_{B, t-1}\right]+\exp \left[\tilde{\beta}_{C} \pi_{C, t-1}\right]$,

where $\alpha_{A}=\alpha^{A}-\alpha^{C}, \alpha_{B}=\alpha^{B}-\alpha^{C}, \tilde{\beta}_{B}=\tilde{\beta}_{B}^{B}-\tilde{\beta}_{B}^{C}$ and $\tilde{\beta}_{C}=-\left(\tilde{\beta}_{C}^{B}-\tilde{\beta}_{C}^{C}\right)$. This PreAsym1 model does not include the intensity of choice for alternative A. Indeed, we cannot identify all of the intensities of choice simultaneously; and the way how we reduced parameters in (8) to obtain identification, dictates that the intensity of choice for $\mathrm{A}$ is absent. ${ }^{41}$ With the additional

\footnotetext{
${ }^{41}$ If we would express performance of B (resp. C) as a linear combination of the performances of $\mathrm{A}$ and $\mathrm{C}$ (resp. A and B), then the PreAsym1 model would not have the intensity of choice for B (resp. C).
} 
assumption $\tilde{\beta}=\tilde{\beta}_{B}=\tilde{\beta}_{C}$ we obtain the PreSym1 model given by

$$
n_{t}^{A}=\frac{\exp \left[\alpha_{A}\right]}{Z}, n_{t}^{B}=\frac{\exp \left[\alpha_{B}+\tilde{\beta} \pi_{B, t-1}\right]}{Z}, Z=\exp \left[\alpha_{A}\right]+\exp \left[\alpha_{B}+\tilde{\beta} \pi_{B, t-1}\right]+\exp \left[\tilde{\beta} \pi_{C, t-1}\right]
$$

Finally, the Sym1 model - where predisposition effects are absent - reads

$$
n_{t}^{A}=\frac{1}{Z}, \quad n_{t}^{B}=\frac{\exp \left[\tilde{\beta} \pi_{B, t-1}\right]}{Z}, \quad Z=1+\exp \left[\tilde{\beta} \pi_{B, t-1}\right]+\exp \left[\tilde{\beta} \pi_{C, t-1}\right] .
$$

Tables 10 and 11 give the results of estimation for the BH3 and BH4 blocks, respectively. 


\section{ON-LINE APPENDIX (not for publication)}

\section{Testing the effect of experience}

We analyze the effect of experience for a given time series, by comparing the aggregate behavior of participants who experience this time series in the first block of their treatment and those who experience this time series in the second block of their treatment. Given the design of treatments, we can do this for the WN2, BH2, BH3, SI2 and SI4 time series.

Note that there are two ways to look at the decisions that participants make over time in our experiment. On the one hand, using participants' choices between alternatives, we can construct the evolution of the share of participants choosing a particular alternative by aggregating over all participants in the block. On the other hand, we can focus on those instances when a participant has switched, i.e., when a participant's choice in a given period differed from his or her choice in the previous period, and construct the empirical distribution of the number of switches. The left (middle) column of Fig. 9 contains the panels with the area charts plotting the evolution of the shares of inexperienced (experienced) participants choosing a particular alternative. The right column contains the normalized histograms of number of switches for a particular set of time series (separately for inexperienced and experienced participants). To get a better idea of the frequency with which participants switch between alternatives we count the number of switches for each treatment. Because the number of participants varies with the treatments, we use the number of switches per participant per period as a measure to compare different blocks. The results are shown in Table 12. This normalized fraction of switches lies between 21\% (SI2, Experienced) and 55\% (BH3, Inexperienced).

A visual comparison between the left and middle panels of Fig. 9 suggests that the effect of experience is limited. Choice behavior of experienced participants seems to be quite similar,

\begin{tabular}{c|c|c|c}
\hline \hline Time-series & Block, Treatment & \multicolumn{2}{|c}{ Switches } \\
& & Total number & per participant per period \\
\hline \hline \multirow{2}{*}{ WN2 } & 1 (Inexperienced), T1 & 210 & $32.81 \%$ \\
& 2 (Experienced), T6 & 224 & $43.08 \%$ \\
\hline \multirow{2}{*}{ BH2 } & 1 (Inexperienced), T2 & 159 & $26.50 \%$ \\
& 2 (Experienced), T5 & 145 & $25.89 \%$ \\
\hline \multirow{2}{*}{ BH3 } & 1 (Inexperienced), T3 & 308 & $55.00 \%$ \\
& 2 (Experienced), T1 & 332 & $51.88 \%$ \\
\hline BH4 & 2 (Experienced), T4 & 203 & $36.25 \%$ \\
\hline \multirow{2}{*}{ SI2 } & 1 (Inexperienced), T4 & 147 & $26.25 \%$ \\
& 2 (Experienced), T3 & 119 & $21.25 \%$ \\
\hline SI3 & 1 (Inexperienced), T5 & 169 & $30.18 \%$ \\
\multirow{2}{*}{ SI4 } & 1 (Inexperienced), T6 & 218 & $41.92 \%$ \\
& 2 (Experienced), T2 & 251 & $41.83 \%$ \\
\hline \hline
\end{tabular}

Table 12: Number of switches for each block in the experiment. 

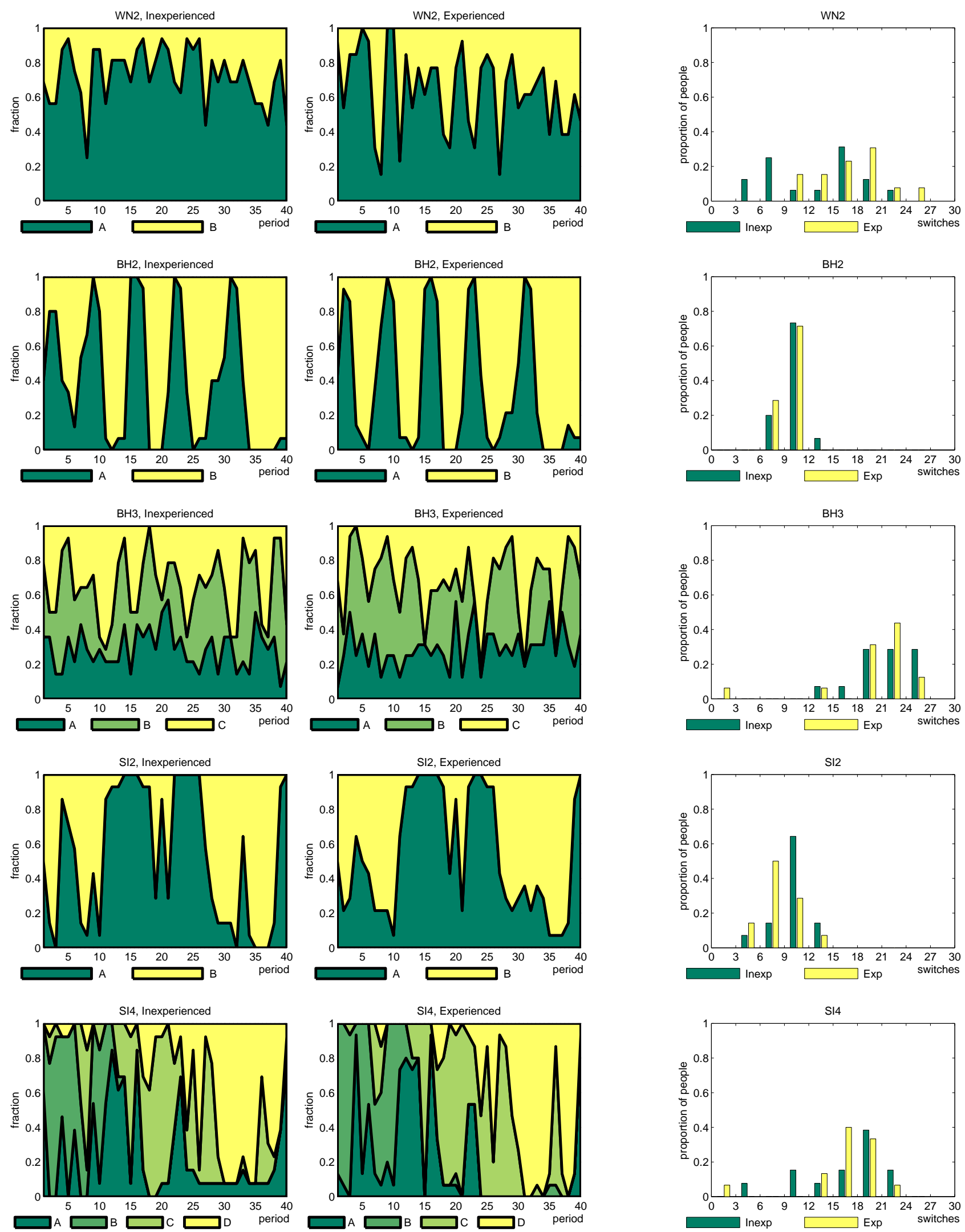

Figure 9: Shares of choices over time for inexperienced subjects (left panels) and for experienced subjects (middle panels), and distributions of number of switches in a session (right panels). From top to bottom: WN2, BH2, BH3, SI2 and SI4. 
for a given set of time series, to that of inexperienced participants.

To test Hypothesis 1 formally we introduce the following notation. For each time period $t=1, \ldots, 40$ and for every alternative $h$ (with $h$ being A, B, C or D) we observe the shares of choices $s_{t, h}^{\text {InExp }}$ and $s_{t, h}^{\text {Exp }}$ made by the inexperienced and experienced participants, respectively. Under the null hypothesis that the discrete choices in these two populations are drawn from an identical multinomial distribution we can compute the actual, $n$, and "expected", $\hat{n}$, number of choices of each of the alternatives in the inexperienced and experienced sessions:

$$
\begin{aligned}
n_{t, h}^{\mathrm{InExp}} & =s_{t, h}^{\mathrm{InExp}} \cdot N^{\mathrm{InExp}}, & \hat{n}_{t, h}^{\mathrm{InExp}} & =\left(n_{t, h}^{\operatorname{InExp}}+n_{t, h}^{\operatorname{Exp}}\right) \cdot \frac{N^{\mathrm{InExp}}}{N^{\operatorname{InExp}}+N^{\operatorname{Exp}}}, \\
n_{t, h}^{\operatorname{Exp}} & =s_{t, h}^{\operatorname{Exp}} \cdot N^{\operatorname{Exp}}, & \hat{n}_{t, h}^{\operatorname{Exp}} & =\left(n_{t, h}^{\operatorname{InExp}}+n_{t, h}^{\operatorname{Exp}}\right) \cdot \frac{N^{\operatorname{Exp}}}{N^{\operatorname{InExp}}+N^{\operatorname{Exp}}} .
\end{aligned}
$$

where $N^{\operatorname{InExp}}$ and $N^{\operatorname{Exp}}$ are the numbers of participants in the "inexperienced" and "experienced" blocks, respectively. Under the null hypothesis Pearson's statistic

$$
\sum_{h} \frac{\left(n_{t, h}^{\mathrm{InExp}}-\hat{n}_{t, h}^{\mathrm{InExp}}\right)^{2}}{\hat{n}_{t, h}^{\mathrm{InExp}}}+\sum_{h} \frac{\left(n_{t, h}^{\mathrm{Exp}}-\hat{n}_{t, h}^{\mathrm{Exp}}\right)^{2}}{\hat{n}_{t, h}^{\mathrm{Exp}}}
$$

is distributed according to the Chi-squared distribution with $H-1$, degrees of freedom, where

\begin{tabular}{|c|c|c|c|c|c|c|c|c|c|c|c|}
\hline Period & $\overline{\text { WN2 }}$ & $\overline{\mathrm{BH} 2}$ & BH3 & 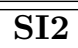 & SI4 & Period & $\overline{\text { WN2 }}$ & $\overline{\mathrm{BH} 2}$ & BH3 & SI2 & $\overline{\text { SI4 }}$ \\
\hline 1 & 0.88 & 0.12 & 0.00 & 0.87 & 0.93 & 21 & 0.33 & 0.53 & 0.34 & 0.96 & 0.25 \\
\hline 2 & 0.10 & 0.68 & 0.38 & 0.22 & 0.79 & 22 & 0.78 & 0.71 & 0.69 & 0.23 & 0.35 \\
\hline 3 & 0.90 & 0.32 & 0.97 & 0.98 & 0.00 & 23 & 0.91 & 0.67 & 0.00 & 0.93 & 0.14 \\
\hline 4 & 0.18 & 0.88 & 0.81 & 0.74 & 0.95 & 24 & 0.81 & 0.40 & 0.00 & 0.43 & 0.68 \\
\hline 5 & 0.64 & 0.92 & 0.75 & 0.40 & 0.59 & 25 & 0.18 & 0.71 & 0.69 & 0.52 & 0.54 \\
\hline 6 & 0.78 & 0.84 & 0.55 & 0.02 & 0.11 & 26 & 0.81 & 0.67 & 0.69 & 0.65 & 0.41 \\
\hline 7 & 0.91 & 0.66 & 0.38 & 0.39 & 0.43 & 27 & 0.90 & 0.04 & 0.55 & 0.29 & 0.25 \\
\hline 8 & 0.48 & 0.22 & 0.72 & 0.81 & 0.91 & 28 & 0.55 & 0.72 & 0.00 & 0.57 & 0.38 \\
\hline 9 & 0.81 & 0.00 & 0.78 & 0.74 & 0.68 & 29 & 0.68 & 0.72 & 0.38 & 0.37 & 0.72 \\
\hline 10 & 0.81 & 0.32 & 0.00 & 0.94 & 0.53 & 30 & 0.89 & 0.14 & 0.64 & 0.48 & 0.82 \\
\hline 11 & 0.93 & 0.04 & 0.81 & 0.86 & 0.24 & 31 & 0.32 & 0.00 & 0.81 & 0.42 & 0.25 \\
\hline 12 & 0.19 & 0.71 & 0.00 & 0.93 & 0.01 & 32 & 0.32 & 0.04 & 0.93 & 0.68 & 0.25 \\
\hline 13 & 0.89 & 0.67 & 0.00 & 0.19 & 0.08 & 33 & 0.55 & 0.72 & 0.87 & 0.55 & 0.53 \\
\hline 14 & 0.23 & 0.04 & 0.00 & 0.74 & 0.07 & 34 & 0.38 & 0.00 & 0.86 & 0.54 & 0.25 \\
\hline 15 & 0.32 & 0.71 & 0.00 & 0.97 & 0.52 & 35 & 0.66 & 0.00 & 0.69 & 0.48 & 0.59 \\
\hline 16 & 0.55 & 0.00 & 0.00 & 0.86 & 0.09 & 36 & 0.53 & 0.00 & 0.69 & 0.59 & 0.66 \\
\hline 17 & 0.81 & 0.50 & 0.69 & 0.38 & 0.25 & 37 & 0.23 & 0.00 & 0.69 & 0.66 & 0.38 \\
\hline 18 & 0.90 & 0.00 & 0.00 & 0.93 & 0.39 & 38 & 0.90 & 0.87 & 0.00 & 0.04 & 0.72 \\
\hline 19 & 0.99 & 0.00 & 0.57 & 0.46 & 0.43 & 39 & 0.76 & 0.04 & 0.46 & 0.46 & 0.50 \\
\hline 20 & 0.81 & 0.00 & 0.00 & 0.53 & 0.18 & 40 & 0.10 & 0.04 & 0.00 & 0.64 & 0.53 \\
\hline
\end{tabular}
$H$ is the number of alternatives. Table 13 shows the $p$-value of this statistic for different time periods and sets of time series. We reject the null hypothesis, at the $5 \%$ significance level, only for $t=19$ in WN2, for $t=3$ in SI2, and for $t=3, t=15$ and $t=21$ in BH3. We are never able to reject the null hypothesis in $\mathrm{BH} 2$ and SI4.

Table 13: $p$-values for Pearson chi-squared statistics. 


\section{E Efficiency and Learning}

Analysis of performance in Section 4.2 focuses on the number of the best choices made by participants. This measure is not perfect for the blocks with more than two alternatives, because participants could earn more with the second best alternative than with the third or fourth best alternative. To deal with this issue, we define the efficiency measure Eff as

$$
\mathbf{E f f}=\frac{\text { Average Experimental Earnings }- \text { Expected Earnings when Choosing Randomly }}{\text { Earnings when Choosing Optimally }- \text { Expected Earnings when Choosing Randomly }}
$$

If this measure is close to 1 it means that participants almost always make optimal decisions, whereas if it is close to 0 participants' performance is not much better than their expected payoff when they choose an alternative randomly (note that Eff can become negative if participants earn less points than they would earn, in expectation, when choosing randomly).

Table 14 presents this measure for the different sets of time series (the sixth column of the table). It also presents the maximum and minimum number of points that could be earned, the expected number of points under random choice and the actual average number of points earned. By construction, there is no structure or predictability in the time series from WN2, and indeed efficiency, although still positive, is the lowest there. The time series from BH3 show little structure as well and participants find it difficult to make correct choices for those time series. For the other five sets of time series the efficiency level is between $63 \%$ and $78 \%$.

The last two columns of Table 14 focus on possible learning by indirectly evaluating its consequences. These columns give, for each set of time series, the value of the efficiency measure Eff separately for the first 20 periods and the last 20 periods in the blocks. Note that for WN2 efficiency goes down in the last twenty periods and even becomes negative, but since the evolution of the WN2 returns series is, by construction, unpredictable we would not expect any learning to take place in these blocks anyway. In fact, it is surprising that, by chance, participants reach a quite reasonable level of efficiency in the first 20 periods. For five of the other six sets of time series there is a substantial increase in efficiency, with the measure increasing by 10 to 24 percentage points, which suggests that, during the block, participants learn to make better decisions. The only surprising result in this respect is that the efficiency

\begin{tabular}{c||cccc||c|cc}
\hline \hline \multirow{2}{*}{ Time series } & \multirow{2}{*}{ Max } & \multirow{2}{*}{ Min } & \multirow{2}{*}{ Random } & \multirow{2}{*}{ Experiment } & \multicolumn{3}{c}{ Efficiency } \\
& & & & & Total & First 20 & Last 20 \\
\hline \hline WN2 & 271.19 & 179.65 & 225.42 & 231.27 & $12.80 \%$ & $32.19 \%$ & $-5.33 \%$ \\
\hline BH2 & 285.30 & 161.08 & 223.19 & 269.87 & $75.16 \%$ & $66.87 \%$ & $82.36 \%$ \\
BH3 & 258.71 & 105.16 & 181.94 & 200.10 & $23.65 \%$ & $16.60 \%$ & $28.64 \%$ \\
BH4 & 263.59 & 179.88 & 223.22 & 249.50 & $65.09 \%$ & $71.31 \%$ & $59.20 \%$ \\
\hline SI2 & 197.22 & 175.81 & 186.51 & 193.32 & $63.55 \%$ & $58.11 \%$ & $68.08 \%$ \\
SI3 & 217.82 & 170.72 & 193.03 & 210.89 & $72.04 \%$ & $56.39 \%$ & $80.42 \%$ \\
SI4 & 228.81 & 166.25 & 195.49 & 216.38 & $76.46 \%$ & $70.76 \%$ & $80.15 \%$ \\
\hline \hline
\end{tabular}

Table 14: The average payoff, payoffs under different scenarios and efficiency for each type of time series. Data from experienced and inexperienced participants are pooled. 

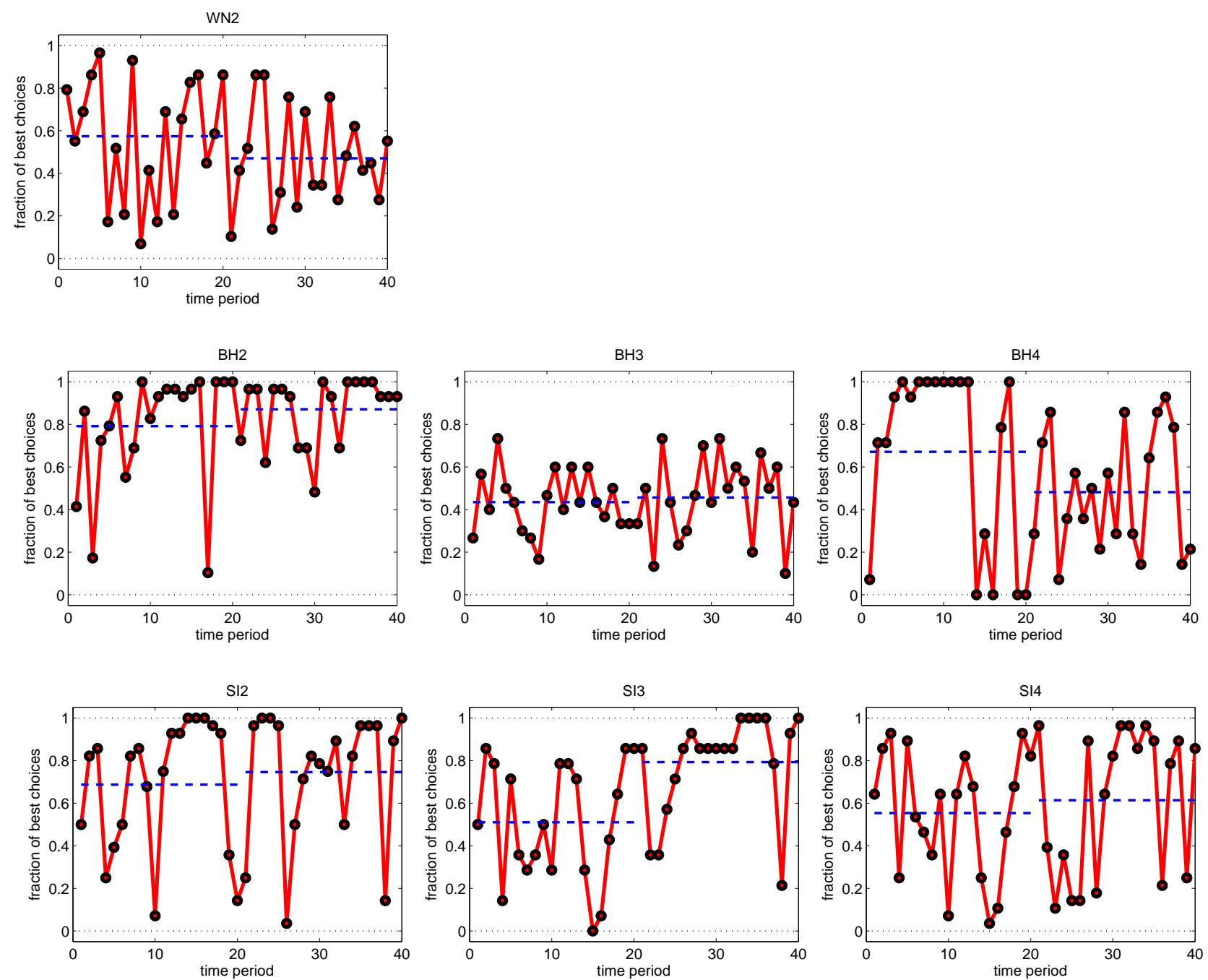

Figure 10: The fraction of participants choosing the best fund. Inexperienced and experienced sessions are pooled together. The horizontal dashed lines show the averages of the fractions over the first 20 and last 20 periods.

for the BH4 set of time series goes substantially down in the last 20 periods: apparently learning for that set of time series is particularly challenging.

In addition to the averages presented in Table 2, Figure 10 shows how the percentage of participants choosing the best fund was changing over time (the horizontal dashed lines correspond to the averages reported in Table 2). 


\section{F Estimation of Discrete Choice Models}

In Section 5 we have presented results on estimating the standard logit model on choices between two, three or four alternatives, with the past performance of these alternatives as explanatory variables. In this appendix we elaborate on the underlying models and estimation procedures.

The most general version of the multivariate logit model predicts that the probability of choosing alternative $h$ at time $t$ by participant $i$, denoted by $\operatorname{Pr}\left(c_{i, t}=h\right)$, is proportional to the exponent of a linear combination of past payoffs of all alternatives, i.e., all information available to the participant. We again use capital letters $A, B, C$ and $D$ for the different alternatives but abuse notation slightly by denoting the last alternative by $H$ (so that $H$ is $B, C$ or $D$ when there are two, three or four alternatives, respectively) and the penultimate alternative by $H^{-}$ (so that $H^{-}$denotes $A, B$ or $C$, respectively). The most general model then has

$$
\operatorname{Pr}\left(c_{i, t}=h\right) \propto \exp \left[\alpha^{h}+\beta_{A, 1}^{h} \pi_{A, t-1}+\cdots+\beta_{H, 1}^{h} \pi_{H, t-1}+\beta_{A, 2}^{h} \pi_{A, t-2}+\cdots+\beta_{H, 2}^{h} \pi_{H, t-2}+\ldots\right],
$$

for any alternative $h$. Here $\alpha^{h}$ and the $\beta_{k, \ell}^{h}$ 's are the coefficients to be estimated, where $\ell$ indicates the lag (which can go up to a fixed maximum lag $L$ ). Coefficient $\beta_{k, \ell}^{h}$ measures the sensitivity of choice of alternative $h$ with respect to alternative $k$ 's payoff $\ell$ periods ago. Note that the model is indeterminate since adding, for a given other alternative $k$ and lag $l$, the same constant to the sensitivities of choices for each of the alternatives (i.e., for given $k$ and $\ell$ change $\beta_{k, \ell}^{h}$ to $\widetilde{\beta}_{k, \ell}^{h}=\beta_{k, \ell}^{h}+\xi$ for $\left.h=A, B, \ldots, H\right)$ will not change the probabilities (the same reasoning holds for the $\alpha^{h}$ ).

We can therefore choose one alternative as the "reference option" (in our case alternative $H$ ) and rewrite expression (9) for all $h=A, B, \ldots, H$ as follows

$$
\begin{aligned}
\operatorname{Pr}\left(c_{i, t}=h\right) \propto \exp \left[\left(\alpha^{h}-\alpha^{H}\right)\right. & +\left(\beta_{A, 1}^{h}-\beta_{A, 1}^{H}\right) \pi_{A, t-1}+\cdots+\left(\beta_{H, 1}^{h}-\beta_{H, 1}^{H}\right) \pi_{H, t-1} \\
& \left.+\left(\beta_{A, 2}^{h}-\beta_{A, 2}^{H}\right) \pi_{A, t-2}+\cdots+\left(\beta_{H, 2}^{h}-\beta_{H, 2}^{H}\right) \pi_{H, t-2}+\ldots\right] .
\end{aligned}
$$

The coefficients to be estimated for this model are the predispositions of alternatives $h=$ $A, B, \ldots, H^{-}$relative to that of alternative $H, \alpha^{h}-\alpha^{H}$, and the relative intensities of choice of alternative $h=A, B, \ldots, H^{-}$with respect to alternative $H, \beta_{k, l}^{h}-\beta_{k, l}^{H}$, given the payoffs of the different choices at different lags.

In this paper we estimate different versions of model (10), including those that are described by equations (1) and (2) in the main text (see below how these models are derived from (10)) and test whether behavioral simplifications often implicitly made in theoretical work are consistent with the experimental data. 


\section{F.1 Models with One Lag}

Consider the one-lag version of model (10) which, after normalizing the probabilities, gives (the subscripts for the single lag have been suppressed)

$$
\operatorname{Pr}\left(c_{i, t}=h\right)=\frac{\exp \left[\left(\alpha^{h}-\alpha^{H}\right)+\left(\beta_{A}^{h}-\beta_{A}^{H}\right) \pi_{A, t-1}+\cdots+\left(\beta_{H}^{h}-\beta_{H}^{H}\right) \pi_{H, t-1}\right]}{\sum_{k=A}^{H^{-}} \exp \left[\left(\alpha^{k}-\alpha^{H}\right)+\left(\beta_{A}^{k}-\beta_{A}^{H}\right) \pi_{A, t-1}+\cdots+\left(\beta_{H}^{k}-\beta_{H}^{H}\right) \pi_{H, t-1}\right]+1},
$$

for $h=A, B, \ldots, H^{-}$, with the remaining probability given by $\operatorname{Pr}\left(c_{i, t}=H\right)=1-\sum_{h=A}^{H^{-}} \operatorname{Pr}\left(c_{i, t}=h\right)$. We refer to model (11) as the General1 model.

\section{F.1.1 Binary Choice}

When there are only two alternatives, $A$ and $B$, the (binomial) logit model gives

$$
\operatorname{Pr}\left(c_{i, t}=A\right)=\frac{\exp \left[\left(\alpha^{A}-\alpha^{B}\right)+\left(\beta_{A}^{A}-\beta_{A}^{B}\right) \pi_{A, t-1}+\left(\beta_{B}^{A}-\beta_{B}^{B}\right) \pi_{B, t-1}\right]}{\exp \left[\left(\alpha^{A}-\alpha^{B}\right)+\left(\beta_{A}^{A}-\beta_{A}^{B}\right) \pi_{A, t-1}+\left(\beta_{B}^{A}-\beta_{B}^{B}\right) \pi_{B, t-1}\right]+1} .
$$

There are three (composite) coefficients to be estimated, which we will denote by

$$
\alpha=\alpha^{A}-\alpha^{B}, \beta_{A}=\beta_{A}^{A}-\beta_{A}^{B} \text { and }-\beta_{B}=\beta_{B}^{A}-\beta_{B}^{B} .
$$

In the case of binary choice, therefore, the most general one-lag model (General1) coincides with the PreAsym1 model (4), which we discussed in the main text.

The PreAsym 1 model is characterized by a predisposition effect, $\alpha$, and - potentially different - intensities of choices, $\beta_{A}$ and $\beta_{B}$. If we impose the restriction that the two intensities of choice are the same, $\beta_{A}=\beta_{B}=\beta$, we obtain the PreSym1 model, corresponding to

$$
\operatorname{Pr}\left(c_{i, t}=A\right)=\frac{\exp \left[\alpha+\beta\left(\pi_{A, t-1}-\pi_{B, t-1}\right)\right]}{\exp \left[\alpha+\beta\left(\pi_{A, t-1}-\pi_{B, t-1}\right)\right]+1}=\frac{\exp \left[\alpha+\beta \pi_{A, t-1}\right]}{\exp \left[\alpha+\beta \pi_{A, t-1}\right]+\exp \left[\beta \pi_{B, t-1}\right]},
$$

which only has the parameters $\alpha$ and $\beta$.

Finally, imposing the additional restriction $\alpha=0$ results in model (1):

$$
\operatorname{Pr}\left(c_{i, t}=A\right)=\frac{\exp \left[\beta \pi_{A, t-1}\right]}{\exp \left[\beta \pi_{A, t-1}\right]+\exp \left[\beta \pi_{B, t-1}\right]},
$$

which we refer to as the $\mathbf{S y m} \mathbf{1}$ model and has only one parameter (the intensity of choice $\beta$ ). 


\section{F.1.2 Choice between Three Alternatives}

When there are three alternatives, $A, B$ and $C$, the General1 model becomes

$$
\begin{aligned}
& \operatorname{Pr}\left(c_{i, t}=A\right)=\frac{\exp \left[\left(\alpha^{A}-\alpha^{C}\right)+\left(\beta_{A}^{A}-\beta_{A}^{C}\right) \pi_{A, t-1}+\left(\beta_{B}^{A}-\beta_{B}^{C}\right) \pi_{B, t-1}+\left(\beta_{C}^{A}-\beta_{C}^{C}\right) \pi_{C, t-1}\right]}{\exp \left[\left(\alpha^{A}-\alpha^{C}\right)+\sum_{h=A}^{C}\left(\beta_{h}^{A}-\beta_{h}^{C}\right) \pi_{h, t-1}\right]+\exp \left[\left(\alpha^{B}-\alpha^{C}\right)+\sum_{h=A}^{C}\left(\beta_{h}^{B}-\beta_{h}^{C}\right) \pi_{h, t-1}\right]+1} \\
& \operatorname{Pr}\left(c_{i, t}=B\right)=\frac{\exp \left[\left(\alpha^{B}-\alpha^{C}\right)+\left(\beta_{A}^{B}-\beta_{A}^{C}\right) \pi_{A, t-1}+\left(\beta_{B}^{B}-\beta_{B}^{C}\right) \pi_{B, t-1}+\left(\beta_{C}^{B}-\beta_{C}^{C}\right) \pi_{C, t-1}\right]}{\exp \left[\left(\alpha^{A}-\alpha^{C}\right)+\sum_{h=A}^{C}\left(\beta_{h}^{A}-\beta_{h}^{C}\right) \pi_{h, t-1}\right]+\exp \left[\left(\alpha^{B}-\alpha^{C}\right)+\sum_{h=A}^{C}\left(\beta_{h}^{B}-\beta_{h}^{C}\right) \pi_{h, t-1}\right]+1},
\end{aligned}
$$

with $\operatorname{Pr}\left(c_{i, t}=C\right)=1-\operatorname{Pr}\left(c_{i, t}=A\right)-\operatorname{Pr}\left(c_{i, t}=B\right)$. Eight parameters need to be estimated: two relative predispositions, $\alpha^{A}-\alpha^{C}$ and $\alpha^{B}-\alpha^{C}$, and six relative intensities of choice, $\beta_{k}^{A}-\beta_{k}^{C}$ and $\beta_{k}^{B}-\beta_{k}^{C}$ for $k=A, B, C$.

In order to obtain the PreAsym1 model we impose the following three restrictions

$$
\beta_{A}^{B}=\beta_{A}^{C}, \beta_{B}^{A}=\beta_{B}^{C} \text { and } \beta_{C}^{A}=\beta_{C}^{B} .
$$

These restrictions imply that the probabilities of choosing any two different alternatives respond in the same way to a change in the payoff associated to the third alternative. If these restrictions are not rejected and we denote

$$
\beta_{A}=\beta_{A}^{A}-\beta_{A}^{C}, \beta_{B}=\beta_{B}^{B}-\beta_{B}^{C}, \text { and }-\beta_{C}=\beta_{C}^{A}-\beta_{C}^{C},
$$

then model (14) simplifies to

$\operatorname{Pr}\left(c_{i, t}=h\right)=\frac{\exp \left[\alpha^{h}+\beta_{h} \pi_{h, t-1}\right]}{\exp \left[\alpha^{A}+\beta_{A} \pi_{A, t-1}\right]+\exp \left[\alpha^{B}+\beta_{B} \pi_{B, t-1}\right]+\exp \left[\alpha^{C}+\beta_{C} \pi_{C, t-1}\right]}, \quad h=A, B, C$.

This model has five parameters: two relative predisposition effects, $\alpha^{A}-\alpha^{C}$ and $\alpha^{B}-\alpha^{C}$, and three intensities of choice, $\beta_{A}, \beta_{B}$ and $\beta_{C}$.

The PreSym1 model can be obtained from the PreAsym1 model with two additional restrictions, $\beta_{B}=\beta_{A}$ and $\beta_{C}=\beta_{A}$ (using the notation from (16)). The resulting model is (denoting $\beta_{A}$ by $\beta$ ):

$$
\operatorname{Pr}\left(c_{i, t}=h\right)=\frac{\exp \left[\alpha^{h}+\beta \pi_{h, t-1}\right]}{\exp \left[\alpha^{A}+\beta \pi_{A, t-1}\right]+\exp \left[\alpha^{B}+\beta \pi_{B, t-1}\right]+\exp \left[\alpha^{C}+\beta \pi_{C, t-1}\right]}, \quad h=A, B, C .
$$

This model has three parameters: the two relative predisposition effects, $\alpha^{A}-\alpha^{C}$ and $\alpha^{B}-\alpha^{C}$, and the intensity of choice $\beta$.

Finally, imposing the restrictions $\alpha^{A}=\alpha^{C}$ and $\alpha^{B}=\alpha^{C}$ we end up with the Sym1 model which is

$$
\operatorname{Pr}\left(c_{i, t}=h\right)=\frac{\exp \left[\beta \pi_{h, t-1}\right]}{\exp \left[\beta \pi_{A, t-1}\right]+\exp \left[\beta \pi_{B, t-1}\right]+\exp \left[\beta \pi_{C, t-1}\right]} .
$$

This model has only one parameter, the intensity of choice, $\beta=\beta_{A}^{A}-\beta_{A}^{C}$. 


\section{F.2 Choice between Four Alternatives}

Finally, when the choice is between four alternatives $(A, B, C$ and $D)$ the General1 model becomes

$\operatorname{Pr}\left(c_{i, t}=A\right)=\exp \left[\left(\alpha^{A}-\alpha^{D}\right)+\left(\beta_{A}^{A}-\beta_{A}^{D}\right) \pi_{A, t-1}+\left(\beta_{B}^{A}-\beta_{B}^{D}\right) \pi_{B, t-1}+\left(\beta_{C}^{A}-\beta_{C}^{D}\right) \pi_{C, t-1}+\left(\beta_{D}^{A}-\beta_{D}^{D}\right) \pi_{D, t-1}\right] / Z$

$\operatorname{Pr}\left(c_{i, t}=B\right)=\exp \left[\left(\alpha^{B}-\alpha^{D}\right)+\left(\beta_{A}^{B}-\beta_{A}^{D}\right) \pi_{A, t-1}+\left(\beta_{B}^{B}-\beta_{B}^{D}\right) \pi_{B, t-1}+\left(\beta_{C}^{B}-\beta_{C}^{D}\right) \pi_{C, t-1}+\left(\beta_{D}^{B}-\beta_{D}^{D}\right) \pi_{D, t-1}\right] / Z$

$\operatorname{Pr}\left(c_{i, t}=C\right)=\exp \left[\left(\alpha^{C}-\alpha^{D}\right)+\left(\beta_{A}^{C}-\beta_{A}^{D}\right) \pi_{A, t-1}+\left(\beta_{B}^{C}-\beta_{B}^{D}\right) \pi_{B, t-1}+\left(\beta_{C}^{C}-\beta_{C}^{D}\right) \pi_{C, t-1}+\left(\beta_{D}^{C}-\beta_{D}^{D}\right) \pi_{D, t-1}\right] / Z$,

where $Z$ is the normalizing constant and $\operatorname{Pr}\left(c_{i, t}=D\right)=1-\operatorname{Pr}\left(c_{i, t}=A\right)-\operatorname{Pr}\left(c_{i, t}=B\right)-$ $\operatorname{Pr}\left(c_{i, t}=C\right)$. In this case, fifteen parameters need to be estimated: three relative predispositions, $\alpha^{A}-\alpha^{D}, \alpha^{B}-\alpha^{D}$ and $\alpha^{C}-\alpha^{D}$, and twelve relative intensities of choice: $\beta_{k}^{A}-\beta_{k}^{D}$, $\beta_{k}^{B}-\beta_{k}^{D}$ and $\beta_{k}^{C}-\beta_{k}^{D}$ for $k=A, B, C, D$.

Similar to the case with three choices we obtain the PreAsym1 model if we impose that the respective probabilities of choosing two different alternatives respond in a same way to a change in the payoffs of a third alternative. This gives the following eight restrictions

$$
\beta_{A}^{B}=\beta_{A}^{C}=\beta_{A}^{D}, \beta_{B}^{A}=\beta_{B}^{C}=\beta_{B}^{D}, \beta_{C}^{A}=\beta_{C}^{B}=\beta_{C}^{D} \text { and } \beta_{D}^{A}=\beta_{D}^{B}=\beta_{D}^{C}
$$

If these restrictions are not rejected and we introduce the following notation

$$
\beta_{A}=\beta_{A}^{A}-\beta_{A}^{D}, \beta_{B}=\beta_{B}^{B}-\beta_{B}^{D}, \beta_{C}=\beta_{C}^{C}-\beta_{C}^{D} \text { and }-\beta_{D}=\beta_{D}^{A}-\beta_{D}^{D}
$$

then we can write the PreAsym1 model as

$\operatorname{Pr}\left(c_{i, t}=h\right)=\frac{\exp \left[\alpha^{h}+\beta_{h} \pi_{h, t-1}\right]}{\exp \left[\alpha^{A}+\beta_{A} \pi_{A, t-1}\right]+\exp \left[\alpha^{B}+\beta_{B} \pi_{B, t-1}\right]+\exp \left[\alpha^{C}+\beta_{C} \pi_{C, t-1}\right]+\exp \left[\alpha^{D}+\beta_{D} \pi_{D, t-1}\right]}$,

for $h=A, B, C, D$. In this model seven parameters can be identified: three relative predisposition effects, $\alpha^{A}-\alpha^{D}, \alpha^{B}-\alpha^{D}$ and $\alpha^{C}-\alpha^{D}$, and four intensities of choice, $\beta_{A}, \beta_{B}, \beta_{C}$ and $\beta_{D}$.

The PreSym1 model is obtained from the PreAsym1 model with the three restrictions $\beta_{A}=\beta_{B}=\beta_{C}=\beta_{D}$ (using the notation from (17)). The resulting PreSym1 model (denoting the common intensity of choice by $\beta$ ) is

$\operatorname{Pr}\left(c_{i, t}=h\right)=\frac{\exp \left[\alpha^{h}+\beta \pi_{h, t-1}\right]}{\exp \left[\alpha^{A}+\beta \pi_{A, t-1}\right]+\exp \left[\alpha^{B}+\beta \pi_{B, t-1}\right]+\exp \left[\alpha^{C}+\beta \pi_{C, t-1}\right]+\exp \left[\alpha^{D}+\beta \pi_{D, t-1}\right]}$,

for $h=A, B, C, D$. This model has four parameters: three relative predisposition effects, $\alpha^{A}-\alpha^{D}, \alpha^{B}-\alpha^{D}$ and $\alpha^{C}-\alpha^{D}$, and the intensity of choice $\beta$.

Finally, the Sym1 model is a restricted version of the PreSym1 model with the three restrictions $\alpha^{A}=\alpha^{B}=\alpha^{C}=\alpha^{D}$. The resulting model is

$$
\operatorname{Pr}\left(c_{i, t}=A\right)=\frac{\exp \left[\beta \pi_{h, t-1}\right]}{\exp \left[\beta \pi_{A, t-1}\right]+\exp \left[\beta \pi_{B, t-1}\right]+\exp \left[\beta \pi_{C, t-1}\right]+\exp \left[\beta \pi_{D, t-1}\right]},
$$


for $h=A, B, C, D$ and only has the intensity of choice, $\beta$, as a parameter.

\section{F.3 Models with Several Lags}

For the blocks with two alternatives, $\mathrm{A}$ and $\mathrm{B}$, we also estimate the binary choice models with multiple lags. In the most general model the choice is affected by $L_{A}$ lags of performance for alternative $\mathrm{A}$ and $L_{B}$ lags of performance for alternative $\mathrm{B}$. This model gives (with alternative B as "reference option")

$$
\begin{aligned}
\operatorname{Pr}\left(c_{i, t}=A\right) \propto \exp \left[\left(\alpha^{A}-\alpha^{B}\right)\right. & +\left(\beta_{A, 1}^{A}-\beta_{A, 1}^{B}\right) \pi_{A, t-1}+\left(\beta_{B, 1}^{A}-\beta_{B, 1}^{B}\right) \pi_{B, t-1}+ \\
& +\left(\beta_{A, 2}^{A}-\beta_{A, 2}^{B}\right) \pi_{A, t-2}+\left(\beta_{B, 2}^{A}-\beta_{B, 2}^{B}\right) \pi_{B, t-2}+ \\
& \left.+\cdots+\left(\beta_{A, L_{A}}^{A}-\beta_{A, L_{A}}^{B}\right) \pi_{A, t-L_{A}}+\cdots+\left(\beta_{B, L_{B}}^{A}-\beta_{B, L_{B}}^{B}\right) \pi_{B, t-L_{B}}\right] .
\end{aligned}
$$

By introducing the notation $\alpha=\alpha_{A}-\alpha_{B}, \beta_{A, \ell}=\beta_{A, \ell}^{A}-\beta_{A, \ell}^{B}$ for $1 \leq \ell \leq L_{A}$ and $-\beta_{B, \ell}=$ $\beta_{B, \ell}^{A}-\beta_{B, \ell}^{B}$ for $1 \leq \ell \leq L_{B}$ the model can be rewritten as

$$
\operatorname{Pr}\left(c_{i, t}=A\right)=\frac{\exp \left[\alpha+\sum_{\ell=1}^{L_{A}} \beta_{A, \ell} \pi_{A, t-\ell}\right]}{\exp \left[\alpha+\sum_{\ell=1}^{L_{A}} \beta_{A, \ell} \pi_{A, t-\ell}\right]+\exp \left[\sum_{\ell=1}^{L_{B}} \beta_{B, \ell} \pi_{B, t-\ell}\right]} .
$$

In the main text we discuss the special case of $L_{A}=L_{B}=L$, when the model becomes (5), which we call PreAsymL. The model has $2 L+1$ parameters to be estimated. Note that the number of lags necessary to include can be decided using various tests. For instance, model PreSymL-1 is nested within PreSymL when the two restrictions $\beta_{A, L}=0$ and $\beta_{B, L}=0$ are imposed.

Imposing the $L$ restrictions $\beta_{A, \ell}=\beta_{B, \ell}$ for every $1 \leq \ell \leq L$, we obtain model PreSymL with $L+1$ parameters, where there is no asymmetry in responses to the performances of the two alternatives. With one additional restriction, $\alpha=0$, we obtain model SymL with $L$ parameters.

Finally, we might impose the following $L-2$ restrictions in model PreSymL

$$
\frac{\beta_{L}}{\beta_{L-1}}=\cdots=\frac{\beta_{\ell}}{\beta_{\ell-1}}=\cdots=\frac{\beta_{2}}{\beta_{1}}, \text { for all } 2 \leq \ell \leq L
$$

and denoting the ratio between subsequent intensities of choice as $\eta$ (and $\beta=\beta_{1}$ ) we get the following model

$$
\operatorname{Pr}\left(c_{i, t}=A\right)=\frac{\exp \left[\alpha+\beta\left(\pi_{A, t-1}+\eta \pi_{A, t-2}+\cdots+\eta^{L-1} \pi_{A, t-L}\right)\right]}{\exp \left[\alpha+\beta \sum_{\ell=1}^{L} \eta^{\ell-1} \pi_{A, t-\ell}\right]+\exp \left[\alpha+\beta \sum_{\ell=1}^{L} \eta^{\ell-1} \pi_{B, t-\ell}\right]} .
$$

This model (typically with $\alpha=0$ ) has been occasionally used in the literature on heterogeneous agent modelling, see footnote 33. The parameter $\eta$ is then referred to as the memory parameter. 


\section{F.4 Method of Maximisation of Likelihood}

In the paper we report the estimations of the general model (10) and its various restricted versions on aggregate data from different blocks of time series. To explain the estimation procedure we introduce the following general notation. Let $N$ be the number of observations for one block, $K$ the number of parameters to be estimated, $H$ the number of alternatives and $L$ the number of lags in the model.

Now we denote by $\boldsymbol{X}$ a $N \times(1+H L)$ matrix of exogenous regressors that is arranged in the following way. The first column of $\boldsymbol{X}$ is a vector of ones. The next $H$ columns (that is, column 2 until column $H+1$ ) correspond to the performances of the $H$ alternatives, lagged by one period. Columns $H+2$ until $2 H+1$ correspond to the performances of the $H$ alternatives, lagged by two periods, etc.

Subsequently, let $\boldsymbol{\beta}$ denote the vector of $K=(1+H L)(H-1)$ estimates, arranged as $\boldsymbol{\beta}=\left(\left(\boldsymbol{\beta}^{A}\right)^{\prime},\left(\boldsymbol{\beta}^{B}\right)^{\prime}, \ldots,\left(\boldsymbol{\beta}^{H^{-}}\right)^{\prime}\right)^{\prime}$, where the vector $\boldsymbol{\beta}^{h}$ (for $\left.h=A, B, \ldots, H^{-}\right)$collects the $1+H L$ coefficients that effect the proportionality coefficient of choosing alternative $h$ given in equation (10), that is it consists of $\alpha^{h}-\alpha^{H}, \beta_{A, 1}^{h}-\beta_{A, 1}^{H}$ up until $\beta_{H, L}^{h}-\beta_{H, L}^{H}$. The vector of estimates $\boldsymbol{\beta}$ therefore can be written as

$$
\begin{gathered}
\boldsymbol{\beta}=[\underbrace{\alpha^{A}-\alpha^{H}\left|\beta_{A, 1}^{A}-\beta_{A, 1}^{H}\right| \ldots\left|\beta_{H, 1}^{A}-\beta_{H, 1}^{H}\right| \ldots\left|\beta_{A, L}^{A}-\beta_{A, L}^{H}\right| \ldots \mid \beta_{H, L}^{A}-\beta_{H, L}^{H}}_{\left(\boldsymbol{\beta}^{\boldsymbol{A}}\right)^{\prime}} \mid \\
\underbrace{\left.\alpha^{B}-\alpha^{H}\left|\beta_{A, 1}^{B}-\beta_{A, 1}^{H}\right| \ldots\left|\beta_{H, 1}^{B}-\beta_{H, 1}^{H}\right| \ldots\left|\beta_{A, L}^{B}-\beta_{A, L}^{H}\right| \ldots\left|\beta_{H, L}^{B}-\beta_{H, L}^{H}\right| \ldots\right]^{\prime} .}_{\left(\boldsymbol{\beta}^{B}\right)^{\prime}}
\end{gathered}
$$

For example, in the General1 model with four alternatives, discussed in Section F.2, matrix $\boldsymbol{X}$ has five columns, and there are five scalars in each of $\boldsymbol{\beta}^{\boldsymbol{A}}, \boldsymbol{\beta}^{\boldsymbol{B}}$ and $\boldsymbol{\beta}^{\boldsymbol{C}}$, leading to a total of $K=15$ parameters to be estimated.

Using this notation the likelihood function can now be derived. Let $I_{i, t}^{h}$ be an index function of participant $i$ choosing alternative $h$ at time $t$, so that

$$
I_{i, t}^{h}= \begin{cases}1 & \text { if } c_{i, t}=h \\ 0 & \text { otherwise }\end{cases}
$$

For binary choice, for instance, we have $I_{i, t}^{B}=1-I_{i, t}^{A}$, and $I_{i, t}^{C}=I_{i, t}^{D}=0$. The likelihood function for an individual $i$ is given by

$$
\mathcal{L}_{i}(\boldsymbol{\beta})=\prod_{t=t_{0}}^{T} \operatorname{Pr}\left(c_{i, t}=A\right)^{I_{i, t}^{A}} \operatorname{Pr}\left(c_{i, t}=B\right)^{I_{i, t}^{B}} \operatorname{Pr}\left(c_{i, t}=C\right)^{I_{i, t}^{C}} \operatorname{Pr}\left(c_{i, t}=D\right)^{I_{i, t}^{D}}
$$

given that the model is estimated on data from period $t_{0}$ to $T$ (with $t_{0}=21$ and $T=40$ for the estimations presented in the main text). The product of all individual likelihood functions within the blocks with the same time series gives a likelihood function for the discrete choice 
model estimated on the aggregate data, which is

$$
\mathcal{L}(\boldsymbol{\beta})=\prod_{i} \mathcal{L}_{i}(\boldsymbol{\beta})
$$

For aggregate estimation we maximize function $\mathcal{L}(\boldsymbol{\beta})$ given by $(21)$. The sample size is $(T-$ $\left.t_{0}+1\right) n_{p}$, where the number of participants $n_{p}$ for a given block (or for two blocks with the same time series) is as reported in the last column of Table 1. Using (20) and (21) as well as (10) the log-likelihood function becomes

$$
\log \mathcal{L}(\boldsymbol{\beta})=\sum_{i} \log \mathcal{L}_{i}(\boldsymbol{\beta})=\sum_{i} \sum_{t=t_{0}}^{T}\left[\sum_{k=A}^{H^{-}} I_{i, t}^{k} \cdot \boldsymbol{X}_{\boldsymbol{t}} \boldsymbol{\beta}^{\boldsymbol{k}}-\log \left(1+\sum_{k=A}^{H^{-}} \exp \left(\boldsymbol{X}_{\boldsymbol{t}} \boldsymbol{\beta}^{\boldsymbol{k}}\right)\right)\right]
$$

where $\boldsymbol{X}_{\boldsymbol{t}}$ denotes the $\left(t-t_{0}+1\right)$ 'th row of matrix $\boldsymbol{X}$ (i.e., containing the performances from the previous time period).

For function (22) we compute the gradient vector whose $j$ 'th element $(j=1, \ldots, 1+H L)$ of the first part (corresponding to the derivative with respect to $\boldsymbol{\beta}^{\boldsymbol{A}}$ ) is

$$
\left[\frac{\partial \log \mathcal{L}(\boldsymbol{\beta})}{\partial \boldsymbol{\beta}^{\boldsymbol{A}}}\right]_{j}=\sum_{i} \sum_{t=t_{0}}^{T}\left(I_{i, t}^{A}-\frac{\exp \left(\boldsymbol{X}_{\boldsymbol{t}} \boldsymbol{\beta}^{\boldsymbol{A}}\right)}{1+\exp \left(\boldsymbol{X}_{\boldsymbol{t}} \boldsymbol{\beta}^{\boldsymbol{A}}\right)+\exp \left(\boldsymbol{X}_{\boldsymbol{t}} \boldsymbol{\beta}^{\boldsymbol{B}}\right)+\exp \left(\boldsymbol{X}_{\boldsymbol{t}} \boldsymbol{\beta}^{\boldsymbol{C}}\right)}\right)\left[\boldsymbol{X}_{\boldsymbol{t}}\right]_{j}
$$

where $\left[\boldsymbol{X}_{\boldsymbol{t}}\right]_{j}$ is the $j^{\prime}$ th element of $\boldsymbol{X}_{\boldsymbol{t}}$. Similar expressions describe the derivatives with respect to the components of vectors $\boldsymbol{\beta}^{\boldsymbol{B}}$ and $\boldsymbol{\beta}^{\boldsymbol{C}}$. Setting the gradient vector to zero we get a system of first-order conditions, which we solve numerically to find the estimates. ${ }^{42}$

The Hessian matrix for the log-likelihood function is given by

$$
\boldsymbol{H}=\left(\begin{array}{lll}
\frac{\partial^{2} \log \mathcal{L}(\boldsymbol{\beta})}{\partial \boldsymbol{\beta}^{\boldsymbol{A}} \partial\left(\boldsymbol{\beta}^{\boldsymbol{A}}\right)^{\prime}} & \frac{\partial^{2} \log \mathcal{L}(\boldsymbol{\beta})}{\partial \boldsymbol{\beta}^{\boldsymbol{A}} \partial\left(\boldsymbol{\beta}^{\boldsymbol{B}}\right)^{\prime}} & \frac{\partial^{2} \log \mathcal{L}(\boldsymbol{\beta})}{\partial \boldsymbol{\beta}^{\boldsymbol{A}} \partial\left(\boldsymbol{\beta}^{\boldsymbol{C}}\right)^{\prime}} \\
\frac{\partial^{2} \log \mathcal{L}(\boldsymbol{\beta})}{\partial \boldsymbol{\beta}^{\boldsymbol{B}} \partial\left(\boldsymbol{\beta}^{\boldsymbol{A}}\right)^{\prime}} & \frac{\partial^{2} \log \mathcal{L}(\boldsymbol{\beta})}{\partial \boldsymbol{\beta}^{\boldsymbol{B}} \partial\left(\boldsymbol{\beta}^{\boldsymbol{B}}\right)^{\prime}} & \frac{\partial^{2} \log \mathcal{L}(\boldsymbol{\beta})}{\partial \boldsymbol{\beta}^{\boldsymbol{B}} \partial\left(\boldsymbol{\beta}^{\boldsymbol{C}}\right)^{\prime}} \\
\frac{\partial^{2} \log \mathcal{L}(\boldsymbol{\beta})}{\partial \boldsymbol{\beta}^{\boldsymbol{C}} \partial\left(\boldsymbol{\beta}^{\boldsymbol{A}}\right)^{\prime}} & \frac{\partial^{2} \log \mathcal{L}(\boldsymbol{\beta})}{\partial \boldsymbol{\beta}^{\boldsymbol{B}} \partial\left(\boldsymbol{\beta}^{\boldsymbol{C}}\right)^{\prime}} & \frac{\partial^{2} \log \mathcal{L}(\boldsymbol{\beta})}{\partial \boldsymbol{\beta}^{\boldsymbol{C}} \partial\left(\boldsymbol{\beta}^{\boldsymbol{C}}\right)^{\prime}}
\end{array}\right)
$$

\footnotetext{
${ }^{42}$ We apply the optimization routine fminunc in MATLAB with the minus sign since we actually minimize $-\log \mathcal{L}_{i}(\boldsymbol{\beta})$. The code is available at request. The formula above is implemented in the program loglikelihood to compute the value of variable llf. Note that in the data file, the choices are 1 (for A), 2 (for B), 3 (for C) and 4 (for D).
} 
The diagonal blocks of this matrix are

$$
\begin{aligned}
& \frac{\partial^{2} \log \mathcal{L}(\boldsymbol{\beta})}{\partial \boldsymbol{\beta}^{\boldsymbol{A}} \partial\left(\boldsymbol{\beta}^{\boldsymbol{A}}\right)^{\prime}}=-\sum_{i} \sum_{t=t_{0}}^{T} \frac{\exp \left(\boldsymbol{X}_{\boldsymbol{t}} \boldsymbol{\beta}^{\boldsymbol{A}}\right)\left(1+\exp \left(\boldsymbol{X}_{\boldsymbol{t}} \boldsymbol{\beta}^{\boldsymbol{B}}\right)+\exp \left(\boldsymbol{X}_{\boldsymbol{t}} \boldsymbol{\beta}^{\boldsymbol{C}}\right)\right)}{\left(1+\exp \left(\boldsymbol{X}_{\boldsymbol{t}} \boldsymbol{\beta}^{\boldsymbol{A}}\right)+\exp \left(\boldsymbol{X}_{\boldsymbol{t}} \boldsymbol{\beta}^{\boldsymbol{B}}\right)+\exp \left(\boldsymbol{X}_{\boldsymbol{t}} \boldsymbol{\beta}^{\boldsymbol{C}}\right)\right)^{2}} \boldsymbol{X}_{\boldsymbol{t}}^{\prime} \boldsymbol{X}_{\boldsymbol{t}}, \\
& \frac{\partial^{2} \log \mathcal{L}(\boldsymbol{\beta})}{\partial \boldsymbol{\beta}^{\boldsymbol{B}} \partial\left(\boldsymbol{\beta}^{\boldsymbol{B}}\right)^{\prime}}=-\sum_{i} \sum_{t=t_{0}}^{T} \frac{\exp \left(\boldsymbol{X}_{\boldsymbol{t}} \boldsymbol{\beta}^{\boldsymbol{B}}\right)\left(1+\exp \left(\boldsymbol{X}_{\boldsymbol{t}} \boldsymbol{\beta}^{\boldsymbol{A}}\right)+\exp \left(\boldsymbol{X}_{\boldsymbol{t}} \boldsymbol{\beta}^{\boldsymbol{C}}\right)\right)}{\left(1+\exp \left(\boldsymbol{X}_{\boldsymbol{t}} \boldsymbol{\beta}^{\boldsymbol{A}}\right)+\exp \left(\boldsymbol{X}_{\boldsymbol{t}} \boldsymbol{\beta}^{\boldsymbol{B}}\right)+\exp \left(\boldsymbol{X}_{\boldsymbol{t}} \boldsymbol{\beta}^{\boldsymbol{C}}\right)\right)^{2}} \boldsymbol{X}_{\boldsymbol{t}}^{\prime} \boldsymbol{X}_{\boldsymbol{t}}, \\
& \frac{\partial^{2} \log \mathcal{L}(\boldsymbol{\beta})}{\partial \boldsymbol{\beta}^{\boldsymbol{C}} \partial\left(\boldsymbol{\beta}^{\boldsymbol{C}}\right)^{\prime}}=-\sum_{i} \sum_{t=t_{0}}^{T} \frac{\exp \left(\boldsymbol{X}_{\boldsymbol{t}} \boldsymbol{\beta}^{\boldsymbol{C}}\right)\left(1+\exp \left(\boldsymbol{X}_{\boldsymbol{t}} \boldsymbol{\beta}^{\boldsymbol{A}}\right)+\exp \left(\boldsymbol{X}_{\boldsymbol{t}} \boldsymbol{\beta}^{\boldsymbol{B}}\right)\right)}{\left(1+\exp \left(\boldsymbol{X}_{\boldsymbol{t}} \boldsymbol{\beta}^{\boldsymbol{A}}\right)+\exp \left(\boldsymbol{X}_{\boldsymbol{t}} \boldsymbol{\beta}^{\boldsymbol{B}}\right)+\exp \left(\boldsymbol{X}_{\boldsymbol{t}} \boldsymbol{\beta}^{\boldsymbol{C}}\right)\right)^{2}} \boldsymbol{X}_{\boldsymbol{t}}^{\prime} \boldsymbol{X}_{\boldsymbol{t}},
\end{aligned}
$$

with the off-diagonal blocks given by

$$
\begin{aligned}
& \frac{\partial^{2} \log \mathcal{L}(\boldsymbol{\beta})}{\partial \boldsymbol{\beta}^{\boldsymbol{A}} \partial\left(\boldsymbol{\beta}^{\boldsymbol{B}}\right)^{\prime}}=\sum_{i} \sum_{t=t_{0}}^{T} \frac{\exp \left(\boldsymbol{X}_{\boldsymbol{t}} \boldsymbol{\beta}^{\boldsymbol{A}}\right) \exp \left(\boldsymbol{X}_{\boldsymbol{t}} \boldsymbol{\beta}^{\boldsymbol{B}}\right)}{\left(1+\exp \left(\boldsymbol{X}_{\boldsymbol{t}} \boldsymbol{\beta}^{\boldsymbol{A}}\right)+\exp \left(\boldsymbol{X}_{\boldsymbol{t}} \boldsymbol{\beta}^{\boldsymbol{B}}\right)+\exp \left(\boldsymbol{X}_{\boldsymbol{t}} \boldsymbol{\beta}^{\boldsymbol{C}}\right)\right)^{2}} \boldsymbol{X}_{\boldsymbol{t}}^{\prime} \boldsymbol{X}_{\boldsymbol{t}}, \\
& \frac{\partial^{2} \log \mathcal{L}(\boldsymbol{\beta})}{\partial \boldsymbol{\beta}^{\boldsymbol{A}} \partial\left(\boldsymbol{\beta}^{\boldsymbol{C}}\right)^{\prime}}=\sum_{i} \sum_{t=t_{0}}^{T} \frac{\exp \left(\boldsymbol{X}_{\boldsymbol{t}} \boldsymbol{\beta}^{\boldsymbol{A}}\right) \exp \left(\boldsymbol{X}_{\boldsymbol{t}} \boldsymbol{\beta}^{\boldsymbol{C}}\right)}{\left(1+\exp \left(\boldsymbol{X}_{\boldsymbol{t}} \boldsymbol{\beta}^{\boldsymbol{A}}\right)+\exp \left(\boldsymbol{X}_{\boldsymbol{t}} \boldsymbol{\beta}^{\boldsymbol{B}}\right)+\exp \left(\boldsymbol{X}_{\boldsymbol{t}} \boldsymbol{\beta}^{\boldsymbol{C}}\right)\right)^{2}} \boldsymbol{X}_{\boldsymbol{t}}^{\prime} \boldsymbol{X}_{\boldsymbol{t}}, \\
& \frac{\partial^{2} \log \mathcal{L}(\boldsymbol{\beta})}{\partial \boldsymbol{\beta}^{\boldsymbol{B} \partial\left(\boldsymbol{\beta}^{\boldsymbol{C}}\right)^{\prime}}}=\sum_{i} \sum_{t=t_{0}}^{T} \frac{\exp \left(\boldsymbol{X}_{\boldsymbol{t}} \boldsymbol{\beta}^{\boldsymbol{B}}\right) \exp \left(\boldsymbol{X}_{\boldsymbol{t}} \boldsymbol{\beta}^{\boldsymbol{C}}\right)}{\left(1+\exp \left(\boldsymbol{X}_{\boldsymbol{t}} \boldsymbol{\beta}^{\boldsymbol{A}}\right)+\exp \left(\boldsymbol{X}_{\boldsymbol{t}} \boldsymbol{\beta}^{\boldsymbol{B}}\right)+\exp \left(\boldsymbol{X}_{\boldsymbol{t}} \boldsymbol{\beta}^{\boldsymbol{C}}\right)\right)^{2}} \boldsymbol{X}_{\boldsymbol{t}}^{\prime} \boldsymbol{X}_{\boldsymbol{t}} .
\end{aligned}
$$

We numerically compute matrix $\boldsymbol{H}$ for the estimated $\hat{\boldsymbol{\beta}}$ and then compute the standard errors of the estimates as the square roots of the diagonal elements of the inverse of this matrix.

\section{Goodness-of-Fit}

To evaluate the goodness of fit of a particular model we compute the McFadden $\mathrm{R}^{2}$ (also known as likelihood ratio index) as follows

$$
1-\frac{\log \mathcal{L}(\hat{\boldsymbol{\beta}})}{\log \mathcal{L}(0)},
$$

where $\log \mathcal{L}(0)$ denotes the resulting $\log$-likelihood when only the constant term is estimated. ${ }^{43}$

\section{Model Comparison}

To compare different models we use several criteria. First, we report and compare the loglikelihood, $\log \mathcal{L}(\hat{\boldsymbol{\beta}})$, as well as the values for two information criteria penalizing for additional parameters. The first of those, the Akaike Information Criterion, is given by

$$
\mathrm{AIC}=2 k-2 \log \mathcal{L}(\hat{\boldsymbol{\beta}})
$$

\footnotetext{
${ }^{43}$ This measure cannot be larger than one, and it is never negative (except for model Sym1, where the constant term is absent). Its interpretation as the percentage explained by the model with respect to the most elementary model is not always useful, though the higher its value, the better.
} 
where $k$ corresponds to the number of parameters. The second criterion, the Bayesian Information Criterion, is computed as

$$
\mathrm{BIC}=k \log (N)-2 \log \mathcal{L}(\hat{\boldsymbol{\beta}}) .
$$

Minimizing each of these measures over different models can be used as a criterion for model selection. 